LABORATOIRE D'INFORMATIQUE, De Robotique et de Microélectronique De MontPellier

Unité Mixte CNRS - Université Montpellier II C 55060

RAPPORT DE RECHERCHE

\title{
Implementing Statically Typed Object-Oriented Programming Languages
}

\section{Roland Ducournau}

5 novembre 2002, révisé January 9, 2003 R.R.LIRMM 2002-174 
2 . Roland Ducournau 


\title{
Implementing Statically Typed Object-Oriented Programming Languages
}

\author{
ROLAND DUCOURNAU \\ LIRMM - CNRS et Université Montpellier II, France
}

\begin{abstract}
In statically typed object-oriented languages, message sending, or late binding, is implemented with tables often called virtual functions tables (VFT). Those tables reduce method calls to function calls, with a small fixed number of extra indirections. The overhead is more or less important, whether inheritance and subtyping are single or multiple.

In this paper, we survey the various implementation schemes available in separate compilation, in the three cases of single inheritance, multiple inheritance, and single inheritance but multiple subtyping, e.g. JAVA. Many works have been done recently in the framework of global compilation, mostly for dynamically typed languages but also applied to the EIFFEL language in the SMALL EIFFEL compiler. We examine how global compilation can improve the implementation and propose to use global techniques - coloring and type analysis - in a separate compilation framework.

Categories and Subject Descriptors: D.3.2 [Programming languages]: Language classifications_object-oriented languages; C++; Java; Eiffel; ThetA; D.3.4 [Programming languages]: Processors-compilers

General Terms: Languages, Measurement, Performance

Additional Key Words and Phrases: casting, coloring, contravariance, covariance, genericity, late binding, method dispatch, static typing, type analysis, separate compilation, virtual function tables
\end{abstract}

\section{INTRODUCTION}

In separate compilation of statically typed object-oriented languages, method calls - often named message sending or late binding - are generally implemented with tables called virtual function tables in the $\mathrm{C}++$ jargon. Method calls are then reduced to function calls with a small fixed number of extra indirections. An object is laid out as an attribute table, with a header pointing to the class table and some added information, e.g. for garbage collection. The cost of such an implementation depends on inheritance: with single inheritance, the overhead is reasonably small, but it is larger in the case of multiple inheritance. This paper aims to describe the various schemes generally used together with some alternatives, and to evaluate and compare them.

\subsection{Object-oriented mechanisms}

First, we will exclude from our examination all non $\mathrm{OO}$ features - e.g. non virtual methods in $\mathrm{C}++$, or static variables and functions in $\mathrm{C}++$ and JAVA-whose implementation is the same as in non OO languages. Two primary mechanisms are at the focus of OO implementation: read and write accesses to attributes and message sending, i.e. selection (also called dispatch) and call of the method corresponding to the receiver's dynamic type. A number of secondary mechanisms must also be considered. They are more or less explicit in the language design, but are all necessary and not so simple as one could expect:

-inclusion polymorphism - i.e. the fact that an entity of a given static type may be bound to a value of a dynamic subtype - may need special attention as an object reference, e.g. self $f^{1}$, may depends on its static type;

-attributes and methods overriding may be type invariant or not and, in the latter case, it can be type safe or unsafe: this is known as the covariance-contravariance problem;

\footnotetext{
${ }^{1}$ Self is the reserved word used in Smalltalk to name the message receiver: it corresponds to this in $\mathrm{C}++$ and JAVA and current in EIfFEL. An occurrence of self in a method of a given class has this class as its static type.
} 
— dynamic type checking is a need for constructs like downcast or typecase, as for unsafe overriding;

- class attributes, shared by all instances of a class, but reachable from the instance dynamic type in case of overriding, unlike static variables in $\mathrm{C}++$ and JAVA;

- call to super, a way for the overriding method to call the overridden one;

-null value, for uninitialized variables and attributes;

- parameterized classes, in a bounded genericity framework.

Only method selection on a single receiver will be considered here: multiple selection, as it is realized in CLOS [Steele 1990] and theorized by Castagna [1997], can use techniques connected to single selection, but the combinatory in the cartesian product of types may be explosive.

We shall no more consider the various mechanisms which, under various names as encapsulation, protection, visibility or export, rule the access rights of some entity by the other ones: they actually are only access rights to existing implementation. Anyway, an exception will be done for SMALLTALK encapsulation which reserves for self all accesses to attributes [Goldberg and Robson 1983]: this will reveal of interest for implementation.

Primitive types issue will be also evaded, particularly concerning the question of calling a method on a receiver statically typed by the universal type, common super-type of primitive types and object classes. Such a universal type, any in EIfFEL, does not exist neither in C++, nor in JAVA. We consider also that a value is either an immediate value of a primitive type, or the address of an object instance of some class. Thus we exclude the fact that an attribute value could be the object itself, as in $\mathrm{C}++$ or in EIFFEL with the keyword expanded: indeed, objects as immediate values forbid polymorphism for attributes and are incompatible with a notion of object identity ${ }^{2}$.

As for types and subtyping, we shall keep a common point of view: type safety is assumed but we will also consider the effect of a covariant policy on implementation [Castagna 1995; Meyer 1997; Ducournau 2002b]. Static type checking, at compile-time, is out of the scope of this paper: only dynamic type checking will be examined.

\subsection{Evaluating efficiency}

There are two basic criteria for efficiency: time and space. Time efficiency can be judged on average but the ideal thing is constant time mechanisms. Space efficiency is evaluated by the complete memory needed for run-time. Space and time efficiencies vary in opposite directions: a single criterion is impossible and a compromise is always needed. Eventually, run-time efficiency is the main goal but compile-time efficiency must not be forgotten: attention should be payed to NP-hard optimizations.

1.2.1 Space efficiency. Three kinds of memory resources must be considered. The dynamic part consists of the objects themselves, implemented as an attribute table, with one or more pointers to class tables: garbage collection must be envisaged for this part. The static part consists of the data structures associated to classes, which are read only and can be allocated in the code area, together with the code itself, where a given mechanism is handled by some number of instructions.

A static memory analysis has been done in the framework of global techniques which need to compact large tables [Driesen et al. 1995; Ducournau 1997], but it has not been done for separate compilation and static typing. As for dynamic memory, commonly used techniques may have a large overhead. First, some langages like JAVA may implement some mechanism through a constant dynamic overhead [Bacon et al. 2002]. Second, dynamic space may be sacrified for time efficiency, by putting part of static tables in dynamic tables: therefore dynamic overhead may need special optimizations [Eckel and Gil 2000].

1.2.2 Time efficiency and processor architecture. With classic processors, a measure of the time efficiency of a mechanism is the number of machine instructions needed for it. Modern processors

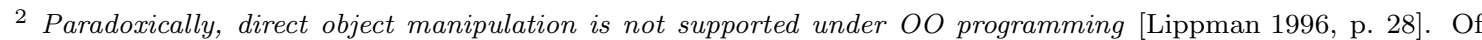
course, this is not paradoxical: essence of object-orientation is that the object manipulates itself.
} 
make this measure obsolete since they have a pipe-line architecture together with capabilities for parallelism (super-scalar processors) and branch prediction. In counterpart, memory access and unpredicted branch cause a multi-cycle latency. Thus the time spent for one instruction is no more one cycle. Moreover, composing two mechanisms may follow a law of the maximum instead of the sum. The instruction number remains only a space measure.

Implementing method calls with direct access in method tables has been for long considered as optimal: the effective overhead w.r.t. function calls seemed unavoidable. However, branching prediction of modern processors seems to have better performance with the technique, known as inline cache or type prediction, which consists in comparing the receiver's effective type with an expected type, the method of which is statically known. Such a test, statistically well predicted for whole programs, makes this technique very efficient [Driesen et al. 1995; Zendra et al. 1997]. Table-based techniques could be considered out of date. Nevertheless, two arguments are opposite to this thesis. Branching prediction favorizes type prediction as the former is restricted to conditional branching: but it could be extended, in future processors, to indirect branching, putting both techniques on an equal step [Driesen 1999]. Moreover, type prediction is not adapted to separate compilation.

We base our evaluation of time efficiency on an intuitive pseudo-code, mostly borrowed to [Driesen and Hölzle 1995; Driesen et al. 1995; Driesen 1999]. Each code sequence will be measured by an estimate of the number of cycles, parameterized by memory latency $-L$ whose value is 2 or 3 - and branching latency $-B$ whose value may run from 3 to 15 . More details can be found in the works by K. Driesen.

\subsection{Notations and conventions}

Uppercase letters will denote classes or types, according to the context. The class specialization relationship is denoted by $\prec_{\mathrm{d}}: B \prec_{\mathrm{d}} A$ means that $B$ is a direct subclass of $A$. One assumes that $\prec_{\mathrm{d}}$ has no transitive edges and its transitive (resp. and reflexive) closure is denoted by $\prec$ (resp. $\preceq)$ : the latter is a partial order. Subtyping is noted $<$ :, this is a preorder. We consider that classes are types and that class specialization is subtyping (i.e. $\preceq$ is a subset of $<$ :): even if type theory distinguishes both relationships, this is a common simplification in most languages. $\tau_{s}$ and $\tau_{d}$, where $\tau_{d}<: \tau_{s}$, denote respectively the static and dynamic types of an entity: the static type is an annotation in the program text, whereas the dynamic type is the class instanciating the value currently bound to the entity. At last, a root is a class (resp. type) without superclass (resp. supertype). As for properties, i.e. methods and attributes, we adopt the following vocabulary: a class has or knows a property if the property is defined in the class or in one of its super-classes. A class introduces a property when the property is defined in the class, not in its superclasses. We assume that all ambiguities caused by static overloading (à la $\mathrm{C}++$ or JAVA) have been solved by renaming [Meyer 2001; Ducournau 2002b]. Thus, the notation for a method (e.g. $m$ ) will denote both the method name and the generic property, invariant by inheritance and overriding, that this name unambiguously denotes ${ }^{3}$. Moreover, we are not concerned here by the inheritance problem itself, especially when multiple - i.e. which method is inherited by some dynamic type? - but only by an efficient way of calling the appropriate method, whatever it is. Therefore, we will use the terms attribute and method to denote generic properties and, in some rare cases, we will qualify them with definition to denote the occurrence of the generic property in some class.

\subsection{Context and plan}

This paper aims to survey implementation techniques, whether they are effectively implemented in some language, described in the literature, or merely imagined as a point in the state space of possible implementations. A difficulty arises as most effective implementations are not described in the literature, either because they are not assumed to be novel, or for confidentiality reasons, because they are. In the latter case, they are often covered by US patents. Conversely, many tech-

\footnotetext{
3 This notion of generic property is missing in most languages: the term was coined on the model of Clos generic function and it is an equivalent of EIFFEL feature. In the literature on method dispatch, the SMALLTALK term of method selector is often used, whereas Zibin and Gil [2002] use method family.
} 
Table I. Four kinds of languages

\begin{tabular}{c|cc|cc}
\hline & \multicolumn{4}{|c}{ subtyping } \\
inheritance & single & \multicolumn{2}{c}{ multiple } \\
\hline single & Simula, C++ (si) & SST & MST & JAVA, ThetA, Eiffel\#, C\# \\
\hline arborescent & - & AI & C ++ (non virtual) \\
\hline multiple & - & MI & C ++ (virtual), EiffEL \\
\hline
\end{tabular}

niques are theoretically described in the literature, without any known implementation. Language specifications could help to determine if a technique is adapted to some language, but currently, complete specifications are not always implemented. Thus, the schemes that we describe are more likely than real, but principles should not be too far. Moreover, it cannot be excluded that some techniques described here are novel, though it is not the paper goal.

Language implementation depends also on the way a program runs. Is it interpreted or compiled? Is compilation separate or global? Is linking static or dynamic? Is loading incremental? Our main objective is separate compilation, as it is common in most of the considered languages, but we will examine the effect of this choice on the possible techniques.

Structure of the paper. One can classify statically typed languages by their inheritance and subtyping relationships, according to whether they are single or multiple. Moreover, when inheritance is multiple, it may be arborescent, i.e. the superclasses of every class form a tree. Each of the four resulting categories uses different techniques (Table I). The next two sections present the standard implementation principles in the two extreme cases: single subtyping (SST) then multiple inheritance (MI). Various notions are precisely introduced on the way. In section 4, we examine some alternatives for multiple inheritance, among which $\mathrm{C}++$ "non virtual" implementation (NVI) for arborescent inheritance (AI). The next section describes the median case of single inheritance (SI) but multiple subtyping (MST), illustrated by JAVA and DotNet languages. Some applications to multiple inheritance and the special case of mixins are examined. Section 6 presents some complementary mechanisms not treated up to there: genericity, type variant overriding, class attributes. Whereas the previous sections consider the case of separate compilation, section 7 proposes a short survey of the techniques used in global compilation and dynamic typing. Two global techniques, coloring and type analysis, are sounded as they seem good candidates for use at link-time in a separate compilation framework. The article ends with a conclusion and some perspectives. Appendix presents space statistics on common benchmarks and the pseudo-code for all the presented techniques.

\section{SINGLE INHERITANCE AND SUBTYPING (SST)}

\subsection{Principle}

With single inheritance and subtyping, types may be identified with classes. Each class has at most one superclass. Thus the subclass tables are simply obtained by adding newly introduced methods and attributes at the end of the superclass tables (Figure 1). Two invariants characterize this implementation:

INVARIANT 2.1.1. A reference-method parameter, local variable, attribute or returned valueon an object is invariant w.r.t. the reference's static type.

INVARIANT 2.1.2. Each attribute or method $p$ has a unique offset, noted $\delta_{p}$, unambiguous and invariant by inheritance and w.r.t. the receiver's static type.

Thus, standard SST implementation is characterized by an absolute invariance w.r.t. static types. This enhances the basic semantics of object orientation, which states that dynamic type is the object's essence and that static types are pure contingency. Moreover, it realizes the ideal type erasure of type theory.

Method calls are then compiled by a sequence of three instructions:

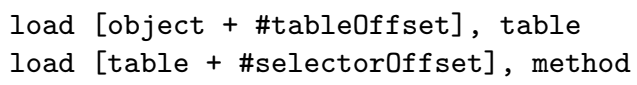




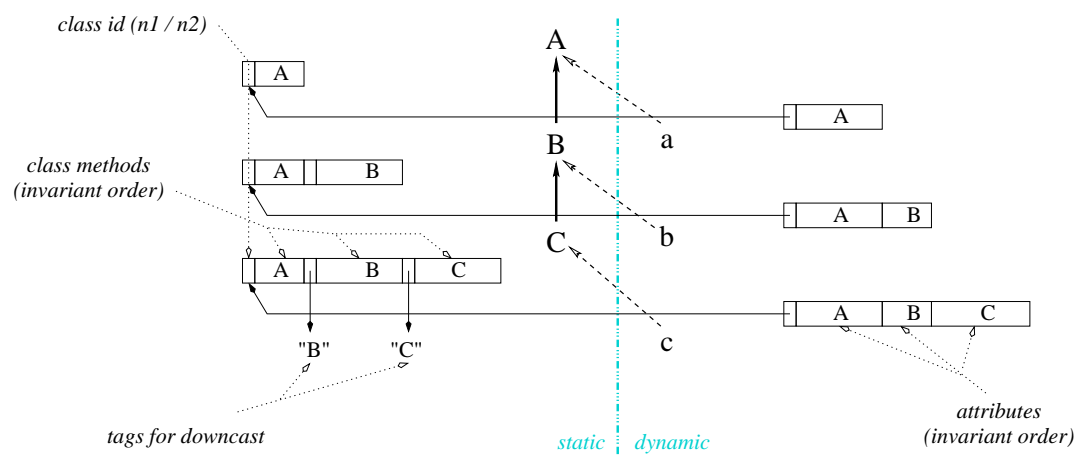

Fig. 1. Object layout and method tables in single subtyping: 3 classes $A, B$ et $C$ with their respective instances, $a, b$ and $c$.

call method

and attribute accesses are as immediate as for a record field:

load [object + \#attribute0ffset], attribute

Notice that attribute invariance is due to the fact that attributes have always fixed size values: when an attribute type has variable size values, the attribute is a pointer to those values.

Computing the tables, i.e. method and attribute offsets, is a special algorithmic case of the coloring heuristics (see section 7.4). Single inheritance and static typing, which avoids definition overloading ${ }^{4}$, ensure a sound and optimal result: no offset conflict can occur between inherited properties and there is no need to check that the next free offset is really free.

Though pure SST languages are not common, this implementation is the basis of most implementation, as well JAVA without interfaces (see section 5) as $\mathrm{C}++$ when restricted to single and "non virtual" inheritance (see section 4.1).

\subsection{Casting}

2.2.1 Principle. The word cast is commonly used to term various mechanisms close to type coercion, from a source type to a target type. Among the various interpretations, two are of interest for object-orientation, as they concern the relationship between static and dynamic types: source and target types are then related by subtyping.

Upcast is often called implicit because it needs no particular syntax. It simply consists in a polymorphic assignment (or parameter passing) $x:=y$, when the static types of $x$ and $y$ are respectively $X$ and $Y$ and when $Y$ is a proper subtype of $X(Y<: X)$. Such a mechanism should have no name as it is conceptually empty - this is pure inclusion polymorphism - but its implementation may be non trivial.

Downcast amounts to assume that an entity of a given static type $\tau_{s}=X$ is actually an instance of a subtype $Y$, i.e. $\tau_{d}<: Y$ and, usually, $Y<: X$. This is a type unsafe assumption, which cannot be statically checked (without loss of generality): it thus needs a dynamic type check which may signal an exception if it turns out that the assumption was false. Downcast is realized by special syntactic constructs as dynamic_cast in $\mathrm{C}++$, parenthesized syntax in JAVA (not to use in C++), typecase in TheTA [Liskov et al. 1995] or assignment attempts in Eiffel [Meyer 1992; 1997]. Downcast uses are often justified by the fact that covariant models are implemented in type safe, i.e. contravariant or invariant, languages (see section 6.1). They are also common in JAvA because of its lack of genericity.

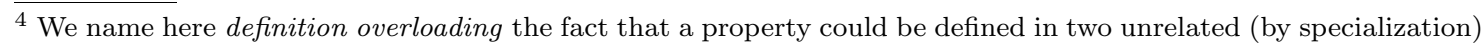
classes, without being defined in any common superclass: with static typing the two occurrences are no more related than with static overloading but this is no more the case with dynamic typing, e.g. in SMALLTALK.
} 
Besides those two opposite directions, casting may also be static or dynamic ${ }^{5}$, according to whether the target type is statically known or not. Explicit syntactic constructs are always static casts: the target type is a constant of the construct. However, we will see that some mechanisms may need dynamic casts, which impose that the target type is reachable from the considered object.

2.2.2 Casting in single subtyping. As references to objects don't depend on static types, upcast has no more reality in the implementation than as a concept.

Downcast. Due to reference invariance, this amounts to dynamic type checking. There are two classic ways to implement type checking with SST. The first one consists in assigning an offset to each class in the method tables of its subclasses: the corresponding entry of the tables must contain the class identifier. An object is an instance of a class $C$ iff the object's method table, noted $t a b_{\tau_{d}}$, contains at the offset $\delta_{C}$ the identifier $i d_{C}$ :

$$
\tau_{d} \preceq C \Leftrightarrow t a b_{\tau_{d}}\left[\delta_{C}\right]=i d_{C}
$$

Class offsets are ruled by the same Invariant 2.1.2 as methods and attributes. This first technique is simple and works in separate compilation: it is time efficient, but not space optimal. This is again a special case of coloring (see section 7.4), described by Cohen [1991] and reused by Queinnec [1997].

The second technique is optimal but has a twofold drawback: it does not generalize to multiple inheritance, at least in constant time, and it is not incremental, thus incompatible with dynamic loading. It is a double class numbering, noted $n_{1}$ and $n_{2}: n_{1}$ is a preorder depth-first numbering of the inheritance tree and $n_{2}$ is defined by $n_{2}(C)=\max _{D \preceq C}\left(n_{1}(D)\right)$. Then:

$$
\tau_{d} \prec C \Leftrightarrow n_{1}(C)<n_{1}\left(\tau_{d}\right) \leq n_{2}(C)
$$

This technique, due to Schubert et al. [1983], is often called Schubert's numbering or relative numbering. Only two short integers are needed and the first one $\left(n_{1}\right)$ can serve as class identifier. For the test $(2), n_{1}\left(\tau_{d}\right)$ is dynamic, whereas $n_{1}(C)$ and $n_{2}(C)$ are static when the cast is static: they may be compiled as constants. Both techniques have the same time efficiency ( $2 L+2$ cycles). The latter has a better memory cost in the static tables (worst-case linear instead of quadratic) but the former has a more compact code (4 instructions instead of 6). Type checking remains an active topic of research, even in single subtyping [Raynaud and Thierry 2001; Zibin and Gil 2001], but no other technique has such a power of simplicity.

\subsection{Evaluation}

Time efficiency is optimal as everything is done with at most a single indirection in a table. Even downcasts are constant-time. Dynamic space efficiency is also optimal: object layout is akin to record layout, with the only overhead of a single pointer to class method table. Method tables depend only on object dynamic types. As a whole, they occupy a place equal to the number of valid class-method pairs, which is the optimal compactness of the class-method tables used by constant-time techniques in dynamic typing, multiple inheritance and global compilation (see section 7). Let $M_{C}$ denote the number of methods known (defined or inherited) by a class $C$ : then the method tables have $\Sigma_{C} M_{C}$ entries.

This optimal implementation of SST is the reference which serves to measure the overhead of multiple inheritance or subtyping, for both time and space efficiency. On this basis, intraprocedural type analysis may detect monomorphic cases, when $\tau_{d}$ is known at compile-time: method calls are then static function calls, without table accesses, and inlining is possible.

\section{MULTIPLE INHERITANCE (MI)}

Multiple inheritance complicates the implementation to a considerable extent, as [Ellis and Stroustrup 1990, chapter 10] and [Lippman 1996] demonstrate it for $\mathrm{C}++. \mathrm{C}++$ is all the more complicated be-

\footnotetext{
5 Those terms are unrelated with the $\mathrm{C}++$ keywords static_cast and dynamic_cast which are both static (see section 4.1).
}

RR LIRMM 2002-174 


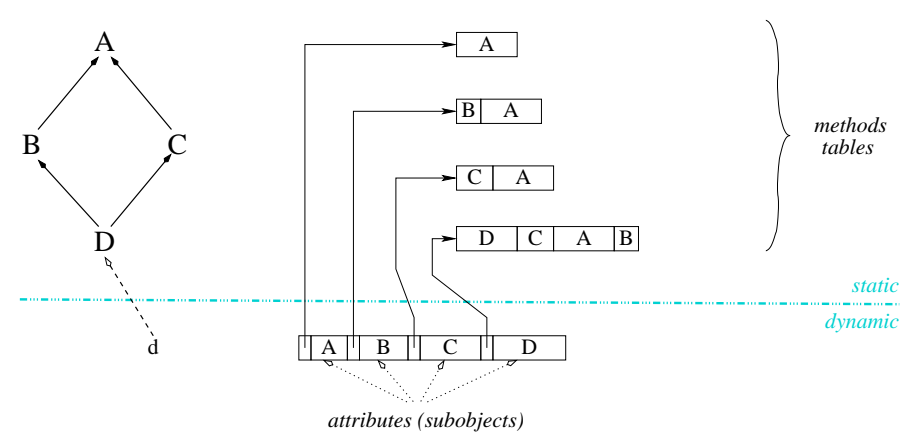

Fig. 2. Object layout and method tables with multiple inheritance

cause it offers some unsound features which are aimed to reduce MI overhead. The keyword virtual, when used to annotate superclasses, is the way to obtain a sound MI semantics whereas what we will term non virtual multiple inheritance (NVI), when the keyword is not used, offers a cheaper implementation but degraded semantics, sound only for arborescent inheritance (AI) (see section 4.1). Therefore, in this section, we consider that, in $\mathrm{C}++$, we would have to use virtual to annotate every superclass. Those introductory precautions are not necessary for an OO langage of sound constitution as EIFFEL [Meyer 1992; 1997].

\subsection{Principle}

In separate compilation and MI, there is no way to assign a minimal and invariant offset to each method and attribute (Invariant 2.1.2) without causing future conflicts. Let $B$ and $C$ two unrelated classes occupying the same offsets: then, it will always be possible to define a common subclass to those two classes, say $D$ (Figure 2). Two attributes (or methods) respectively of $B$ and $C$, both present in $D$, will conflict for the same offset. Giving up Invariant 2.1.2 leads to do the same for Invariant 2.1.1, if one wants that method calls take only one indirection in a table. We will see further how to keep reference invariance, by giving up constant-time mechanisms (see section 5.4) or separate compilation (see section 7.4).

3.1.1 Object layout and method tables. Offset invariance is thus relaxed, in two different ways for attributes and methods.

INVARIANT 3.1.1. Each attribute has an offset, noted $\delta_{a}$, unambiguous and invariant, in the context only of the static type which introduces the attribute.

INVARIANT 3.1.2. Each method has an offset, noted $\delta_{m}^{\tau_{s}}$, unambiguous and invariant in the context of every static type which knows the method.

The object consists of subobjects, one for each superclass of its class (i.e. for each static type supertype of its dynamic type) and each subobject is equipped with its own method tables (Figure 2). Reference invariance is then replaced by:

InVARIANT 3.1.3. Any entity whose static type is $T$ is bound to the subobject corresponding to T. Subobjects of different static types are distinct.

A subobject consists only of the attributes introduced by the corresponding static type, whereas the method table contains all the methods known by it, with values (addresses) corresponding to methods inherited by the dynamic type. Thus, two proper instances of different classes will not share any method table of their common superclasses: thoses tables are isomorphic but don't contain the same addresses (Table II). For a given static type, method offsets don't matter, but it is reasonable to group methods by introduction classes, as in the figures: this may offer some local invariance (in case of SI) but this organization has no effect on efficiency.

3.1.2 Method call. Invariant 3.1.3 imposes to recompute the value of self for each method call where the receiver's static type $\tau_{s}$ is different from the class $W$, superclass of $\tau_{d}$, which defined 
Table II. Method tables for Figure 2 example, w.r.t. static and dynamic types. For a same static type (column), the tables are isomorphic but differ by their contents (method addresses), whereas, for a same dynamic type (row), isomorphic pieces contain the same addresses but different shifts.

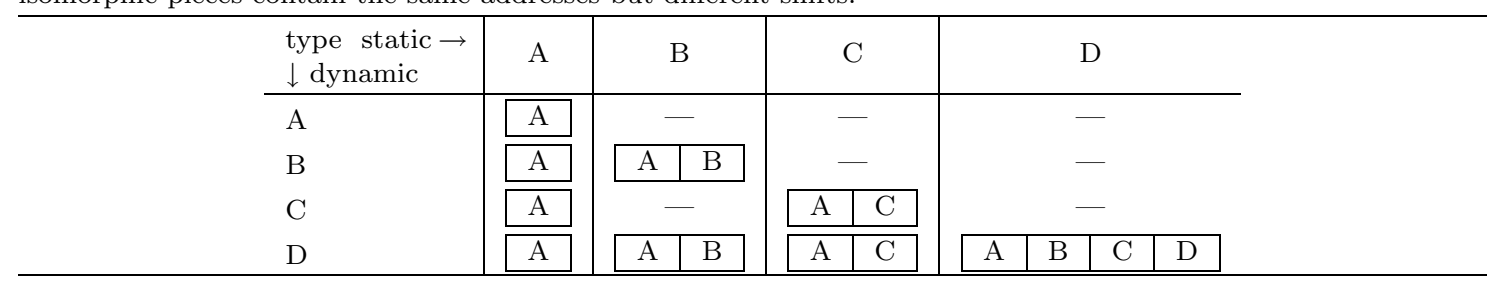

the selected method. One needs to know the position of subobject $W$, w.r.t. subobject $\tau_{d}$, noted ${ }^{6}$ $\Delta_{\tau_{s}, W}$ (Figure 3). Thus method tables have double entries for each method, an address and a shift (Figure 6 on top). On the whole, method calls are compiled by the sequence ${ }^{7}$ :

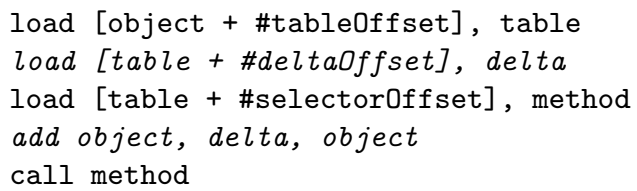

Instead of putting shifts in the tables, an alternative consists in defining a small intermediate function, called thunk ${ }^{8}$ by Ellis and Stroustrup [1990] or trampoline by Myers [1995], which shifts the receiver before calling the method:

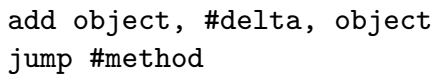

The call sequence is then the same as with SST. Thus, one saves on one access in the table for an extra direct branching. Thunks may be shared when the address and the shift are the same, and the thunk with the null shift is the method itself. The thunk could also be inlined in the method table instead of being pointed by it: one could save on $L$ cycles, but one would lose the null cost of the null shift. One must notice that the thunk may not be generated when compiling class $W$, as the shift depends on $\tau_{d}$, not only on $\tau_{s}$. Generating the thunk as a method entry point, when compiling $W$, would need an explicit upcast, i.e. a shift with a table access.

\subsection{Casting}

Subobjects make casting real: [Rossie et al. 1996] defines it as a subobject change. Notice first two basic properties of $\Delta$ :

$$
\begin{array}{lrl}
\forall \tau_{d}, T, U, V: & \tau_{d} \preceq T, U, V \Rightarrow \Delta_{T, V} & =\Delta_{T, U}+\Delta_{U, V} \\
\forall \tau_{d}, T: & \tau_{d} \preceq T \Rightarrow \Delta_{T, T} & =0
\end{array}
$$

3.2.1 Cast to dynamic type. Because of the reference variance w.r.t. static types, equality tests between two references need to equal first their types. Without loss of generality, both references must be reduced to their dynamic type: each method table must contain a shift $\Delta_{\tau_{s}, \tau_{d}}$, noted $\Delta_{\Downarrow}^{\tau_{s}}$. When the two types are in a subtyping relation, one upcast will be enough.

3.2.2 Upcast. Changing from the current subobject, with type $\tau_{s}$, to a statically known supertype $T$, needs a shift $\Delta_{\tau_{s}, T}$, which depends on $\tau_{d}$. An extra table, noted $\Delta_{\tau_{s}}^{\uparrow}$, is needed in every method table: $T$ offset in $\Delta_{\tau_{s}}^{\uparrow}$ is invariant w.r.t. dynamic type and statically known:

INVARIANT 3.2.1. Each class has an unambiguous and invariant offset in the static context of each of its subclasses.

\footnotetext{
${ }^{6}$ The notation $\Delta_{T, U}$, as all the following notations $\Delta$, implies a given dynamic type $\tau_{d} \preceq T, U$ : expliciting it would make the notation heavy.

7 Instructions added w.r.t. SST are italicized (see page 4).

8 According to Lippman [1996], thunk is Knuth spelled backwards.

RR LIRMM 2002-174
} 


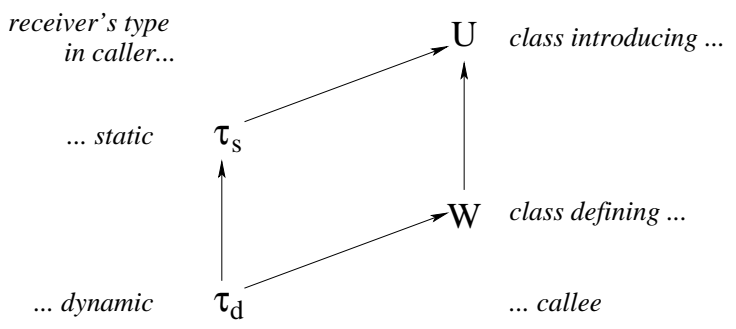

Fig. 3. Receiver's types in a method call: a shift $\Delta_{\tau_{s}, W}$ is needed

This offset is noted $i_{\tau_{s}}(T)$ and it is, unlike $\Delta \mathrm{s}$, independant of $\tau_{d}$ : then $\Delta_{\tau_{s}, T}=\Delta_{\tau_{s}}^{\uparrow}\left[i_{\tau_{s}}(T)\right]$, shortened in $\Delta_{\tau_{s}}^{\uparrow}(T)$. Instead of being a proper table, $\Delta_{\tau_{s}}^{\uparrow}$ can be inlined in method table, either by interweaving method entries and shifts, or with a bidirectional layout. Indeed, upcasts can be handled as if every target class introduces a method for upcast towards itself: but it is more efficient to put a shift instead of an address in the method table entry.

3.2.3 Accesses to attributes. The table $\Delta_{\tau_{s}}^{\uparrow}$ is also used for accessing attributes when they are introduced in a superclass of $\tau_{s}$. Let $\delta(p, U)$ be the position of an attribut $p$ w.r.t. the subobject of type $U$, and $\delta_{p}$ the offset of $p$ in the type $T_{p}\left(\tau_{s} \prec T_{p}\right)$ that introduces it (Invariant 3.1.1). Then:

$$
\delta\left(p, \tau_{s}\right)=\Delta_{\tau_{s}, T_{p}}+\delta\left(p, T_{p}\right)=\Delta_{\tau_{s}}^{\uparrow}\left(T_{p}\right)+\delta_{p}
$$

When an attribute is not introduced in the receiver's static type $\left(T_{p} \neq \tau_{s}\right)$, there is an appreciable overhead in comparison with SST:

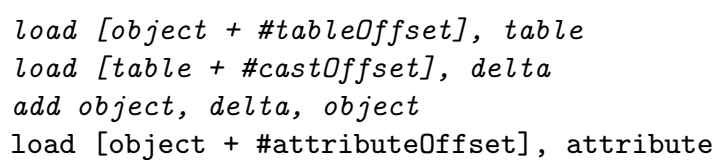

In worst case, assignment $a . x:=b . y$ needs three upcasts, on $a, b$ and between $y$ and $x$ types. Some parallelism is likely but the sequence is 5 times longer than in SST, as well in cycle number $(5 L+3$ vs. $L+1)$ as in instruction number (11 vs. 2$)$.

3.2.4 Downcast. Shifting from static type $\tau_{s}$ to a static subtype $T$ needs both a type check and the value of $\Delta_{\tau_{s}, T}$. Contrary to SST, a direct access is difficult, at least in separate compilation (see section 7.4) and some sequential search may be better. Each class $\tau_{d}$ has an association structure, e.g. a hashtable, which maps all supertypes $T$ of $\tau_{d}$ to $\Delta_{\tau_{d}, T}$ : this structure, noted $\Delta^{\Uparrow}$, can be referenced by each method table, not only by $\tau_{d}$. A downcast from $\tau_{s}$ to $T$ looks up for $T$ in the table. If $T$ is not found, a type error is signaled. Otherwise, $\Delta_{\tau_{d}, T}$ is returned and:

$$
\Delta_{\tau_{s}, T}=\Delta_{\tau_{s}, \tau_{d}}+\Delta_{\tau_{d}, T}=\Delta_{\Downarrow}^{\tau_{s}}+\Delta^{\Uparrow}(T)
$$

It must be noticed that both tables $\Delta_{\tau_{s}}^{\uparrow}$ and $\Delta^{\Uparrow}$, have the same contents, with different structures and uses: in the former, $\tau_{s}$ is statically known, whereas in the latter, $\tau_{d}$ is not (Table III). Their contents is also the same as the shifts included in the method tables or thunks. One can get advantage from static types, by associating to each subobject a new table $\Delta_{\downarrow}^{\tau_{s}}$, the restriction of $\Delta^{\Uparrow}$ to the classes between $\tau_{s}$ and $\tau_{d}: \Delta_{\downarrow}^{\tau_{s}}=\Delta \Uparrow /\left[\tau_{s}, \tau_{d}\right]+\Delta_{\Downarrow}^{\tau_{s}}$. They avoid a two step downcast:

$$
\Delta_{\tau_{s}, T}=\Delta_{\downarrow}^{\tau_{s}}(T)
$$

but the temporal advantage is small and at the cost of a memory overhead. With $\Delta^{\Uparrow}$, downcasts may be generalized to side casts (also called cross casts), where the target type $T$ is a supertype of $\tau_{d}$, but not always a subtype of $\tau_{s}$ (Figure 4 , right).

This type checking implementation is costly. Coding the specialization partial order is difficult in MI and it would not work since the problem is no more boolean: shifts are needed. A simple solution allowing a direct access is a $N \times N$ matrix, where $N$ is the class number: $\operatorname{mat}_{[}\left[i d_{T}, i d_{U}\right]$ contains $\Delta_{T, U}$ if $U<: T$, and otherwise a distinguished value meaning that there is a type error. 


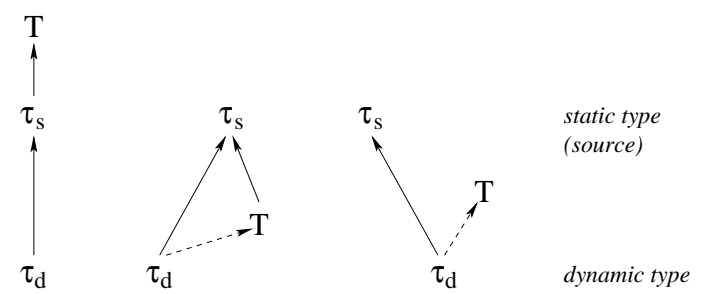

Fig. 4. Upcast (left), downcast (center) and side cast (right), to a target type $T$ : the dashed subtype relation must be checked at run-time before shifting.

Such a matrix needs $2 N^{2}$ bytes, i.e. $2 N$ bytes per class: this is a reasonable cost when there are $N=100$ classes, but it is not when $N>>1000$ (see appendix A). Class identifiers $i d_{C}$ must be computed globally for all classes $C$ : an adequat numbering allows to replace the matrix by a triangular one, i.e. by one vector per class, using vect $v_{U}\left[i d_{T}\right]$ instead of $\operatorname{mat}\left[i d_{T}, i d_{U}\right]$. This reduces the cost to an average of $N$ bytes per class, which is the worst-case cost of the coloring scheme as well in SST (see section 2.2.2) as in MI (see section 7.4) but the average cost of coloring is far lesser.

3.2.5 Monomorphic cases. When they are detected by an intra-procedural analysis, monomorphic cases simplify method calls in the same way as with SI. Casts and attribute accesses are also simplified, as all shifts are known at compile-time. Therefore, there is no MI overhead in the monomorphic cases.

\subsection{Empty subobjects optimization (ESO)}

On the basis of this uniform implementation, a simple optimization reduces the space overhead. Indeed, an exception to Invariant 3.1.3 is possible, when a subobject is empty, i.e. when the corresponding class, say $F$, introduces no attribute. It is then possible to merge the $F$ subobject with the subobject of one of its direct superclasses, say $E$. Two cases are to distinguish.

In the first case, $E$ is the only direct superclass of $F$ and $F$ introduces no method: the set of methods of $E$ and $F$ are the same. The $F$ subobject may be merged into the $E$ subobject as, without merging, the contents of both method tables, i.e. method addresses, would have been the same. Here, merging works because it is invariant w.r.t. dynamic type: $E$ and $F$ subobjects are merged in all subclasses of $F$. Multiple inheritance problems are avoided because $F$ has no more methods than $E$ : if another subclass $F^{\prime}$ of $E$, is in the same case, the subobjects $E, F$ and $F^{\prime}$ can be merged in any subclass of both $F$ and $F^{\prime}$. In some way, this merging is a property of $F$ : method tables are shared and shifts between $F$ and $E$ are avoided, as $\Delta_{F, E}=0$ and $i_{\tau_{s}}(E)=i_{\tau_{s}}(F)$, for all $\tau_{d} \preceq \tau_{s} \preceq F \prec_{\mathrm{d}} E$. The code generated for all $\tau_{s} \preceq F$ takes into account the merging of $E$ and $F$ : e.g. accessing an attribute introduced in $E$ on a receiver typed by $F$ will need no cast.

In the second case, $F$ has either more methods than $E$, or more than one direct superclassthe latter condition implies the former, as superclasses imply upcast pseudo-methods-, but the method order of $E$ is a prefix of the method order of $F$, i.e. the offsets of $E$ methods are the same in $F$. Therefore, the $E$ and $F$ subobjects may be merged in the implementation of $\tau_{d} \preceq F$, but at the only condition that $E$ is not already merged with another $F^{\prime}$, in the same $\tau_{d} \preceq F^{\prime}$. Once again, merging works in this restricted case because the prefix condition is also invariant w.r.t. dynamic type: however, $E$ and $F$ will not be merged in all subclasses of $F$. This means that merging is neither a property of $E$, nor of $F$, but only of some $\tau_{d} \preceq F$. In that case, merging allows to share method tables, not to save on shifts, neither in the code nor in upcast tables. The code generated for all $\tau_{s} \preceq F$ cannot consider that $E$ and $F$ are merged, but the data structures for some $\tau_{d} \preceq F$ may do that merging. In particular, accessing an attribute introduced in $E$, on a receiver typed by $F$, needs a cast, but the shift will be likely null.

It is always possible to construct the method order of any class, which is up to now arbitrary, as an extension of the method order of at least one of its superclasses. Therefore, almost all empty subobjects can be merged: the only exception will be when two different subobjects are merged 
Table III. Cast tables for the class $\left(\tau_{d}\right) D$ of Figure 2: $\Delta_{\Downarrow}$ is a scalar, $\Delta^{\uparrow}$ a vector, whereas $\Delta_{\downarrow}$ and $\Delta^{\Uparrow}$ are hashtables.

\begin{tabular}{l|llll}
\hline$\tau_{s}$ & $\Delta_{\Downarrow}$ & $\Delta^{\uparrow}$ & $\Delta_{\downarrow}$ & $\Delta^{\Uparrow}$ \\
\hline $\mathrm{A}$ & $\Delta_{A D}$ & {$[0]$} & {$\left[(A, 0)\left(B, \Delta_{A B}\right)\right.$} & \\
$\mathrm{B}$ & $\Delta_{B D}$ & {$\left[\Delta_{B A}, 0\right]$} & $\left.\left(C, \Delta_{A C}\right)\left(D, \Delta_{A D}\right)\right]$ & \\
$\mathrm{C}$ & $\Delta_{C D}$ & {$\left[\Delta_{C A}, 0\right]$} & {$\left[(B, 0)\left(D, \Delta_{B D}\right)\right]$} & \\
$\mathrm{D}$ & 0 & {$\left[\Delta_{D A}, \Delta_{D B}, \Delta_{D C}, 0\right]$} & {$\left[(C, 0)\left(D, \Delta_{C D}\right)\right]$} & {$[(D, 0)]$} \\
\hline
\end{tabular}

into the same subobject, in the second case of merging. In that rather exceptional case, only one merging will be possible.

There is some evidence that empty subobjects are quite common and that this simple optimization is essential (see appendix A). An allusion in [Lippman 1996 $]^{9}$ suggests that it is used in actual compilers but it has not been widely noticed, as most of benchmarks in the literature do not include any information on attribute number [Sweeney and Burke 1998; Gil and Sweeney 1999; Eckel and Gil 2000] and some compilers don't know it, G++ for instance.

\subsection{Evaluation}

Multiple inheritance overhead is rather large.

Dynamic memory. In each object, the overhead is equal to the number of indirect superclasses of the object class, which introduce at least one attribute.

Static memory. The number of method tables is no more linear in the class number $N$, but worstcase quadratic whereas total size is no more quadratic, but cubic: $\Sigma_{C}\left(\Sigma_{C} \preceq D M_{D}\right)$. However, the formula must be corrected to take into account the empty subobjects optimization: $\Sigma_{D}$ is restricted for those $D$ which are not merged with some $E$, with $C \preceq D \prec_{\mathrm{d}} E$. Precise statistics confirm both the overhead of this implementation and the benefits of empty subobjects. In a context of mainly "non virtual" inheritance, [Driesen 1999; Driesen and Hölzle 1995] report a ratio larger than 3 on the table sizes, w.r.t. the size of tables in $\operatorname{SST}\left(\Sigma_{C} M_{C}\right)$. Statistics in appendix show that the ratio may exceed 6 with standard implementation, but that it is reduced to 4 with ESO. When taking into account the shifts, in the tables or in the thunks, the ratio climbs again to 6 . Thunks seem roughly equivalent to putting shifts in method tables: there are less thunks than tables entries, they need two words instead of one and the code sequence is two instructions shorter. Statistics show that they are more costly for large hierarchies (see appendix A).

Time efficiency. A shift is needed each time an assignment or a parameter passing is not with constant static types. It imposes an extra access to method table. The real impact on method calls is more questionable: shifting could be done within processor's latencies or in parallel. Experiments by [Driesen 1999] seem to give a small advantage to thunks. But this conclusion is based on benchmarks mainly constituted of classic $\mathrm{C}++$ programs making an heavy usage of "non virtual" inheritance: in this particular case, shifts are mostly null and the thunk is the method itself. So, although it does not allow to conclude about plain MI, it seems that shifts have a sensible overhead. This is not surprising since, even if the cycle numbers are almost equal, this is at the price of some parallelism, and it is likely that shifts take the place of some other code sequence. Furthermore, constant-time downcast needs very large tables. The main drawback of this implementation is that the overhead is the same when one does not use MI: separate compilation is unable to foresee that a given class will be always specialized in SI.

\section{ALTERNATIVES FOR MULTIPLE INHERITANCE}

The complexity of the previous implementation explains part of the reluctance to multiple inheritance as well as some unsound $\mathrm{C}++$ features [Cargill 1991; Waldo 1991; Sakkinen 1992]. Various

\footnotetext{
9 My recommendation is not to declare nonstatic members within a virtual base class. Doing that goes a long way in taming the complexity (p. 139). In general, the most efficient use of a virtual base class is that of abstract virtual base class with no associated data members (p. 101).
} 


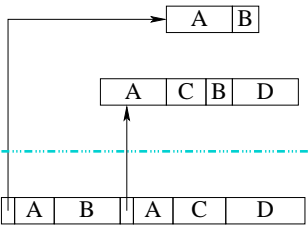

(a)

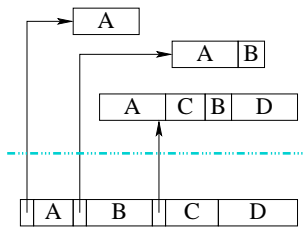

(b)

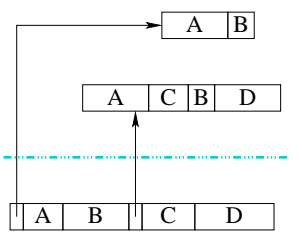

(c)

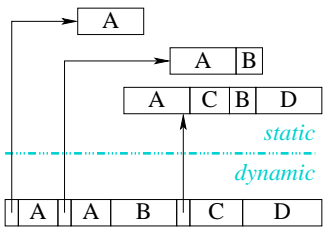

(d)

Fig. 5. Non virtual inheritance: pure NVI and repeated inheritance (a), mixed NVI when $D \prec_{\mathrm{nv}} B, C \prec_{\mathrm{v}} A$ (b), in ideal (c) and actual (d) implementations when $B \prec_{\mathrm{nv}} A$ and $C \prec_{\mathrm{v}} A$.

alternatives have been searched for: the most radical, proposed by the SMALL EIFFEL compiler that we will examine later (see section 7.3.6), avoids method tables at the price of a global compilation [Zendra et al. 1997]. In this section, we examine some small variations around the standard implementation. They consist either in searching a MI implementation without overhead when used only in SI (section 4.1), or various compromises between time and space efficiencies (sections 4.2 and 4.3). Eventually, attribute implementation can be reduced to methods (see section 4.4).

\section{$4.1 \mathrm{C}++$ non virtual inheritance (NVI)}

As previously noticed, the main drawback of standard MI implementation is that its overhead does not depend on an effective use of MI. C++ "non virtual" inheritance is an answer to this problem. It is expressed by not annotating superclasses with the keyword virtual. The implementation is more efficient but it is sound only for arborescent inheritance (AI), i.e. when the superclasses of any class form a tree.

4.1.1 Pure NVI. NVI is better described by its implementation than by its semantics [Ellis and Stroustrup 1990; Lippman 1996]. A class without superclass is implemented as in SST. When a class has one or more direct superclasses, the instance layout consists of the concatenation of the instance layouts of all direct superclasses. The attributes introduced by the class are added at the end and the method table of the class extends the method table of one of its direct superclasses: the choice of this direct superclass is a matter of optimization and it defines a new relation $\prec_{\mathrm{e}}$, a subset of $\prec_{\mathrm{d}}$. Thus, the main difference is that there is no proper subobject for the class itself and all $\Delta_{T, U}$ are invariant w.r.t. $\tau_{d}$. Therefore, upcasts and attribute accesses are truly static in the sense that they do not need table accesses. However, the code generated for method calls is the same as with standard MI implementation. The shifts are always needed but they are more often null: thunks make most of them disappear in practice. Moreover, thunk elimination is possible: the overriding method expects a value of self typed by the class introducing the method and two static shifts are made in the caller and in the callee [Lippman 1996, p. 138]. Alternatively, general support for multiple entry points avoids explicit thunks: an entry point is generated for each $V$ such that $W \preceq V \preceq U$. This is now possible because the shift is independant from $\tau_{d}$.

The layout is a direct generalization of SST and, in case of SI, it is exactly the same as with SST, with only one subobject and there is no shift at all with thunks. In the general case of arborescent inheritance, the number of subobjects or method tables is equal to the number of superclasses which are roots, i.e. without superclass.

4.1.2 Arborescent vs. repeated inheritance. The flaw of non virtual inheritance occurs when inheritance is no more arborescent, i.e. when the inheritance graph contains undirected cycles, as in Figure 2. It consists in what we call repeated inheritance, in the sense that some subobject is repeated in the object layout: in Figure 5-a, this is the case for $A$ which is present in both subobjects of $B$ and $C$. In case of repeated inheritance, the table number of NVI implementation becomes exponential in the number of superclasses, as it is the number of paths from the considered class to its root superclasses.

4.1.3 Mixing "virtual" and non virtual inheritance. This is the usual programming style in $\mathrm{C}++$. Inheritance edges, i.e. $\prec_{\mathrm{d}}$, are partitioned in two sets, $\prec_{\mathrm{v}}$ and $\prec_{\text {nv }}$, according to whether the keyword virtual is used, or not. The layout of a class is made of two parts. The non virtual RR LIRMM 2002-174 
part is computed as for pure NVI, but the concatenation on direct superclasses is restricted to the $\prec_{\text {nv }}$ relationship: thus $\prec_{\mathrm{e}}$ is a subset of $\prec_{\mathrm{nv}}$. The virtual part is made of the non virtual parts of all superclasses, either direct or indirect, which are not reachable through $\prec_{\text {nv }}$ paths. Therefore, the object layout consists of subobjects groups: each group is made of subobjects in a fixed relative position, whereas groups have arbitrary relative positions. Of course, all partitions of $\prec_{\mathrm{d}}$ are not sound. $\prec_{\text {nv }}$ must imply arborescent inheritance in order to avoid repeated inheritance. Furthermore, with repeated inheritance, mixing may be ambiguous, e.g. when a class $C$ is repeated in some direct superclass, and virtual in some other. However, the implementation described here (Fig. 5-c) is ideal as it differs from actual C++ specification by examples [Koenig 1998, p. 165]: although virtuality is an annotation of inheritance edges, the $\mathrm{C}++$ object model makes it an annotation of classes. Therefore, a superclass inherited twice, through virtual and non virtual edges, is repeated (Fig. 5-d) and both links must be virtual in order to avoid repeated inheritance (b).

4.1.4 Casting. In case of arborescent inheritance and pure NVI, the relative positions of two subobjects whose types are related by subtyping don't depend on the dynamic type. Thus upcast is unambiguous and, when it is needed, the shift is static, without accessing the table. Downcast can also be made in a very close way to the SST coloring scheme, as $\Delta_{\tau_{s}, T}$ is independant of $\tau_{d}$. One needs only that each subobject implements the coloring scheme reduced to the types corresponding to the subobject. Only side casts need the MI technique.

On the other hand, repeated inheritance makes casting ambiguous. For upcasts, the ambiguity is on the target and intermediate upcasts may be needed to remove it. As for downcasts, the ambiguity is on the source and checks must be repeated as many times as the source is. Side and dynamic casts are not always possible.

Mixing virtual and non virtual inheritance makes the problem even more complicated. [Ellis and Stroustrup 1990, section 10.6c] forbids some cases. However, according to the specifications of dynamic_cast [Koenig 1998], the effective implementation seems to use both $\Delta_{\downarrow}^{\tau_{s}}$ (in case of repeated inheritance) and $\Delta^{\Uparrow}$ (for sidecast). If the target is unambiguous in one of the two tables, the cast succeeds: no static prohibition is necessary.

4.1.5 Evaluation. Non virtual inheritance has the great advantage of presenting an overhead only when one uses MI (as long as thunks are used). But repeated inheritance is a major semantic drawback. A sound semantics is possible by mixing virtual and non virtual inheritance, but it is a matter of either hand made optimization, or global analysis, as separate compilation cannot predict that $B$ and $C$ will not have a common subclass and which one must use the virtual keyword. Only a subsequent diagnosis, when defining $D$, is possible, or an automatic devirtualization analysis on the whole program (see section 7.3.2). From a programmer point of view, mixing virtual and non virtual inheritance may be quite complicated and it even worsens with inheritance protections. It could be possible to restrict the use of NVI to AI: defining $D$ would be forbidden. But it would be a limitation to reusability. Moreover, when the class hierarchy has a root, Object, the class of all objects, AI reduces to SI.

Besides its semantic flaw, pure NVI is not less costly than standard MI implementation, in all generality: NVI complexity is indeed exponential in the worst-case (see appendix A). Furthermore, empty subobjects optimization applies only to classes without non virtual direct superclasses.

4.1.6 Criticizing $\mathrm{C}++$. NVI comes from Simula [Krogdahl 1985]. Multiple inheritance in $\mathrm{C}++$ has been commented and criticized [Cargill 1991; Waldo 1991; Sakkinen 1992], often for opposite reasons. [Cargill 1991] criticizes "virtual" inheritance and it is significant that Waldo's answer [1991] is based on an example which needs rather MST than plain MI (see also note 9, page 11). The main criticism may be that understanding the language goes through understanding the implementation, e.g. by reading [Lippman 1996]. NVI gave rise to some attempts to base its semantics on models of subobjects [Rossie et al. 1996; Snyder 1991]. 

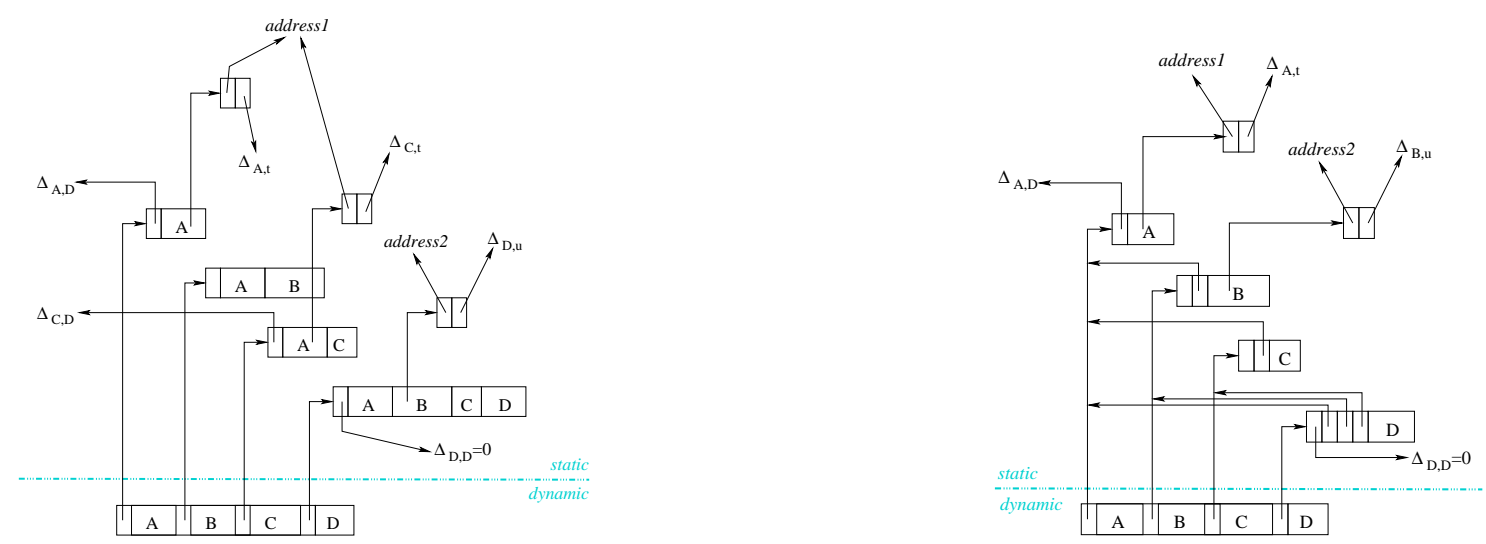

Fig. 6. Standard and compact method tables

\subsection{Compact method tables}

Another drawback of standard MI implementation is its static space overhead: table size is cubic instead of quadratic in SST. Two obstacles forbid to share the tables of different static types for the same dynamic type: shifts and variant method offsets. The first point can be reduced by a two step shifting, as for attributes (5) and downcast (6). The second point is solved by applying to methods Invariant 3.1.1, which rules attributes. Therefore, the method table associated to a static type contains only the methods introduced by the type, not inherited methods. A method call must begin with an upcast towards the type which introduces the method, as with attributes (5), at least when $T_{p} \neq \tau_{s}$. The cost of this technique is exactly the sum of the costs of upcasts and method calls: thus, the call sequence has 8 instructions and $4 L+B+2$ cycles. Instead of getting the second table from the cast object, one can use pointers between the different method tables (Figure 6, right). This allows some parallelism and reduces the cost to $3 L+B+1$ cycles:

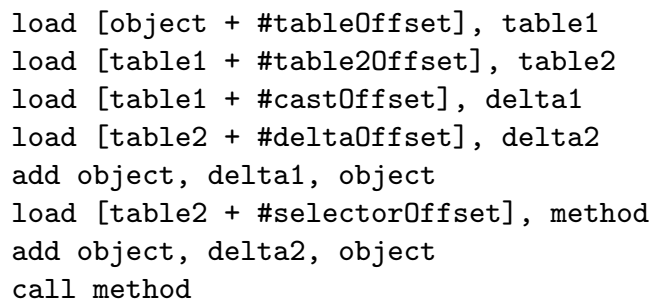

In that case, the two step shift may indifferently go through either $\tau_{d}(6)$ or $T_{p}(5)$. A minor overhead is that upcast structures are implemented twice, as shifts between subobjects and as pointers to method tables.

Evaluation. This technique reduces static space overhead to the only cost of doubling the upcast tables size, together with 3 extra instructions for each method call such that $\tau_{s} \neq T_{p}$. The objective of this compact implementation is to reduce table size, i.e. static memory, but it may be hindered by this sensible augmentation of the code. Only the first case of empty subobject optimization works: merging is possible when both the subobject and its method table are empty. Moreover, time efficiency is reduced: $L$ extra cycles underestimates the effective extra overhead. Anyway, it was worth-while to convince oneself that standard implementation offers, in some way, the best compromise between time and static memory. Various other compact schemes exist, but no one is better than this simple one.

\subsection{Less indirections with VBPTRs}

Contrarily to previous variants which try to reduce the static memory cost, some optimizations aim to reduce time overhead, at the price of dynamic memory. The best way to do it is to move the upcast tables $\Delta_{\tau_{s}}^{\uparrow}$ from the method tables to the objects themselves. In a second step, shifts RR LIRMM 2002-174 
common to all instances may be replaced by pointers to subobjects, proper to each instance. In the $\mathrm{C}++$ jargon [Ellis and Stroustrup 1990], they are called virtual base pointers (VBPTR) and, according to [Sweeney and Burke 1998], this technique is used in several C++ compiler. As a matter of fact, upcasts are very frequent, as well for accessing attributes as for polymorphic assignments. They are viewed by standard implementation as methods, which impose a method table access (see section 3.2.2, page 8). VBPTRs amount to view upcasts as attributes, but they are implemented in each subobject.

load [object + \#castOffset], object

load [object + \#attributeOffset], attribute

A similar optimization will consist in replacing $\Delta_{\Downarrow}$ by a pointer to $\tau_{d}$ subobject in the object layout [Sweeney and Burke 1998].

Evaluation in standard implementation. As method tables, $\Delta_{\tau_{s}}^{\uparrow}$ tables are, in worst case, quadratic in number and cubic in size. Therefore, with standard MI implementation, in each instance, the number of VBPTRs is quadratic in the number of superclasses, and the worst case occurs with SI: a $n$ class chain induces $n(n-1) / 2$ VBPTRs. In constrast, assuming a uniform distribution of attributes introduced in each class, the number of attributes may be considered as linear in the number of superclasses: thus, VBPTRs may easily occupy more space than attributes (see appendix A). Furthermore, VBPTRs are compatible only with the first case of empty subobjects merging: indeed, in the second case, the $F$ subobject would not be empty since it must contain a VBPTR to the $E$ subobject (see section 3.3 ). As for the pointer to $\tau_{d}$, the overhead is smaller, as it is equal to subobject number, but the gain is also smaller as shifts to the dynamic type are less frequent. On the whole, VBPTRs large dynamic overhead is not counterbalanced by a small temporal gain.

A by-product of VBPTRs is the ability to implement instance classification, a monotonous special case of instance migration (e.g. change-class function in CLOS) when the target class is a subclass of the source class. Indeed, with VBPTRs, the object layout needs not to be connected: specializing an object's class amounts to add subobjects and change pointers to method tables.

Evaluation in NVI and optimizations. When mixing virtual and non virtual inheritance, the overhead is far smaller: this explains why actual compilers use VBPTRs. The reason of this smaller overhead is twofold. Firstly, with NVI, a class can reuse the VBPTRs of its direct non virtual superclasses, which is not the case with $\Delta_{\tau_{s}}^{\uparrow}$ tables since they are relative to $\tau_{s}$. Secondly, one may distinguish two kinds of VBPTRs: e-VBPTRs are essential because they reference superclasses reachable only through $\prec_{\mathrm{v}}$, whereas i-VBPTRs are inessential and can be replaced by a static shift w.r.t. an e-VBPTR. Implementing only e-VBPTRs adds one extra shift to upcast, but no overhead for attribute access as the shift may be added to the offset at compile-time. Furthermore, pointers to $\tau_{d}$ are required only in root (w.r.t. $\prec_{\text {nv }}$ ) subobjects. As against this, instance classification is no more possible as subobjects may have fixed relative positions.

Sweeney and Burke [1998] propose a general framework for analyzing the space overhead of various object layout, according to the repartition of data between class tables and object layout. [Gil and Sweeney 1999; Eckel and Gil 2000] present some statistics on the VBPTR cost in some large benchmarks and they propose optimizations aiming to reduce VBPTR overhead. Some of them are global and compare badly with techniques used in global compilation, whether coloring (see section 7.4) or SMALL Eiffel (see section 7.3.6), which do not need shifts. Other optimizations are usable in separate compilation: they use bidirectional tables (see section 5.1). On the whole, with an optimal balance of virtual and non virtual inheritance, the dynamic overhead of VBPTRs remains important, but it is smaller than the number of subobjects (see appendix A).

\subsection{Attribute accessors}

Some languages behave towards attributes and towards methods in a very close way. In CLOS [Steele 1990], attributes are accessed through special read and write methods (generic functions in the CLOs jargon) called accessors. In SATHER [Szypersky et al. 1994], an attribute contributes to the class type by its accessors signatures. In EIfFEL [Meyer 1992; 1997], a method without 

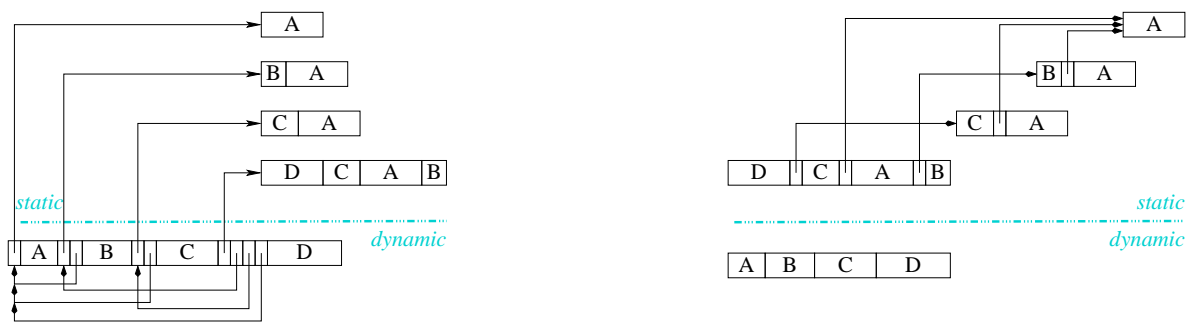

Fig. 7. Implementation variants with VBPTRs (left) and inline cache of method tables (right).

argument may be overridden as an attribute. Obviously, attribute implementation can be encapsulated in accessor methods: when an attribute position change in a subclass, it needs only to override its accessors. Thus, attribute offsets don't matter.

Two variants must be considered: either accessors are actually defined as methods or they are simulated, in which case method table contains attribute offsets instead of accessor addresses. Simulating accessors avoids a true method call: the access cost is the same as with standard implementation, in the general case $\left(T_{p} \neq \tau_{s}\right.$, page 9$)$. In a second step, attribute offsets can be factorized if attributes are grouped by subobjects, with Invariant 3.1.1. Method tables contain the position of subobjects w.r.t. the object address. The access code is now exactly the same as in standard implementation, when $\tau_{s} \neq T_{p}$.

In some way, simulating accessors is only a catch since it brings back to a subobject implementation. Moreover, shifts are needed in every case, even when $\tau_{s}=T_{p}$. In order to save shifts when accessing self, Myers [1995] proposes a double compilation of each class: in the first one, self attributes are compiled with shifts, whereas in the second one, they are compiled without shifts, assuming that the subobject position will be preserved in all subclasses (e.g. if the class is only specialized with SI). The appropriate version is chosen at link-time.

However, true accessors may be needed for some features as redefining a method without parameter as an attribute (EIFFEL) or implementing the full specifications of the Clos keyword :allocation (see section 6.3).

Nevertheless, accessors are a solution for attributes only when the question of methods has been solved. If the object layout may be constituted of a table where attribute offsets don't matter, a direct access to methods or attribute offsets is needed. Thus this is not an alternative in itself but we will see different ways to incorporate accessor simulation in a general framework, either preserving object reference variance (see section 5.1.3) or recovering SST invariance, with a global computation (see section 7.4) or a flow of method tables (see section 5.2.2). Attribute accessors can be used with any method implementation.

\subsection{Inline cache for method tables}

A radical alternative to standard implementation consists in moving the links between object layout and methods tables from data structures to program data flow. Any object value is then twofold: the object itself and its method table. One can think of it as if the compiler did replace any variable by two variables, one for the object, the other for the method table. Of course only polymorphic variables are concerned: monomorphic variables, typed by a primitive type, don't need tables. The object layout consists only of the fields for the attributes, almost doubled because of the tables but without any pointer to method tables or VBPTRs. Therefore, attributes should be accessed by simulating accessors. A secondary consequence is almost doubling method parameters and returned values.

As for method tables, they have the same structure as in standard implementation, with VBPTRs in the method tables of the same $\tau_{d}$ which are all linked with each other. Invariant 2.1.1 is respected for the object part of the value, whereas Invariant 3.1.3 rules the method table part. All the pointer adjustments needed by standard implementation, either for casting or for the receiver, are done on the method table in such a way that the method table part of a value is always the table corresponding to its static type. 
In this approach, any variable $\mathrm{x}$ is twofold: $\mathrm{x}$ itself which references an object and the method table tablex. In the assignment $\mathrm{x}:=\mathrm{y}$, one gets the appropriate table from tabley:

load [tabley + \#deltaOffset], tablex

move $y, x$

Method call to $\mathrm{x}$ follows:

load [tablex + \#selector0ffset], method

call method

Evaluation. Maintaining a systematic flow of method tables in parallel of the data flow seems at first glance a time efficient solution, at the only cost of almost doubling memory space, as well dynamic as static, in the code or in the data structures. However, the actual overhead could be less than this pessimistic view. Many data are monomorphic and this technique would have no influence on them. Dynamic memory overhead will be partially counterbalanced by the gain of all pointers, either to method tables or VBPTRs. As a matter of fact, the proposed implementation is as time efficient as standard implementation with VBPTRs, whereas VBPTRs and pointers to method tables are often more than doubling dynamic memory (see appendix A).

Increasing the code size with extra parameters is counterbalanced by the fact that there is no more need to access the object to get its method table when needed: moreover, an optimized compiler will remove all accesses to tables which are not used. This is also a good technique for implementing universal types, i.e. common superclasses of primitive types and standard classes, which avoids using wrappers as in JAVA. However, the main drawback will be collections, i.e. arrays, since polymorphic arrays will double. As a matter of fact, this technique does not seem to have been envisaged.

\subsection{Comparisons}

The main drawback of standard MI implementation is that its overhead does not depend on the fact that one uses MI, or not. NVI is a way to avoid this drawback, at the detriment of semantics. A general solution could be that languages allow to express the fact that a class should not be specialized with MI or, more generally, with non-arborescent inheritance: it would be at the detriment of reusability.

Accessor simulation coupled with Myers' double compilation is a better alternative since there is no detriment at all, but the benefits are only when accessing self attributes: method calls are not concerned, but thunks would cancel the overhead in case of SI. It is then a good solution when attributes are mostly encapsulated, à la SMALLTALK. Other alternatives seem hopeless: compact method tables add a time overhead and may be unable to effectively gain on static memory, whereas VBPTRs can be envisaged only in a context of massively NVI. However, as VBPTRs are commonly used in actual $\mathrm{C}++$ compilers (Microsoft and SUN compilers, but not GNU), caching method tables could be considered as a valuable alternative.

\section{SINGLE INHERITANCE AND MULTIPLE SUBTYPING (MST)}

Between the two extreme cases, is the middle case where classes are in SI but types are in MST, whilst class specialization remains a special case of subtyping. This is typically the case of JAVA and some other languages have a very close type policy: THETA [Myers 1995; Day et al. 1995], as well as all languages designed for the Microsoft platform DotNET, C\# [Microsoft 2001] or EIFFEL\# [Simon et al. 2000]. The specification is the one of JAVA [Gosling et al. 1996; Grand 1997]: the extents relation between classes is single, and the implements relation between classes and interfaces and the extents relation between interfaces are multiple. Only classes may define attributes together with method bodies and may have proper instances: interfaces are abstract classes and define only method signatures.

In such a framework, the SST invariants 2.1.1 and 2.1.2 cannot be verified by interfaces: more precisely, a method introduced by some interface will be implemented at different offsets in the different classes which implement the interface. However standard MI would be too complicated, as both invariants could be verified by classes. Hence, two kinds of solution can be designed, according to whether one simplifies MI implementation or one complicates SST one. 


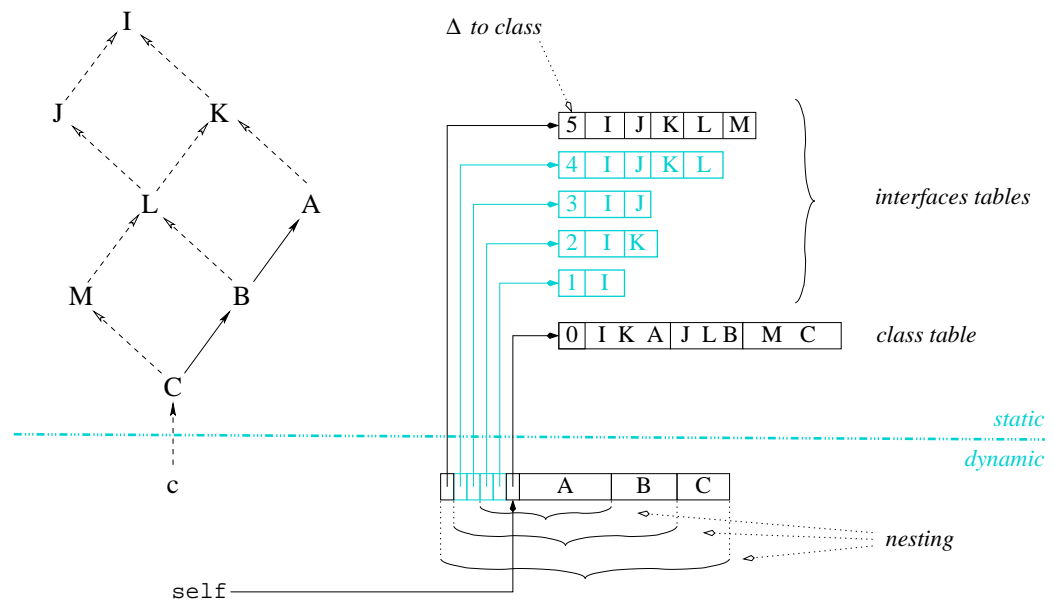

Fig. 8. Single inheritance and multiple subtyping: multiple inheritance variant, with 3 classes $A, B, C$ and 5 interfaces, $I, J, K, L, M$. In grey, interface tables saved by sharing.

\subsection{Multiple inheritance variant}

Standard MI implementation can be notably simplified in the present case. Of course, all interface subobjects are empty but as all interfaces are likely to introduce some method, only the second case of subobject merging can work (see section 3.3). A more specific presentation is better.

5.1.1 Principle. Starting from standard implementation, the first step consists in conciliating different method tables with attribute invariance. Due to attribute invariance, only one method table is needed for the classes. Due to interface specification, interface subobjects are empty. Therefore all pointers to method tables can be grouped in a header and object layout is bidirectional: positive offsets are for the attributes, negative offsets are for interface tables, and offset 0 points to the class table. Each interface table begins with the offset of the pointer to this table, which stands for $\Delta_{\Downarrow}^{\tau_{s}}$ : this value will serve to shift the receiver in a method call, when it is typed by an interface. When the receiver is typed by a class, no shift is needed as any entity typed by a class points to offset 0 , whereas entity typed by an interface points to the offset corresponding to the interface.

In a second step, one can order interface tables, in the header, in such a way that the superclass implementation is nested inside the subclass one: the subclass add new interfaces at negative offsets and new attributes at positive offsets (Figure 8).

INVARIANT 5.1.1. Superclass implementation is nested inside subclass implementation: interface shifts $\left(\Delta_{\Downarrow}^{\tau_{s}}\right)$ are invariant by inheritance, i.e. w.r.t. $\tau_{d}$.

The third step consists in factorizing interface tables. Sharing tables needs to take a specific convention on the way method tables are built (see section 3.3): each interface and class orders its super-interfaces in some arbitrary order, e.g. depth-first one, in such a way that, in case of SST, the superclass order is a prefix of the subclass order. Methods are also grouped in tables according to this order, and method offsets are invariant in each group, as with Invariant 3.1.1. Two interfaces may share the same table when the superinterface order is a prefix of the subinterface order. In Figure 8, class table is shared by $K$ and interface table of $M$ is shared by all other interfaces.

Code for accessing attributes is the same as in SST, as well as method calls when the receiver is typed by a class. When the receiver is typed by an interface, the code is the same as in MI, with the difference that deltaOffset does not depend on the method.

5.1.2 Casting. Several cases must be considered:

(1) from class to class, it is done exactly as with SST, and from interface to interface, as with MI;

(2) from class to interface, for an upcast, shift is static (constant and invariant w.r.t. dynamic type, thus without table access) thanks to nesting; a sidecast will need the table $\Delta^{\Uparrow ;}$ 

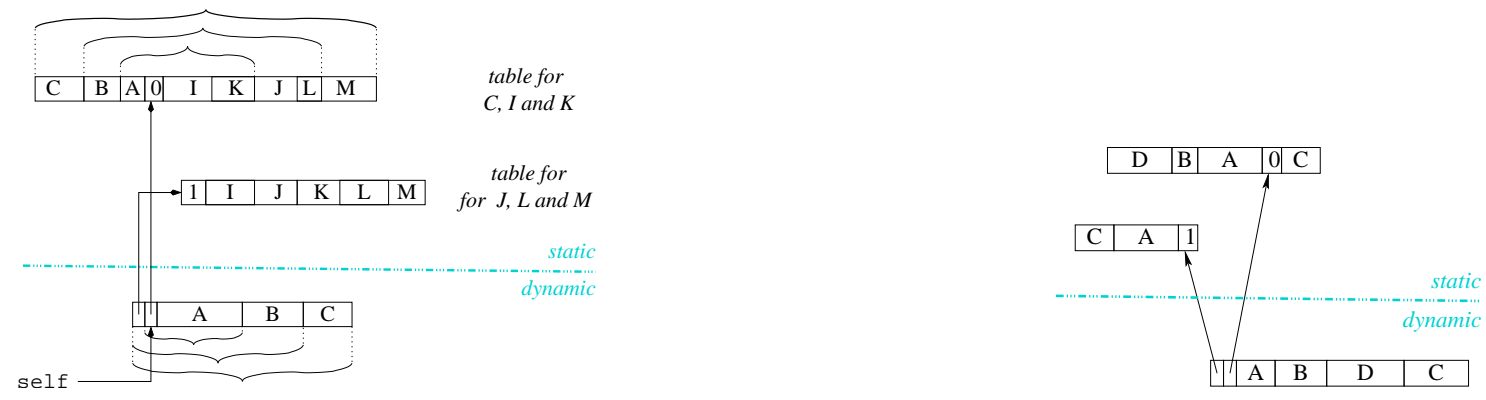

Fig. 9. Theta techniques, for examples of Figure 8 (left) and Figure 2 (right), where $B$ is considered as primary.

(3) from interface to class, the shift is in the table header, but there are two cases: a method call to a receiver typed by an interface does not need any type checking, but downcast needs type check, which is done as in SST.

When a case reduces to MI, $\Delta^{\Uparrow}$, and possibly $\Delta^{\uparrow}$, tables are needed, respectively in the class and in the interface tables.

5.1.3 Case of Theta. The language Theta uses a very close technique with some optimizations [Myers 1995]. The basic idea is to extend object layout bidirectionality to method tables ${ }^{10}$. The positive part contains methods declared in interfaces, when negative part contains methods introduced in a class (Figure 9). On the example, the sharing is not better than with the first variant (Figure 8), but it is intuitive that bidirectionality might improve sharing: without bidirectionality, methods introduced by a class forbid any sharing for the subclass interfaces. Myers [1995] proposes also an optimizing algorithm for computing tables.

5.1.4 Application to multiple inheritance. Myers [1995] proposes two extensions of his technique to MI. Both are based on the principle that, when a class has more than one direct superclasses, one of them is considered as primary, and the other are secondary. Implementation will respect the nesting principle for the primary superclass only. Obviously, the best choice of the primary superclasses is a matter of global optimization (see section 7 ).

Variant with method copy. The first variant is based on the code copy of all methods and attributes defined in secondary superclasses: code sharing is done only with direct and indirect primary superclasses (see section 7.3.1). The technique is sound only for language with a strict attribute encapsulation, à la SMALLTALK: if this is not the case, accessors are needed (see section 4.4). Once attributes and methods have been copied, secondary superclasses can be treated as if they were interfaces (Figure 9).

The main advantage is that MI costs only when it is used and it is always as efficient as standard inheritance, at least from a dynamic point of view: the static overhead of copying needs some assessment. But copying method code is not compatible with separate compilation. According to the author, experiments show a sensible improvement w.r.t. $\mathrm{C}++$ : on the example, the result is as good as with NVI (Figure 5).

Variant with accessor simulation. Instead of copying attributes, they are accessed by simulating accessors, and shifts to subobjects are added in the method tables (see section 4.4). The double compilation saves many shifts but thunks are now needed since methods may be defined in secondary superclasses. As a whole, this is certainly a good alternative to standard implementation, as efficient as NVI but without its flaws.

\subsection{Single subtyping variant}

The other approach consists in starting from SST implementation and extending it to interfaces.

\footnotetext{
$\overline{10}$ Bidirectionality is also found in [Eckel and Gil 2000], where a positive or negative direction is arbitrarily assigned to classes without superclasses: specializing two classes with opposite direction saves on VBPTRs (see section 4.3). Bidirectionality seems to originate in [Pugh and Weddel 1990] (see section 7.4).
} 

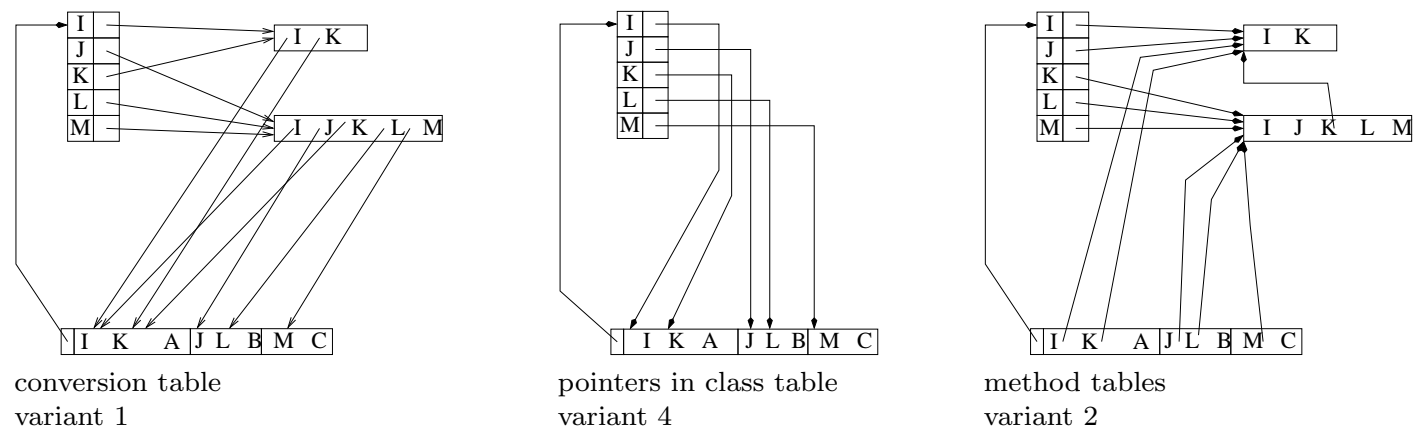

Fig. 10. Multiple subtyping and single inheritance: single subtyping variants.

5.2.1 Principle. Reference Invariant 2.1.1 is then conserved in any case, whereas Invariant 2.1.2 is always restricted to class-typed entities. Object layout and class method tables are the same as with SST, but some data must be added in method tables to deal with the case of interface-typed entities. For each interface implemented by a class, a data structure is needed to find methods and many techniques can be envisaged (Figure 10):

(1) offset conversion tables between interface and class,

(2) first class interface method tables,

(3) if methods are grouped by class and interface, with an invariant order in each as with Invariant 3.1.1, shifts to each group are sufficient,

(4) shifts of (3) can be replaced by pointers to method groups.

But it does not seem possible to have a constant-time direct access to those data structures for a given interface. Thus an association structure such as a hashtable, like $\Delta^{\Uparrow}$, is needed in the class method table.

Starting for this general framework, some optimizations are possible. Time optimizations will consist in caching interface tables as long as the reference to the object does not change: so one access to the hashtable could serve for several method calls (see section 5.2.2). Space optimizations are based on sharing data, between interfaces and even between classes (inheritance), for variants 1 and 3. Variant 2 allows sharing only for the same class. Variants 1 and 2 have the advantage that they deal with all the methods of the interface as a whole, whereas variants 3 and 4 consider only the methods introduced by the interface. Caching will be more efficient with the former.

As for casting, it is realized mostly as in SST. Due to reference invariance, only downcasts and dynamic type check must be considered. When the target type is a class, any SST technique applies. When the target is an interface, one will use the interface association structure in a boolean way, or any other MI boolean technique.

5.2.2 Constant-time variant, with inline table cache. Implementation of interface-typed entities may be a good opportunity for inline cache of method tables (see section 4.5). Indeed, the space overhead of this technique would concern only interfaces, which are presumed to be less used than classes for typing program entities.

\subsection{Application to JAVA.}

According to its specifications [Gosling et al. 1996; Grand 1997], JAVA could adopt either MI variant or SST variant. However, the language implementation seems to be constrained by the specifications of its run-time environment, the Java Virtual Machine (JvM) [Meyer and Downing 1997].

5.3.1 Interface typed receiver. In the JvM, a specific operation, invokeinterface, addresses the case of method call to an interface typed receiver. [Alpern et al. 2001] present a state of the art of existing implementations, which mostly use dynamic typing techniques (see section 7.2). As the problem of searching a method on an interface-typed receiver can be reduced to the problem of searching an interface table, various techniques come back to the approach of a large class-method 
table, possibly transformed in a smaller class-interface table. This is the case of virtual machines Cacao [Krall and Grafl 1997] and Sable [Gagnon and Hendren 2001]. Other techniques as inline cache, possibly polymorphic [Hölzle et al. 1991], are quoted. In Jalapeño, [Alpern et al. 2001] associates to each class a fixed-sized hashtable containing either a method address, or a decision tree indexed on interface identifiers.

5.3.2 Subtyping checks. Dynamic class loading forbids the simple approach of Schubert's numbering. However, the [Cohen 1991] coloring variant is incremental and it is used in Jalapeño [Alpern et al. 2001]. When the target type is an interface, this technique doesn't work and a boolean class-interface matrix associates to each class the interfaces implemented by the class. Some virtual machine implementations seem to have adopted a hashtable technique as well for subtyping test as for invokeinterface [Meyer and Downing 1997, chapitre 9].

5.3.3 Other JAVA features. A problem of SI is the implementation of general system-defined functionalities which require some implementation in the object layout. A typical example is synchronization, which needs locks in the objects. As the instances of any class may be synchronized, a class Synchronized-Object would have been incompatible with SI, and an interface would fail to add some implementation in the objects: so the functionality is introduced in Object. Implementing locks with attributes would be a simple solution, but this attribute should be defined in Object: the overhead would be for all objects, even when they don't use synchronization ${ }^{11}$. Implementing them in object header would be the same as an attribute defined in Object. [Bacon et al. 2002] proposes an approach based on statically distinguishing classes which need synchronization from classes which need not: locks are then defined as an extra attribute in the former only. In order to maintain the offset invariance required by the synchronization code, this attribute is implemented at a negative offset, unlike other attributes. However, as instances of non-synchronized classes may be synchronized as well, a global data structure, called a lock nursery, is dedicated for it. Statistics show that most synchronized objects are instance of synchronized classes, so the gain of locks for non-synchronized classes more than counterbalances the overhead of the lock nursery.

\subsection{Application to multiple inheritance: mixins}

Literature on mixins is rather large [Stefik and Bobrow 1986; Bracha and Cook 1990; Ancona et al. 2000; Ernst 2002], but they are still missing a precise definition. They consist in a variation on the notion of abstract classes, in a less abstract way than the interface notion of JAVA. They are often presented as a way, either to avoid MI, or to discipline its usage. Our definition is the following. A mixin is an abstract class, in the SMALLTALK sense, i.e. a class without proper instances, but, contrarily to JAVA's interfaces, a mixin may have attributes and method bodies. Mixins are aimed at defining functionalities which are presumed orthogonal to classes and which can be mixed to them without conflict risk. We will not discuss here this hopeless objective but only propose a specification of specialization in a world where classes and mixins can be mixed. (1) classes are in SI: a class cannot specialize more than one class. (2) a mixin can specialize at most one class, but no mixins: mixins must be thought of as unrelated functionalities, but some of them may apply only to instances of some precise class. (3) a class may specialize as many mixins as one wants. (4) when a class specializes a mixin which specializes (directly or not) another class, this must not lead to MI: the class specialized by the mixin must be a direct or indirect superclass of the class. It means that removing mixins from the transitive closure of specialization must lead to SI.

The mixin approach is thus very close to SI and MST of JAVA. The difference is that mixins may have code and attributes, and that their specialization is quite restricted. To take up a distinction from linguistics, a class is categorematic, whereas a mixin is syncategorematic: it cannot stand alone by itself [Lalande 1926].

5.4.1 Multiple inheritance variant. There is a striking resemblance between mixins and the application of MST techniques to MI proposed by Myers [1995] (see section 5.1.4): classes are

\footnotetext{
11 According to [Bacon et al. 2002], the average attribute number in a class is small, around 4 or 5: the overhead would be $20 \%$.
} 
primary superclasses, whereas mixins are secondary superclasses. The approach by method copy applies without any adaptation, even in separate compilation: it suffices to do with mixins what $\mathrm{C}++$ does with templates, not compile them (see section 6.2). This connection between mixins and templates is not fortuitous: mixins are often presented as parameterized heir classes, i.e. as classes parameterized by the superclass of the class resulting from their instanciation. As a matter of fact, $\mathrm{C}++$ templates allow such usage [Ernst 2002]. Let $A$ be a class, $M$ a mixin: then, defining a subclass $B$ of both $A$ and $M$ is the same as defining $B$ as a subclass of $M\langle A\rangle$ [Bracha and Cook 1990; Ancona et al. 2000]. But there is no way to get an implementation from this analogy: implementation of genericity proposed in section 6.2 does not allow to make $M\langle A\rangle$ a subtype of $A$.

[Myers 1995] second variant applies also: accesses to self attributes are then optimized in the class methods and no double compilation is needed as classes are in SI. However, contrarily to SI and MST which allows a more efficient implementation than plain MI, it does not seem that mixins bring such an improvement. More generally, the fact that a class $C$ is abstract, i.e. without proper instances, does not bring any significant optimization: some data structures can be saved for the case where $C=\tau_{d}$, since $\tau_{d}$ is never an abstract class. In the case of mixins, a basic point is that concrete classes are in SI, thus allowing Invariant 2.1.2 for classes. On the whole, MI overhead is only within mixins. In some way, the mixin approach can be likened to NVI: both improve efficiency at the detriment of semantics or reusability.

5.4.2 Single subtyping variant. The section 5.2.1 schema (Figure 10) can be extended to separate compilation of mixins in the following way. The only differences with interfaces are attributes and method bodies defined in the mixins. As a mixin doesn't specialize another mixin, one can group attributes and methods proper to the mixin, in the object layout and in method tables. A mixin method needs only to know the two shifts correponding to attributes and methods for accessing self. As self is immutable, those two shifts can be computed only once, when entering the method. They also may be cached in the same way as interface method tables. As for attributes and methods inherited from a superclass, they are accessed in the same way as for a class.

The definition of mixins is rather fuzzy. The examples given by [Bracha and Cook 1990] make them orthogonal to interfaces: they are not aimed to introduce a new type but to discipline the use of call-next-method (see section 6.4.1). The implementation proposed here is based on our precise specification which forbids specialization between mixins: a unique shift is thus enough. If mixins could specialize mixins, one shift per specialized mixin would be necessary and this implementation would be no more justified.

\subsection{Evaluation}

Two main variants must be considered: à la JAVA or à la THETA. In both, time efficiency is the same as SST as long as class-typed entities are concerned. In the THETA variant, method calls to interface-typed entities is constant-time, as with MI, with simplified shifts. In the JAVA variant, the shifts are avoided but either method calls are no more constant-time, or there is an interface table flow which has to be experimented. Static space overhead is rather small, due to sharing, and dynamic space overhead is null in JAVA and small in THETA.

Both implementations have reasonable and close costs as long as interfaces are not intensively used as it is probably the case for hand-made programs. However, when interfaces are numerous, for example when they are automatically computed [Huchard and Leblanc 2000], the THETA variant could bring a too large dynamic space overhead. Moreover, the type annotations also can be automatically computed: in such a case, the interface-typed entities might be numerous and the constant-time variant may be better. A more precise comparison between those two main variants, as well as between subvariants, would need experiments.

Among the various applications to plain MI, only the approach of THETA by simulating accessors is convincing: MI overhead is reduced without semantic flaw. The only extra cost is a double compilation of each class and an easy analysis, at link-time, to choose the one to use according to the way the class is specialized. The other approaches are not convincing: method copy is incom- 
patible with separate compilation and badly compares with other global techniques (see section 7). As for mixins, they are a restriction to MI and reusability which may be not counterbalanced by efficiency increasing.

\section{COMPLEMENTS}

\subsection{Type variant overriding}

It is well known that type safety imposes that the parameter type in the overriding method must be a supertype of the one in the overridden method, whereas the overriding return type must be a subtype of the overridden one. The return type is said covariant, and the parameter type contravariant. As for attributes, they must be invariant. The fact is that strictly contravariant parameters are not interesting, since the models that one wants to implement are mostly covariant. See for example [Cook 1989; Weber 1992; Castagna 1995] for a criticize of covariance and [Meyer 1997; Ducournau 2002b] for a defence. Combined with the difficulties of implementation that we will see, this explains why languages as $\mathrm{C}++$ and JAVA forbid parameter type variance, otherwise than as static overloading. However, implementing variant overriding comes into question, either for type safe overriding, or for pure covariant languages like EIFFEL. In all cases, type variant overriding amounts to casting, either upcasts or downcasts.

6.1.1 In single subtyping. With SST, object references are invariant w.r.t. static type: thus safe overriding need no specific implementation and unsafe overriding amounts to dynamic type checks.

Covariant parameters. It may be done in the overriding callee, with the drawback that it would be systematically done, even when the static type of the parameter in the caller is a subtype of the parameter type in the callee.

In a different context (see section 5.1.3), Myers [1995] proposes to assign an offset to each method signature, not only to each method name. This looks like static overloading implementation (which is of course incompatible with type overriding) but two different offsets may here reference the same method address: offsets express syntax, whereas addresses in the entries express semantics. The various entries corresponding to the same method with different signatures and offsets point to thunks which do the required type checks. For a method $m_{C}\left(t_{1}, \ldots, t_{k}\right)$, overriding some methods $m_{C_{i}}\left(t_{1}^{i}, \ldots, t_{k}^{i}\right)$ with different signatures and offsets $\delta_{i}$, the $C$ method table contains, for each offset $\delta_{i}$, a pointer to a thunk which type checks parameters for all $j$ such that $t_{j}^{i} \neq t_{j}$, before jumping to the $m_{C}$ address.

Covariant attributes. Read accesses are as usual, at the condition that write accesses always check the assigned value. There are two ways to type check it: either one systematically uses writer methods (see section 4.4) and one reduces the question to covariant parameters, or one type checks in the caller. One needs then a dynamic downcast, since the attribute type depends on $\tau_{d}$. Thus the type identifier must be stored in the method table:

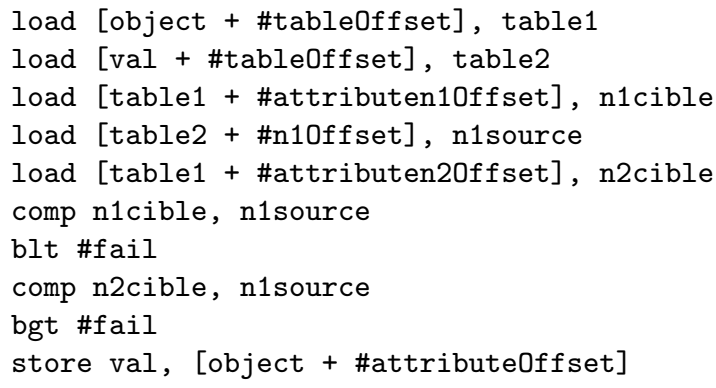

Both techniques have drawbacks. The former imposes a method call for each attribute writing, but the type check is done by a thunk, only when needed. The latter avoids a method call but requires an access to the table and a type check in any case, even when there is no overriding. Dynamic downcasts are clearly less costly than method calls plus static downcasts $(2 L+4$ vs. $4 L+B+3)$. As against this, the code sequence for dynamic downcasts is longer. 


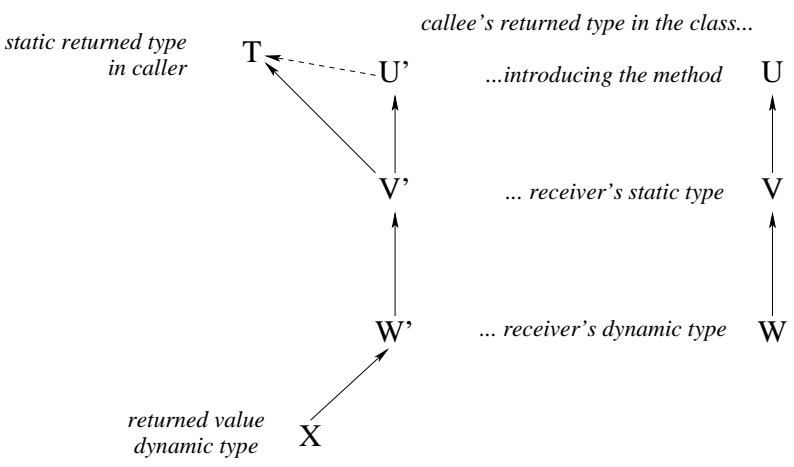

Fig. 11. Covariant return type: when $U^{\prime} \neq W^{\prime}$, upcast from $W^{\prime}$ to $T$ is dynamic whereas casting from $U^{\prime}$ to $V$ is static but no more upwards.

EIFFEL anchored types. They bring two kinds of optimizations. Firstly, all entities with the same anchor may share an entry in the method table. Secondly, some type checks may be avoided, when the compiler can prove that it is safe, typically when two anchored types are anchored to the same receiver: this is the only case where an anchored type may be its own subtype. [Meyer 1997] proposes the so-called catcall rule to ensure type safety by forbiding to access covariant properties when the receiver is polymorphic $\left(\tau_{d} \neq \tau_{s}\right)$. This rule does not work in separate compilation. Moreover, it is too restricted since it forbids calls that a global analysis might accept.

6.1.2 Multiple inheritance. Even type safe overriding is now costly because of the shifts between subobjects. $\mathrm{C}++$ allows only return type overriding ${ }^{12}$ : this may be not only because of type safety and static overloading.

Covariant return type. In multiple inheritance, covariant return type is safe but a shift is needed, which must be done either in the callee or in the caller. However, satisfying Invariant 3.1.1 is now a question: what should be the static type of the returned value? Two alternative invariants can be envisaged.

INVARIANT 6.1.1. The static type of the return value is the return type in the class which introduces the method ( $U^{\prime}$ in Figure 11).

INVARIANT 6.1.2. The static type of the return value is the return type of the callee $\left(W^{\prime}\right.$ in Figure 11).

The former has the advantage of saving a global invariance: the callee does an upcast towards $U^{\prime}$. When $U^{\prime}$ is a subtype of the type $T$ of the entity using the returned value, the caller does a second upcast. Otherwise, a downcast or sidecast is required, but only if the overriding is visible from the caller, i.e. if $U^{\prime} \neq V^{\prime}$.

With the latter invariant, the solution will consist in a thunk, which does the shift $\Delta_{W^{\prime}}^{\uparrow}\left(V^{\prime}\right)$, for each pair $(V, W)$.

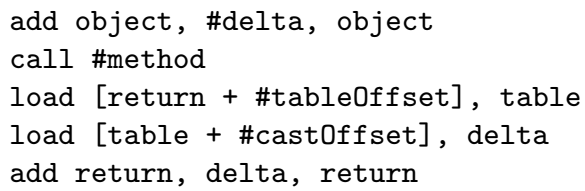

A second cast from $V^{\prime}$ to $T$ must then be made in the caller. The main advantage is that there is no more downcast, and that it does cost only when needed, i.e. for pairs $(V, W)$ such that $V^{\prime} \neq W^{\prime}$. The main drawback is that the method call in the thunk is no more terminal, i.e. it is a call, not

\footnotetext{
12 Ellis and Stroustrup [1990, pages 210 sq. and 421] say that this is part of the ANSI specification, but that it would complicate method calls. Effectively, it was not compatible with some casting prohibitions (see section 4.1). Covariant return type is however explicit in the current language specifications [Koenig 1998]. Lippman [1996] mentions it only in a NVI case.
} 


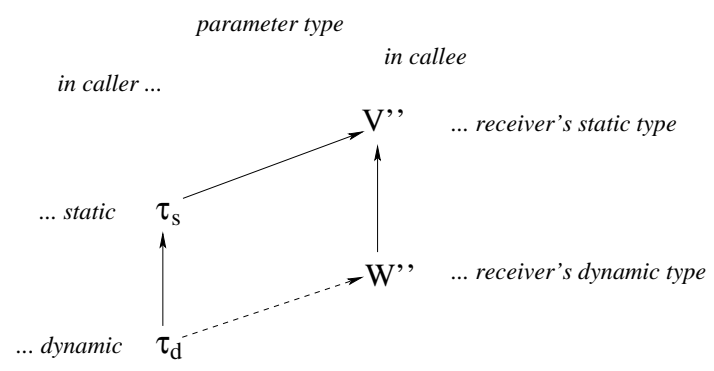

Fig. 12. Covariant parameters: a sidecast from $\tau_{s}$ to $W^{\prime \prime}$ is needed.

a jump. However, the second part of the thunk may be generated when compiling $W$, as $V$ is a superclass and the cast explicit: only the first shift on the receiver depends on $\tau_{d}$. Therefore, a solution to this double call may be to generate one function per superclass $V$, when compiling $W$, with usual thunks for jumping to these functions. This is not too expensive if either the method is small, or there are only a few $V$ for which such a thunk must be generated. When this is not the case, functions with multiple entry and exit points may avoid the double call [Lippman 1996, p. 137]. Of course, NVI will simplify this, as both shifts are static.

Variant parameters. When the parameter type is invariant, parameter passing needs only a static upcast from its static type in the caller to its static type in the callee: the shift is given by the table $\Delta_{\tau_{s}}^{\uparrow}$.

With a stricly contravariant overriding, casting is always upwards and safe, but it is no more static, since the target depends on the receiver's type. Extending method tables to parameter shifts would be possible, but very costly in static space: thunks are certainly more adapted and type overriding would not add more thunks.

The case of covariant overriding is described in Figure 12 and 6 types are implied:

$-\tau_{s}$ and $\tau_{d}$ are the static and dynamic types of the parameter in the caller;

$-V^{\prime \prime}$ and $W^{\prime \prime}$ are the static types of the parameter in the respective methods of the receiver's static and dynamic types.

By construction, 3 subtyping relationships are posed:

$$
\begin{array}{rr}
\tau_{d}<: \tau_{s} & \text { polymorphism } \\
\tau_{s}<: V^{\prime \prime} & \text { compile-time type checking } \\
W^{\prime \prime}<: V^{\prime \prime} & \text { covariant overriding }
\end{array}
$$

The goal is to check that $\tau_{d}<: W^{\prime \prime}$ and to shift with $\Delta_{\tau_{s}, W^{\prime \prime}}$ : casting may be upwards, downwards or sidewards, but it is dynamic. There are two approaches:

$$
\begin{array}{rrr}
\Delta_{\tau_{s}, W^{\prime \prime}}= & \Delta_{\tau_{s}, V^{\prime \prime}}+\Delta_{V^{\prime \prime}, W^{\prime \prime}}= & \Delta_{\tau_{s}}^{\uparrow}\left(V^{\prime \prime}\right)+\Delta_{\downarrow}^{V^{\prime \prime}}\left(W^{\prime \prime}\right) \\
\Delta_{\tau_{s}, W^{\prime \prime}}= & \Delta_{\tau_{s}, \tau_{d}}+\Delta_{\tau_{d}, W^{\prime \prime}}= & \Delta_{\Downarrow}^{\tau_{s}}+\Delta^{\Uparrow}\left(W^{\prime \prime}\right)
\end{array}
$$

In the two cases, a first static shift is done in the caller. A type check and a dynamic shift are then done, either in a thunk (11), or in the callee (12). The former approach has the advantage of imposing a casting only when needed. Notice that thunks and subobjects exempt from the signature indexing of Myers [1995].

Covariant attributes. This is a mixing of return type and parameter type. One must first choose one of the two invariants 6.1 .1 or 6.1 .2 to rule the attribute value. A read access needs then exactly the same casting in the caller as for a method call. A write access needs first a type check, done with a dynamic downcast: the target type must be stored in the method table, as in SST. With Invariant 6.1.1, an extra upcast will be needed. Implementing write access with true accessors may be a solution (see section 4.4 ), but it would be of no use for read access. 
Thunks balance. Multiple inheritance overhead notably increases - it roughly doubles - as soon as overriding is not invariant, even in the safe case of return type. However, covariant method overriding may be implemented within the thunks, without increasing their number-one thunk per pair $\left(\tau_{s}, \tau_{d}\right)$ - with the advantage that a true overhead occurs only in case of effective overriding between $\tau_{s}$ and $\tau_{d}$ (as in SST with the technique of Myers [1995], but without needing extra offsets). In this particular case, thunks gain credence. On the other hand, for attributes, thunks should be avoided as they impose true accessors and systematic extra method calls. As against this, downcasts are systematic.

On the whole, for a method introduced by a type $U$, there is exactly one thunk for each pair $\left(\tau_{s}, \tau_{d}\right)$ with $\tau_{d} \preceq \tau_{s} \preceq U$. Each thunk does successively:

- the cast on the receiver (Figure 3), from $\tau_{s}$ to the type $W$ which defines the method inherited by $\tau_{d}$;

- the possible downcasts on the parameter whose static type in $W$ is a strict subtype of the type in $U$ (Figure 12),

- the method call itself,

- a possible upcast on the return type (Figure 11).

When $\tau_{s}=W$, the thunk is the method itself, without any shift or cast.

With another MI implementation. With other techniques like NVI or compact tables, type overriding is roughly implemented in the same way. However, as tables may be shared between several static types, the technique of Myers [1995] consisting in assigning different offsets to different signatures may be an improvement.

\subsection{Genericity}

Instanciations of parameterized classes are usual classes: therefore, they are implemented as usual classes, with one or several method tables, a type identifier and the object layout is made of an attribute table plus some pointers to method tables. However, compiling parameterized classes rises specific problems. There is a simple way to elude them: no compilation at all, as $\mathrm{C}++$ does with templates. In that case, each instanciation of a parameterized class amounts to generate a new non parameterized class, where actual types have been substituted to formal types. All arguments in favour of separate compilation urge to compile parameterized classes: see, for instance, the criticize of $\mathrm{C}++$ templates by Lippman [1996]. Moreover, such a compilation allows code sharing between the different future instanciations of the same parameterized class. This is what Odersky and Wadler [1997] call a homogeneous implementation, as opposed to C ++ heterogeneous implementation.

We will mostly consider here bounded genericity, i.e. parameterized classes $A\langle T<: B\rangle$ where the formal type $T$ is constrained to be a subtype of some bound $B$ : this is the best way to allow static type checking. Match-bound polymorphism, i.e. replacing subtyping by matching for the bound [Bruce et al. 1997], has no effect on the implementation. As a consequence of contravariance rule, when $D<: C<: B$, the subtyping $A\langle D\rangle<: A\langle C\rangle$ is generally unsafe: it is type safe only when the formal type is never used in contravariant position [Cook 1989; Weber 1992].

6.2.1 Type identifiers and recursive parameterized types. Type identifiers are a first specific problem. Given a parameterized type $A\langle T<: B\rangle$, the type identifier of an instanciation $A\langle C\rangle$, may be constituted by the identifiers of $A$ and $C$. Therefore, a simple solution is to add an entry in the method tables for the type identifiers of each formal type. When the bound is itself a parameterized type, the type identifiers of the parameters' parameters must also be present, at least if one wants the subtyping $A\langle D\rangle<: A\langle C\rangle$. However, the bound may be an unparameterized supertype of some parameterized type: the number of type identifiers is not statically known. Thus, the correct scheme will be to reference the method tables of the formal types, not their identifiers.

Moreover, this will allow to implement recursive parameterized types, for instance when $A\langle T<: B\rangle<: B$ : the code of such a class may contain recursive instanciation such that $A\langle A\langle T\rangle\rangle$. Implementing the 


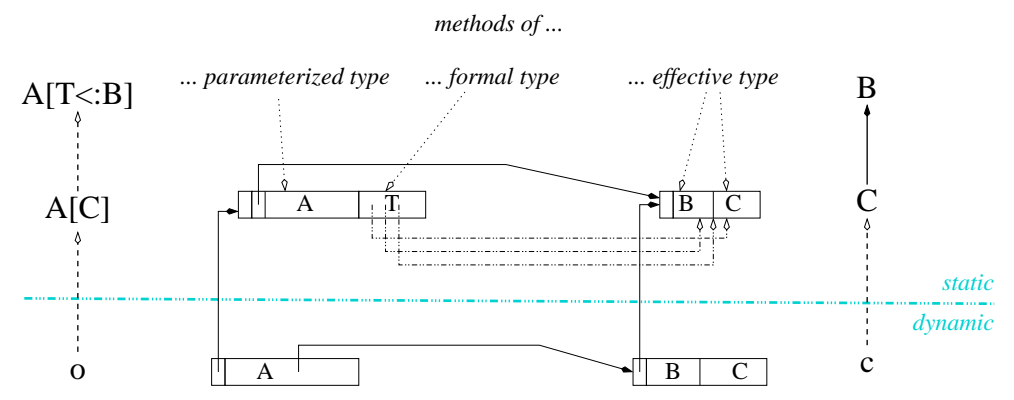

Fig. 13. Parameterized classes: method table with multiple subtyping

data structures needed for such classes cannot be done neither at compile-time nor at link-time. Therefore, if one wants to avoid copying method tables at run-time, at each instanciation, the object layout of such classes should be made of one pointer to the statically computed method table, plus one pointer to the type identifers tree, dynamically computed from the self type identifier tree. Of course, the compiler must detect such recursive cases, which may be indirect, e.g. $A\langle T<: B\rangle<: B^{\prime}$ and $A^{\prime}\left\langle T<: B^{\prime}\right\rangle<: B$. Obviously, in that recursive cases, heterogeneous implementations lead to infinite loops.

6.2.2 Instanciation with primitive types. A heterogeneous approach may be better when a formal type is instanciated by a primitive type, above all in case of collections which need a particular efficiency. In this case, a homogenous approach would indeed impose the use of wrappers, as in JAVA. So, compiling a bounded parameterized type $A\langle T<: B\rangle$ where the bound $B$ is a universal type, will amount to produce an homogeneous code shared by all non primitive classes plus a specific code for each primitive type. An alternative consists in only one implementation for all primitive types, where the method table for the formal type is a field in the parameterized class layout: for the same reason as with type identifier trees, two pointers in the object layout will avoid copying method tables at run-time. This is an usual way of implementing genericity when there is no subtyping, as in CLU [Atkinson et al. 1978; Myers and Liskov 1994]. Of course, the instanciation $A\langle B\rangle$ needs a third kind of technique, e.g. wrappers or method table flow: the subtyping $A\langle C\rangle<: A\langle B\rangle$ is possible if attributes are encapsulated.

6.2.3 In single subtyping. Invariant 2.1.2 makes bounded genericity implementation very easy: attribute and method offsets of the formal type are the same as the bound. Notice that genericity may give implicitly rise to MST as soon as one accepts subtyping between different instances of the same parameterized type (Figure 14-c/d). But this restricted case of MST does not require special implementation. The key point is Invariant 2.1.2, not SST: therefore this implementation is compatible with MI and coloring (see section 7.4).

6.2.4 In multiple subtyping. When the bound $B$ is a class, SST implementation still works. On the other hand, when the bound $B$ is an interface, offset invariance is no more ensured. A solution is extending the parameterized class method table by a conversion table converting offsets from formal type to actual types. Method calls need then one extra table access (Figure 13). A shift to method groups of each supertype may replace conversion. However, pointers to method tables is not possible, because of polymorphism. If $A\langle C\rangle$ table contains a $C$ method address, an entity typed by $C$ could not be valued by a subclass $D \prec C$. Method call is then:

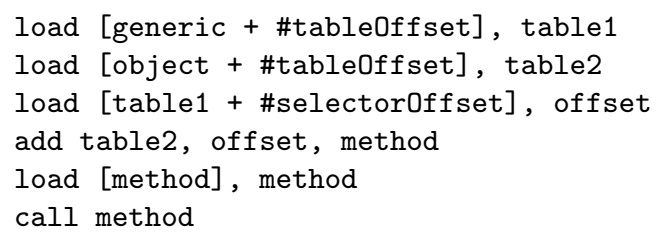

In PIZzA [Odersky and Wadler 1997], an alternative to bounded genericity is to pass methods as parameters of the instanciation. This reduces easily to the previous implementation: method 


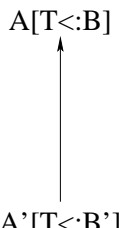

(a)

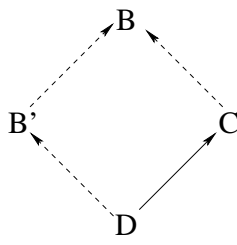

(b)

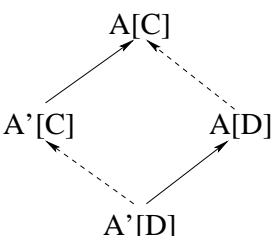

(c)

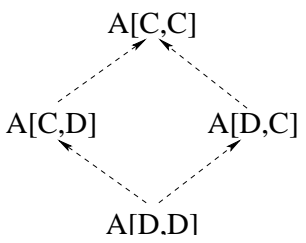

(d)

Fig. 14. Specializing parameterized classes

offsets are effectively passed as parameters and assigned to the conversion table. More generally, the presented technique amounts to consider methods of the formal types as methods of the parameterized type: but it is implemented without explicit extra method call. It is then a priori adapted to all the techniques described for MST as long as actual types are classes. When a formal type is instanciated by an interface, i.e. the bound itself is an interface, some restrictions are needed. In the MI variant (see section 5.1), accessing an interface needs a shift: all method calls will need a shift, even when the type is instanciated by a class. As for the SST variant (see section 5.2.1), it does not allow an instanciation by either a class or an interface, since method calls are realized in a different way, as explicited in the JVM by different operations, invokevirtual and invokeinterface [Meyer and Downing 1997]. So, in both cases, an efficient solution is a double compilation, according to whether the actual type is a class or an interface: this means $2^{k}$ compilations when there are $k$ interface-bound parameters.

With bounded genericity, upcasts from the formal type $T$ to the bound $B$ (or to a bound supertype) must be considered: the problem occurs only with MI variant, when $B$ is an interface. The static solution is to add an entry in the table of $A$, containing the shift from the actual type to $B$. Other casting from or to $T$ seems unsound.

Specializing parameterized classes. Two kinds of specialization are to considered (Figure 14). When $A^{\prime}\left\langle T<: B^{\prime}\right\rangle \prec A\langle T<: B\rangle^{13}$, then, following the definition, $A^{\prime}\langle C\rangle \prec A\langle C\rangle$. The proposed implementation is compatible with such a specialization: one only needs to extend the method table of $A\langle T<: B\rangle$ with methods introduced in $A^{\prime}$. Casting between $A$ and $A^{\prime}$ is as usual.

Some languages as EIfFEL accept that $A\langle D\rangle<: A\langle C\rangle$ when $D \prec C$. The unsafe cases must be handled in the same way as for unsafe covariant overriding (see section 6.1). However the implementation imposes some restriction. There is no problem when $C$ and $D$ are classes: then $A\langle C\rangle$ and $A\langle D\rangle$ share the same method table. When $C$ is an interface, the MI variant rises a problem due to the possibly different offsets for $C$ and $D$. Moreover, if $D$ is a class, SST variant also rises a problem, since method call techniques differ: a low-overhead solution is that calls to interfaces also work for classes. Thus, combining those two kinds of specialization does not lead to MI problems, since only the former add some entries in the method table.

When it is possible, the latter case of specialization makes downcast possible: casting from $A\langle C\rangle$ to $A\langle D\rangle$ or to $A^{\prime}\langle D\rangle$ is made possible by the fact that a parameterized type has pointers to its parameter type identifiers. Then, downcast from $A\langle C\rangle$ to $A^{\prime}\langle D\rangle$ imposes two type checking, first on the parameterized class, then on the parameter.

Genericity in Java. Many generic extensions of Java have been proposed: PIzza [Odersky and Wadler 1997], GENERIC JAVA [Bracha et al. 1998], NEXTGEn [Cartwright and Steele 1998] and many other [Agesen et al. 1997; Solorzano and Alagić 1998]. None of them takes the approach presented here because it is not compatible with JVM: compatibility would imply to realize method calls to formal types with an explicit call to self, whereas we only simulate this call with an offset. In practice, homogeneous propositions replace the formal type with the bound (type erasure) and add safe downcasts which need no implementation in the SST variant.

6.2.5 In multiple inheritance. Multiple inheritance of parameterized classes is possible: so they must be implemented as non parameterized classes (section 3). In each method table, method calls

\footnotetext{
$\overline{13}$ This means that $A^{\prime}\langle T\rangle \prec A\langle T\rangle$, for all $T<: B^{\prime}$, with $B^{\prime}<: B$. 
to formal types are the same as in MST. On the other hand, attributes cannot use neither SST technique for attributes, nor MST for methods: they are indeed invariant only w.r.t. the static type which introduces them. An attribute $p$ accessed on the formal type $T$ is necessarily known by the bound $B$, thus $B \preceq T_{p}$ and $\delta_{p}$ is known and invariant (see page 9 ). However, the upcast from $T$ to $T_{p}$ is not static as $T$ is formal. Thus, for each $T_{p}$, an entry of the method table must contain the position of the shift $\Delta_{T, T_{p}}$ in the $\Delta_{T}^{\uparrow}$ table, i.e. the value of $i_{T}\left(T_{p}\right)$.

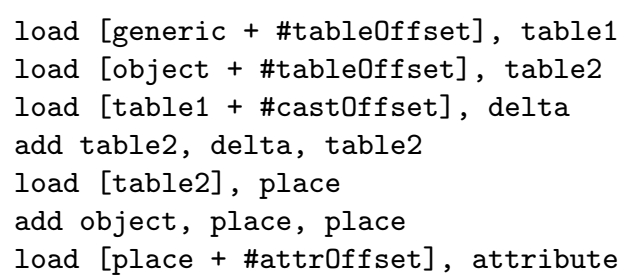

Upcasts from $T$ to $B$ need also to add in the method table of $A$ an entry containing the value of $i_{T}(B)$ : one may generalize by adding an entry for each supertype fo $B$.

However, as in MST with the MI variant, subobject implementation makes the subtyping $A\langle D\rangle<: A\langle C\rangle$ difficult: entities typed by $C$ and $D$ don't point on the same subobject. Thus substitution is not possible. In fact, the problem is exactly the same as for variant overriding of attribute and return types (invariants 6.1 .1 or 6.1.2). For a type safe language, this is a minor limitation as this specialization is rarely safe [Day et al. 1995]. For a covariant language as EIFFEL, this is more problematic: systematic casting is needed (see section 6.1) and the method tables of $A\langle D\rangle$ and $A\langle C\rangle$ are no more shared.

\subsection{Shared class attributes}

The term of class attributes is fuzzy and at least three different notions are concerned:

$-\mathrm{C}++$ and JAVA static variables are not relevant to object orientation as they cannot be selected, in case of overriding, according to the dynamic type of an object;

-attributes of classes considered as instances of meta-classes, in a reflexive model, à la SMALLTALK or Clos: this notion has no direct equivalent in the languages that we consider here;

- the fact that an ordinary attribute could be shared by all instances of a class: it may then be allocated in the class data structure instead of in every instance: this is the meaning of the keyword : allocation : class in CLOs: to avoid ambiguity we will call them shared attributes.

The two last notions differ by their semantics: we can illustrate the former by the set of proper instances of a class, which is a property of the class, not of the instances, and the latter by the side number of polygons, which is 4 for all instances of quadrilateral, and 3 for all instances of triangle. The difference is that a class does not share its instance set with another class, whereas all subclasses of quadrilateral share the same side number, except odd ones as 3.5-side-quadrilateral...

Shared attributes will be implemented in the method table: as several classes, with different tables, may share the same attribute, an extra indirection by a wrapper is needed when the attribute is mutable. The wrapper is needless when the attribute is read-only. Multiple inheritance does not complicate the case.

Curiously enough, this sound and efficient mechanism is not proposed by commonly used languages: $\mathrm{C}++$ and JAVA offer only static variables. In EIFFEL, constant features amount to read-only ${ }^{14}$ class attributes: once features are a variant where the constant is itialized by a first call, subsequent calls returning the same value. When they are frozen, this is an equivalent of static in $\mathrm{C}++$ and JAVA. The keyword :allocation of Clos has actually a more complicated specification than ours, as it may take two values, : instance or : class, and it may be overridden. This is against all attribute invariants, whether they are in single or multiple inheritance. Plain accessors (see section 4.4) is a way to implement it in a general way, with a dynamic space saving,

\footnotetext{
14 The binding between the name and the value is immutable, not the value itself.
} 
but at the detriment of time efficiency. An alternative would be to implement shared attributes in the instances, with a wrapper to ensure value sharing, thus at the detriment of time. On the whole, the complete specification of the keyword should be reserved to attributes explicitly declared at introduction.

\subsection{Calling super}

Almost all languages offer a mechanism for calling the overridden method from the overriding method: this is a sound way to save a kind of behaviour invariance in spite of overriding. In SMALLTALK and JAVA, this is realized by the keyword super - a pseudo-variable as self - which consists in calling the superclass method on the current receiver, i.e. the self value. Although this mechanism is designed for calling the overridden method, super syntactically requires the method name: in fact, it allows to call any superclass method ${ }^{15}$. In SI, the superclass method is unambiguously determined as well as the method offset: everything is invariant w.r.t. dynamic types, and the super call is static.

6.4.1 In multiple inheritance. Super does not work since the superclass method is not uniquely determined, in the general case. However, there exists at least three variants of the mechanism: static call (::) in C++, precursor in EIFFEL and call-next-method in CLOs.

The $\mathrm{C}++$ operator $::$ is more general than super as it allows to call any method as a classic static procedure call: the technique is the same as for a method call, except that an access to method table may be needed for the shift, not for the address. The mechanism is quite versatile, but it has an important drawback, liken to repeated inheritance (see section 4.1): in the diamond example of Figure 2, when the method $m$ in class $D$ calls methods $B:: m$ and $C:: m$, which both call $A:: m$, then the latter is executed twice when calling $D:: m$.

In EIFFEL, precursor differs from super in JAVA and SMALLTALK on two points: first, the mechanism applies to MI but only when there is no multiple inheritance conflict, second it applies only on the current method name, which corrects the super flaw. As the overridden method is unambiguously determined, implementation is the same as for :: in $\mathrm{C}++$.

CLOS call-next-method is more original: it consists in calling the next method in the linearization order of the superclasses of the receiver's dynamic type [Ducournau et al. 1994]. The main advantage is to avoid the repeated inheritance problem. An important drawback is that the next method will depend on the dynamic type, not only on the static type. In the diamond example, the linearization of $D$ (resp. $B$ ) may be $\{D, B, C, A\}$ (resp. $\{B, A\})$ : so, in $B$, the next method will be in $C$ (resp. $A$ ). This causes some trouble to modularity and it makes implementation more difficult. A direct static call does not work. A simple solution is to assign an extra offset in the method tables, for each method. Whereas primary method entries contain the same address for all static type of the same dynamic type (Figure II), call-next-method entries will contain different addresses. Those extra offsets are needed only for methods which explicitly use call-next-method, and only in the method tables of the static type where the method is defined: thus this new mechanism would cost only when and where it is used.

6.4.2 The case of BetA. The BETA language [Kristensen et al. 1987] differs from all other object-oriented languages by the way method combination is realized. Instead of calling the overridden method from the overriding method as with super, the overridden method calls the overriding method with the keyword inner. So there is neither true overriding, nor late binding for method calls which are always static: method tables are only needed for inner calls. Nevertheless, all the techniques reviewed here more or less apply.

\section{$6.5 \mathrm{Null}$ pointers}

Any variable or attribute typed by a class must be explictly initialized. An intraprocedural data flow analysis may detect uninitialized local variables, but this is more difficult for attributes, which may be initialized some time after creation. Thus, an initialization with a null pointer is a way to

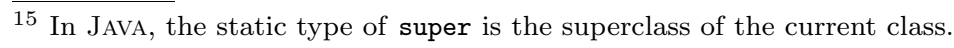


avoid random memory state. The simplest solution consists in a distinguished value null and in checking at every object access (method call, attribute access, cast) that the receiver is not null.

Even when those checks are restricted to accesses which are not proved to be non null, this would add a considerable overhead. There are two general kinds of alternatives. The first one depends on hardware and operating system: for instance, in [Alpern et al. 1999] null has address 0, and all offsets are negative as, in the AIX system, negative addresses rise an interrupt. Software based solutions consist in a distinguished instance null for each class: all its attributes are initialized to null and its method table points to methods which signal an exception. This object must be allocated in the code area, assumed to be read only, in order to prevent any assignment. However, read accesses are possible, propagating null values, which may make debugging difficult.

Different null objects may be shared: in SST, a unique null object is even possible if the method table structure allows it (method tables may contain more information than method addresses). This object will be constituted by the largest object layout, with the largest method table. In MI, a unique object is also possible: it will consists in the unique instance of the class $\perp$, subclass of all classes, and will be constituted by one subobject by class. Of course, those shared null objects must be computed at link-time (see section 7).

\subsection{Garbage collection}

Automatic memory handling is an argument for fiability: this has been well understood by designers of Lisp, Smalltalk, Eiffel and JaVA. There are many garbage collection (GC) techniques. All need some information concerning individual objects: length of memory area, boolean status of the object and possibly a pointer on a new generation. Those informations may be contained in the object layout or implemented in separate tables. We will only consider the former case. [Wilson 1992; Jones and Lins 1996] are reviews of garbage collection techniques.

6.6.1 Single subtyping. Standard SST implementation is well adapted to GC: all proper instances of a class have the same length, so it may be stored in method tables. Moreover, reference invariant avoids pointing inside memory areas.

A few marking bits are needed for different status informations during the GC process: they are partly used for marking living objects, reachable from memory roots, and are proper to each instance. With static typing, there is no way to dynamically distinguish an immediate value from an address. Thus, an easy way to search for all living objects consists in defining a specific method for this purpose, which is automatically generated by the compiler [Colnet et al. 1998]. When marking is not implemented in this differentiated way, it must conservatively assume that each field in the object layout is a potential pointer on a living object, which is slower: the GC is then called conservative [Boehm 1993]. However, GC must be at least semi-conservative since root extraction from registers and stack requires such an assumption. In all generality, checking that a memory word is a pointer to an object may consist in checking firstly that this is a valid address, secondly that the first field is a pointer to a method table, which is easier since method tables are static. This is a necessary condition but it is not sufficient. Now calling the marking method on an address which is not an object may lead to unexpected results: thus, when marking is implemented by a method, root extraction must be exact and it requires a more precise condition. An alternative technique will use a mapping of the heap into a bitstring: each bit represents a word (resp. double-word) allocation, causing a $3 \%$ (resp. $1.5 \%$ ) memory overhead. Allocating objects of different sizes or types in different areas is also a technique [Colnet et al. 1998] which allows to reserve only one bit per object but it increases memory fragmentation.

When they are implemented in the object layout, those bits can occupy a field by itself, but this is a considerable overhead. Bit-stealing is a general way to avoid the overhead, by implementing them as low or high weigth bits of some field in the object layout. For instance, when the object size is not shared, it needs only two bytes and one extra byte is free for marking bits. In the general case, one can use either the pointer to method table, or the first attribute, for instance when it is a pointer. In both cases, there is a uniform overhead, with two more instructions and cycles for removing those extra bits, at each access to the field. [Bacon et al. 2002] present some experimental statistics on this approach in JAVA, where pointer to method is used. However, the 
overhead might be null if the value of those bits is unchanged between two accesses to the field: only the methods implementing the GC, or the access to the first attribute, should be compiled in a specific way to remove the extra bits which are no more at their default value. So the overhead depends on whether the GC is implemented as a concurrent background task, or as a blocking task. Using the first attribute field supposes reserving it for an attribute which don't use 32 bits, for instance when it is a pointer. The overhead will then be supported only by one attribute. However, some classes may have only 32 bits attributes (e.g. integer): GC would then have a dynamic space overhead.

6.6.2 Multiple inheritance. When Invariant 2.1.1 is not verified, the marking stage is complicated since object references may point inside memory areas. Marking methods may help to solve the problem but a general alternative exists: each method table may have an entry which contains a shift towards the first subobject in the object layout. Moreover, this entry may be the same as for $\Delta_{\Downarrow}^{\tau_{s}}$, if one places always the dynamic type subobject first. In any case, an extra overhead must be expected. Notice that VBPTRs (see section 4.3) avoid this complication since the object layout is made of explicitly linked subobjects. In any case, GC complexity is a function of subobject number not of object number.

\section{COMPARING WITH GLOBAL COMPILATION}

Separate compilation is a good answer to modularity requirements of software engineering: it brings speed of compilation and recompilation together with locality of errors and protects source code from both infringement and hazardous modifications. With separate compilation, the code generated for a program unit, here a class, is correct for all correct future uses. Run-time generation ends with a final step linking all program units.

\subsection{Advantages of global compilation}

7.1.1 Hierarchy closure. The first advantage of global compilation is that the class hierarchy is closed: no extra class can be added after the compilation, unless a new complete compilation of the whole hierarchy. It is then possible to know if a class is specialized in single or multiple inheritance, if two unrelated classes have a common subclass, and so on. Moreover, the schema of each class is known. So, in a dual way, one knows where methods are defined: methods with a unique definition may then be treated as classic procedural calls (monomorphic calls). This is known as the unique name heuristics [Calder and Grunwald 1994]: thought its simplicity, its effect is not small as this applies to almost $45 \%$ methods in SMALLTALK.

With static typing. Hierarchy closure brings even more information: a call is monomorphic as soon as the method is not overridden in the subclasses of the static type. Moreover, a hierarchy analysis allows to determine which classes must be compiled as virtual, and which ones don't need it [Eckel and Gil 2000] (see section 4.1). Alternatively, one can decide of primary and secondary superclasses (see section 5.1.4).

7.1.2 Knowledge of method code. A second advantage is the knowledge of the code of all methods: when compiling a method, one also knows how the method is used, and when compiling a method call, one also knows the method codes for all possible callees. Many optimizations proceed from this knowledge but they all suppose an underlying general implementation technique.

\subsection{Global compilation in dynamic typing}

In a dynamically typed language as SMALLTALK, the lack of type annotations makes separate compilation quite unefficient. So, many techniques of global compilation have been worked out in the framework of dynamic typing: of course, they all apply to static typing as well.

7.2.1 Dynamic typing and single inheritance. Notice first that, with dynamic typing, SI is no more a simplification, due to definition overloading (see note 4, page 5): when the same method name is introduced in two unrelated classes, Invariant 2.1.2 is not verified, at least in separate compilation. This concerns also attributes as soon as they are not encapsulated as in SMALLTALK. JAVA type system may be understood as the minimal type system required for statically typing RR LIRMM 2002-174 
SMALLTALK: the interface notion is the way to deal with definition overloading. So, with dynamic typing, implementation is at least as difficult as with MST, or even with plain MI when attributes are not encapsulated.

7.2.2 Compilation techniques. If one puts aside dynamic search (lookup) in the class hierarchy, method call techniques are either with constant-time direct access tables or with a group of techniques summarized under the name of type prediction.

Compacted large table. Table-based techniques consist in compacting the large array obtained by a global and injective numbering of all classes and methods. As the class number (resp. method number) may reach and even exceed 1000 (resp. 10000), this table is huge, several M-entries, and too large to be implemented as such. However, the number of valid class-method pairs is far smaller, at most $5 \%$ : it is the total size of method tables in SST, $\Sigma_{C} M_{C}$. Two techniques for compacting that table have been proposed: row displacement proposed by Driesen and Hölzle [1995] and method coloring proposed and studied by Dixon et al. [1989], Pugh and Weddel [1990], André and Royer [1992] and Ducournau $[1991 ; 1997]$. The latter technique will be detailed further.

In both cases, the result is a table each entry of which either contains a unique class-method pair or is empty. However, the lack of static type checking yields that a method may be called on a receiver of a wrong type, which may amount to either an empty entry or a class-selector pair with a different selector: thus a dynamic type checking is needed but it reduces to a simple equality test between the expected method and the effective one. Static typing makes this extra test useless. On the whole, there are few empty entries, less than $10 \%$ in most of the benchmarks, with a $50 \%$ upper bound for all experiments: compared to standard MI, the total size remains close to $\Sigma_{C} M_{C}$ (see appendix A).

Type prediction. Type prediction originates in the idea that a given type may be considered as more likely for some method call: for instance, type integer for the method + . So, type prediction consists in compiling a method call by a comparison between the expected type and the effective receiver's type: if the test succeeds, a static call is done, otherwise a call to another technique is done. There are many variations on this basic idea. The expected type may be the receiver's type for the previous call, either on the same call site (inline cache) or globally (global cache). Prediction may also be polymorphic when the receiver's type is tested against several types [Hölzle et al. 1991]: in that case, the call sequence is a small decision tree which compare the receiver's type with all the expected types.

The technique is not a priori sufficient since cache defaults must be handled when the receiver's type is not amongst predicted types. Therefore some underlying technique is needed, for instance a dynamic lookup à la Smalltalk. An alternative is to rule out cache defaults: the decision tree must then exhaust all possible types. This is easier with static typing as the static type is available. A type analysis may give a more precise information, the concrete type. At last, it is better to balance the tree, as the number of expected types may exceed 100 [Zendra et al. 1997].

The entire call sequence is made of conditional branching: [Driesen et al. 1995] shows that they are statistically very well predicted by modern processors. Therefore, indirect branching $(B$ latency) and memory accesses ( $L$ latency) are avoided. As against this, the number $k$ of expected types, thus the branch number, may be large and the search is in $O\left(\log _{2}(k)\right)$ : but a predicted branch has a 1-cycle cost, whereas an unpredicted one has a $B$-cycle cost.

Subtyping test. In tree-based techniques, the comparison between the effective type and the expected type may be either an equality test or a subtyping test. With the former, all types must be exhausted, whereas only methods need to be, in the latter. But subtyping test is expensive in the general case. [Queinnec 1997] handles only SI and uses the coloring technique of [Cohen 1991], which is prefered to Schubert's numbering as it is incremental. Interval containment [Muthukrishnan and Muller 1996] uses Schubert's numbering. Type prediction techniques use equality tests.

Mixed techniques. There are many ways to mix table-based and tree-based techniques, by putting either tables in tree leaves [Queinnec 1997] or tree roots in table entries [Vitek and Horspool 1994]. 
Type slicing [Zibin and Gil 2002] is a generalization of Schubert's numbering and interval containment to MI. The main effect of those techniques is to save static memory w.r.t. standard tablebased techniques: the size of static data structures may be quite smaller than $\Sigma_{C} M_{C}$. However, this is detrimental to time efficiency: as with type prediction, method call is no more constant-time. Moreover, unlike pure type prediction, a table access is required.

Attributes. The case of attributes is rarely treated in the literature. It may be explained by the fact that they are often encapsulated in the methods, as in SMALLTALK: so they are accessed only on self, which has the uncommon feature to be the only statically typed entity: Invariant 2.1.2 is thus verified for them, in case of SI. Accessor simulation is sufficient for MI but non encapsulated attributes require plain accessors, because of definition overloading.

\subsection{Global optimizations}

7.3.1 Method copy. When the source code of superclass methods is known at compile-time, it is possible to copy in a class the code of inherited and not overridden methods. Method copy has been already seen as an application to MI of the THETA technique (see section 5.1.4). The main advantage of this technique, termed customisation by [Chambers and Ungar 1989], is that self becomes monomorphic: method calls to self, which are quite numerous, can be compiled by a static call, without extra memory access. When the attributes are encapsulated as in SMALLTALK, attribute invariance does not matter and attribute offsets are always static. Otherwise, non self attribute accesses must be encapsulated by accessors generated or simulated by the compiler (see section 4.4). Calls to super or static calls must be inlined. Moreover, all methods which apply only to self - either by following some specific visibility keyword, or as deduced from a global analysis - may be removed from method tables. Finally, method copy gives more precise type informations, in case of type overriding, either safe as return type, or unsafe as parameter or attribute types. Anchored types may be replaced by constant types. However, only accesses to self are optimized. An implementation technique is required for the general case. Time efficiency is improved at the detriment of static space: method code is duplicated with a factor linear w.r.t. class number. Thus this technique cannot be envisaged without an association with optimizations reducing drastically the size of the generated code. Obviously, dead code must be ruled out.

7.3.2 Automatic devirtualization. It is a common opinion that repeated inheritance is an abomination and that the keyword virtual is justified only by efficiency. A static global analysis can determine which classes must be implemented as virtual, and which classes need not [Gil and Sweeney 1999; Eckel and Gil 2000]. More precisely, it can determine how to share subobjects. On the diamond example, it is possible to merge, on one hand, the subobjects $D$ and $B$, on the other hand, the subobjects $A$ and $C$, saving 3 method tables in Table II. Devirtualization consists in partioning $\prec_{\mathrm{d}}$ into two relationships, $\prec_{\mathrm{v}}$ and $\prec_{\mathrm{nv}}$, in such a way that the latter induces AI. A simple devirtualization scheme consists in marking as virtual all classes which have two direct subclasses with a common subclass, i.e. all $A$ such that $\exists B, C, D: C \neq B, D \prec B \prec_{\mathrm{d}} A$ and $D \prec C \prec_{\mathrm{d}} A$. In a second step, one identifies virtual edges $\left(\prec_{\mathrm{v}}\right)$, in such a way that for each pair of related classes $D \prec A$, there is at most one non virtual edge towards $A$ on all paths from $D$. Of course, the choice of the only non virtual edge is a matter of optimization.

7.3.3 Type analysis. The main objective of type analysis is to determine the concrete type of each program expression, i.e. the set of dynamic types that the expression will take for all possible executions. Without loss of generality, the problem is exponential - even undecidable as an exact answer poses the problem of program termination - but simplifying assumptions make it polynomial [Gil and Itai 1998]. Type analysis may be based on the construction of a call graph, but, with object-oriented languages, the two problems are in circular dependency: a call graph is needed to get precise concrete types, but a precise call graph requires concrete types, at least for receivers. As sound type analyses compute always an approximate (upper bound) of exact concrete types, they may be more or less precise and costly. [Grove and Chambers 2001] makes a review of different techniques. A classic compromise is Rapid Type Analysis (RTA) [Bacon and Sweeney 1996]. 
A secondary goal of type analysis is to type check programs, in case of downcasts or of unsafe type overriding, and it may save some dynamic type checks [Wang and Smith 2001].

7.3.4 Dead code. An interesting by-product of type analysis is the ability to distinguish living and dead classes and code. Indeed, the call graph associated to a type analysis makes explicit the classes which are never instanciated and the methods which are never called, in that they are unreachable from the main procedure. Type analysis is thus a good way to reduce the code size of applications. However, not all applications will benefit from it. This is not the case, for instance, of applications where class instanciation results from an interaction with a user, a DBMS, another program or a network. In such a context, all application classes are alive, as well as all their methods which can be activated in the same way.

7.3.5 Inlining. This is a common optimization of procedural languages: it consists in copying the code of a callee in the caller, for instance when the call is monomorphic and the method is small or not often called. In all cases, this is a final optimization of monomorphic calls and, in global compilation, it can also be applied to type prediction. Attribute inlining must also be considered: it consists in replacing an object address by the object itself in the layout of another object, as with the EIFFEL expanded keyword. It will save a load instruction but it is only sound at two conditions: type analysis must prove that the attribute is monomorphic and alias analysis must prove that the same object will not be inlined in two different objects.

7.3.6 Small Eiffel. The GNU Eiffel compiler is typical of the use of those global techniques in the framework of a statically typed language. It is based on a double a priori: global compilation without method tables. In the object layout, the pointer to method table is replaced by the class identifier. The compilers uses the following techniques:

(1) method copy which makes self monomorphic (customization);

(2) type analysis following the RTA algorithm, which determines concrete types of all expressions;

(3) dead code and classes are then ruled out;

(4) method calls still polymorphic after steps 1 and 2 are implemented by a small decision tree, based on equality tests on type identifiers; the same technique is used for polymorphic accesses to attributes, when the offset vary according to concrete types, as well as for downcasts;

(5) finally, inlining is done in many cases.

Recompilation speed is ensured by producing $\mathrm{C}$ code. The compilation of living code from EIfFel to $\mathrm{C}$ is systematic, but $\mathrm{C}$ files are recompiled only when they have been modified. [Zendra et al. 1997; Collin et al. 1997] describe in detail those techniques and experimental results which show a clear improvement w.r.t. existing EIfFEL compilers.

\subsection{Coloring heuristics}

We detail now the coloring approach as it is quite versatile and it naturally extends to MI the SST implementation. Method coloring has been proposed by Dixon et al. [1989], under the name of selector coloring. One of the first experimentations, by André and Royer [1992], concluded to large coloring computation time and the technique was considered as uneffective ever since. However, a previous work by Pugh and Weddel [1990] had reported positive results, confirmed later by Ducournau [1991; 1997]. One may define coloring as upholding SST invariants 2.1.1 and 2.1.2 in MI.

INVARIANT 7.4.1. An attribute (resp. method) has an offset (color) invariant by specialization. Two attributes (resp. methods) with the same color don't belong to the same class.

As a corollary, two classes which have two different attributes (resp. methods) with the same color cannot be specialized by a common subclass. An injective numbering of attributes (resp. methods) verifies the invariant: therefore, an optimization is needed. The first proposition, by Dixon et al. [1989], André and Royer [1992], was to minimize the color number, which is an NP-hard graph coloring problem [Garey and Johnson 1979]. A first improvement, proposed by Pugh and Weddel [1990] and Ducournau [1991; 1997], consists in minimizing the total size of 


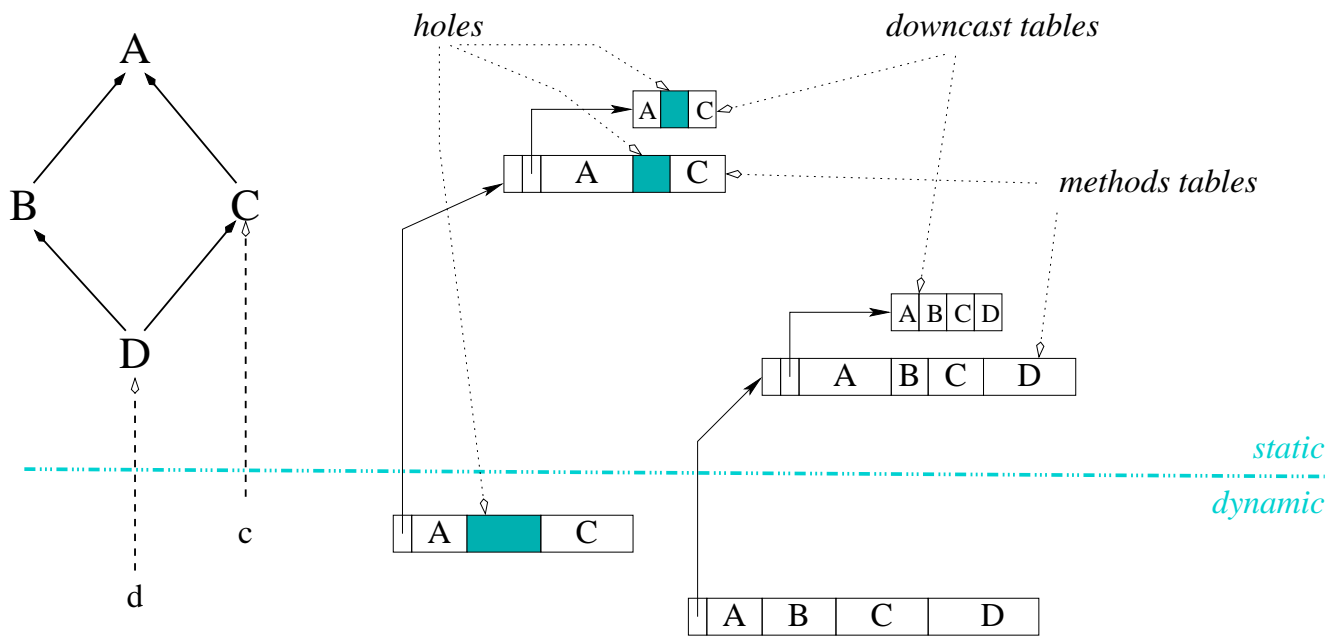

Fig. 15. Unidirectional coloring heuristics applied to classes, methods and attributes.

Table IV. Coloring bibliography

\begin{tabular}{ll|ccc|ccc|c}
\hline \multicolumn{2}{c|}{ paper } & & \multicolumn{3}{c|}{ optimization } & \multicolumn{3}{c|}{ entities } \\
acolors & size & birect. & method & attribute & class & holes \\
\hline Dixon et al. & {$[1989]$} & $\times$ & & & $\times$ & & & \\
Pugh and Weddel & {$[1990]$} & & & $\times$ & & $\times$ & & 6 \\
Ducournau & {$[1991]$} & & $\times$ & & & $\times$ & & \\
André and Royer & {$[1992]$} & $\times$ & & & $\times$ & & & \\
Ducournau & {$[1997]$} & & $\times$ & & $\times$ & & & 6 \\
Vitek et al & {$[1997]$} & & $\times$ & & & & $\times$ & \\
Ducournau & {$[2002 \mathrm{a}]$} & & & $\times$ & & & $\times$ & 45 \\
\hline
\end{tabular}

method (resp. attributes) tables: the tables resulting from coloring are then similar to SST tables, except that they may contain holes, i.e. empty entries. A second improvement, due to Pugh and Weddel [1990], is a bidirectional coloring, with positive and negative colors. The complexity of those new problems is not known but they are likely as difficult as the original problem. In any case, heuristics are needed and some experiments, by Pugh and Weddel [1990] and Ducournau [1997; 2002a], show their efficiency and that improvements are effective. Dixon et al. [1989], André and Royer [1992] applied first coloring to methods, whereas Pugh and Weddel [1990], and Ducournau [1991] applied it to attributes, and Vitek et al. [1997] and Ducournau [2002a] to classes, as a generalization of the SST technique of Cohen [1991]:

INVARIANT 7.4.2. Each class has an offset (color). Two classes with the same offset have no common subclass.

The time overhead of multiple inheritance vanishes but holes induce a small space overhead. Pugh and Weddel [1990] report a $6 \%$ hole rate on a FLAVORS program with 563 classes and 2245 attributes. Ducournau [1997] reports the same hole rate on a SMALLTALK distribution with 698 classes and 4518 methods. However, class coloring experiment on large MI programs as Geode or Lov reports a $45 \%$ hole rate, whereas the average hole rate is around $12 \%$ [Ducournau 2002a]. For static tables (methods and classes), this overhead is insignificant compared to the cubic table size of standard implementation (see appendix A).

On the other hand, for dynamic memory, the overhead may be significant: one should minimize the total dynamic memory, which needs a profiling of class instanciation. A conservative solution will be to simulate accessors (see section 4.4) instead of coloring attributes: the offset of each subobject will be colored in the same way as methods. Dynamic memory overhead disappears, at the detriment of a constant time overhead for attribute accesses: it may be reduced by Myers' [1995] double compilation. 
On the whole, coloring gives exactly the same implementation as standard SST implementation in case of SST: this corrects the main drawback of standard MI implementation. In case of MI, the overhead w.r.t. SST concerns only static tables and accesses to some attributes: nevertheless this overhead remains far from all other MI implementations overhead (sections 3 and 4). Notice that row displacement [Driesen and Hölzle 1995] would give similar efficiency for method tables and subobject offsets: however, class coloring does not seem to have any equivalent.

\subsection{Global techniques in separate compilation}

Separate compilation is not incompatible with global techniques which can be applied at link or load-time. Moreover, almost all implementations require a minimal global step, for instance for generating class identifiers by a global numbering.

7.5.1 Double compilation. The technique proposed by Myers [1995] implies a small global analysis to determine whether a class is always specialized as a primary one, or not. Double compilation can be based on other criteria, e.g. devirtualization, as long as they are invariant by specialization. It is only interesting for optimizing accesses to self and to other entities typed by the current class.

7.5.2 Link-time computation of method tables and object layout. One can distinguish two tasks in compilation. On one hand, it generates the method code. On the other hand, it generates the data structures associated to classes, together with object layouts, by fixing the size of all tables and the offset of all entities. Therefore, a general approach is to place the second task, either partly or entirely, at link-time: linking will then have to substitute in the code the values of all the offsets computed after the compilation. For instance, this must be done for Schubert's numbering (2) if one wants a truly static cast, i.e. that $n_{1}$ and $n_{2}$ values are immediate values in the code. Of course, the code generated by separate compilation must be independant of this global step: in case of optimization, the worst case must always be foreseen.

Link-time coloring. Coloring is a global technique but, as already noticed by Pugh and Weddel [1990], it could be computed at link-time: effectively, coloring requires only the schema of classes, not their code, and the generated code depends only on individual colors associated to the different entities. Those colors are small integers which could be run-time constants computed at link-time. However the approach does not seem to have been experimented.

Link-time devirtualization. Using a global technique at link-time, after a separate compilation, must be carefully evaluated. Automatic devirtualization is a good example. It may reduce the number of subobjects, thus the need to shift object references, but there is no way to know, at compile-time, whether a shift will be needed or not. Thus, the generated code will be the same as with standard MI implementation, with the only difference that, at run-time, most shifts would be null. Therefore, the only gain will be on the size of the tables, either static or dynamic: in case of SST, the layout will be the SST one (augmented with upcast tables) but the code will be the MI one. However, upcast tables also can be saved if $i_{\tau_{s}}(C)$ are computed at link-time. Therefore, link-time devirtualization could be only envisaged as an optimization for VBPTRs implementation (see section 4.3), but it is likely that it will badly compare with link-time coloring. A double compilation - according to whether all superclasses are reachable through non virtual edges - could however improve the code.

The case of empty subobject optimization is quite different as both kinds of merging can be fixed at compile-time: no link-time processing is needed. At link-time, one could only fix that some second kind merging is possible in all subclasses, but it would have no effect neither on the code nor on the layout.

7.5.3 Separate type analysis. Type analysis requires source code, but it may split up into two phases: intra-class analysis and inter-class analysis. This is an object-oriented formulation of classic intra-procedural and inter-procedural analyses. Privat [2002] proposes to joint those two phases by a template, produced by an intra-class analysis during a separate compilation and which stands for an abstract of the class code, expliciting the flow of types in the methods. At link-time, 
the global inter-class analysis will use those templates to construct the call graph and determine concrete types for all expressions in the template. Such an approach would allow to detect dead code and to rule it out from the executable. However, local optimizations as monomorphic calls and inlining are not directly possible but an optimized version could use in separate compilation the result of the previous global analysis.

\section{CONCLUSION}

On the one hand, separate compilation of SST is simple and as efficient as possible: indirect method calls are a true overhead which could be only reduced with global compilation or by an increasing of processors' capabilities for indirect branching prediction [Driesen 1999]. But SST expressivity if far from what programmers expect. On the other hand, separate compilation of plain MI presents a significant overhead w.r.t. SST: the main drawback of the standard implementation is that it is as costly when one does not use MI. Therefore, this is not surprising that recent efforts have focused on SI but MST languages, as JAVA or C\#: this is a sound middle point between the two extremes, especially if compared to $\mathrm{C}++$ NVI or mixins.

There is some evidence that standard MI implementation might be improved with the empty subobjects optimization, when it is not incompatible with VBPTRs, in $\mathrm{G}++$ for instance. However, standard implementation is not the only approach to MI. Variants such as the THETA approach correct partially the main drawback of standard implementation but they don't seem to have been effectively used. SMALl EIfFel proved that quite different approaches are possible but, in that case, definitively incompatible with separate compilation. Global compilation is obviously the way to obtain the best efficiency but its drawbacks are numerous. Therefore, a general way to improve separate compilation is to introduce a touch of global techniques in the run-time production line. This was already the case with THETA. The coloring approach combined with type analysis is an appealing idea on paper: the run-time produced using those techniques would be even more efficient than with SST implementation, due to dead code detection. However the efficiency of the link-time global step must be established in practice: we are currently working towards a prototype.

Nevertheless, global techniques have significant drawbacks. Being global, they are incompatible with library sharing between different applications, which is a common optimization of operating systems: hence, the best MI implementation allowing sharing seems to be standard MI implementation with empty subobject optimization. Moreover, besides being global, most of them are not incremental and could not satisfy the specifications of abstract machines as JVM or CLR which are mainly dedicated to mobile code.

Commonly used statically typed object-oriented languages - i.e. C++, EIfFel and JAVA - are quite perfectible: valuable suggestions include shared class attributes instead of static variables, method combination, or self encapsulation, besides private and protected keywords. The cost of implementing those features would not increase the overhead of object-orientation. This is not the case of type overriding which add some overhead especially with standard MI. Moreover, when some language feature is considered as too expensive, the language should provide ways to restrict specialization: some classes might be specialized only in single or arborescent inheritance, some attribute or parameter types might not be overridden, and so on. Furthermore, in object orientation, modularity has been historically confused with the notion of class: however, the class is a conceptual unit, not always the program unit of the size appropriate for compilation. A higher level notion such as modules has been advocated for long [Szypersky 1992; Bracha and Lindstrom 1992]. JAVA packages are not a good answer as they are mainly designed as name spaces: however, current discussions on the notion of sealing may be a forward step towards true modules [Biberstein et al. 2001]. In any case, modular compilation of object-oriented languages will be a topic of further research. Hence, perspective is threefold: application of global techniques in separate compilation, modular compilation and global techniques compatible with incremental loading.

\section{REFERENCES}

Agesen, O., Freund, S., And Mitchell, J. 1997. Adding type parameterization to Java. See OOPSLA [1997], 
49-65.

Alpern, B., Attanasio, C., Barton, J., Cocchi, A., Hummel, S., Lieber, D., Ngo, T., Mergen, M., Shepherd, J., AND Smith, S. 1999. Implementing Jalapeño in Java. See OOPSLA [1999], 314-324.

Alpern, B., Cocchi, A., Fink, S., AND Grove, D. 2001. Efficient implementation of Java interfaces: Invokeinterface considered harmless. See OOPSLA [2001].

Alpern, B., Cocchi, A., And Grove, D. 2001. Dynamic type checking in Jalapeño. In USENIX Java Virtual Machine Research and Technology Symposium (JVM'01).

AmericA, P., Ed. 1991. Proceedings of the Fifth European Conference on Object-Oriented Programming, ECOOP'91. LNCS 512. Springer-Verlag.

Ancona, D., Lagorio, G., And ZuccA, E. 2000. JAM — a smooth extension of Java with mixins. See Bertino [2000], $154-178$.

André, P. AND Royer, J.-C. 1992. Optimizing method search with lookup caches and incremental coloring. In Proc. OOPSLA'92. SIGPLAN Notices, 27(10). ACM Press, Vancouver, 110-126.

Atkinson, R., Liskov, B., And Scheifler, R. 1978. Aspects of implementing CLU. In Proc. ACM 1978 Annual Conference. ACM Press.

Bacon, D., Fink, S., And Grove, D. 2002. Space- and time-efficient implementation of the java object model. In Proc. ECOOP'2002, J. M. Troya, Ed. LNCS. Springer-Verlag.

Bacon, D. And Sweeney, P. 1996. Fast static analysis of C++ virtual function calls. In Proc. OOPSLA'96. SIGPLAN Notices, 31(10). ACM Press, 324-341.

Bertino, E., Ed. 2000. Proceedings of the 14 th European Conference on Object-Oriented Programming, ECOOP'2000. LNCS 1850. Springer-Verlag.

Biberstein, M., Gil, J., And Porat, S. 2001. Sealing, encapsulation and mutability. See Knudsen [2001], 28-52.

Boeнм, H.-J. 1993. Space-efficient conservative garbage collection. In Proc. ACM Conf. on Programming Language Design and Implementation (PLDI'93). ACM SIGPLAN Notices, 28(6). 197-206.

Bracha, G. AND Cook, W. 1990. Mixin-based inheritance. In Proc. OOPSLA/ECOOP'90. SIGPLAN Notices, 25(10). ACM Press, 303-311.

Bracha, G. And Lindstrom, G. 1992. Modularity meets inheritance. In Proc. Int. Conf. on Computer Languages. IEEE, 282-290.

Bracha, G., Odersky, M., Stoutamire, D., And Wadler, P. 1998. Making the future safe for the past: Adding genericity to the Java programming language. See OOPSLA [1998], 183-200.

Bruce, K. B., Petersen, L., And Fiech, A. 1997. Subtyping is not a good "match" for object-oriented languages. In Proc. ECOOP'97, M. Aksit and S. Matsuoka, Eds. LNCS 1241. Springer-Verlag, 104-127.

Calder, B. And Grunwald, D. 1994. Reducing indirect function call overhead in c++ programs. In Proc. of ACM Symp. on Principles of Prog. Lang. (POPL'94). 397-408.

Cargill, T. A. 1991. Controversy: The case against multiple inheritance in C++. Computing Systems 4, 1, 69-82.

Cartwright, R. And Steele, G. 1998. Compatible genericity with run-time types for the Java programming language. See OOPSLA [1998], 201-215.

Castagna, G. 1995. Covariance and contravariance: Conflict without a cause. ACM Transactions on Programming Languages and Systems 17, 3, 431-437.

Castagna, G. 1997. Object-Oriented Programming: A Unified Foundation. Progress in Theorical Computer Science. Birkhäuser, Berlin.

Chambers, C. And Ungar, D. 1989. Customization: Optimizing compiler technology for SELF, a dynamicallytyped object-oriented language. See OOPSLA [1989], 146-160.

Cohen, N. 1991. Type-extension type tests can be performed in constant time. Programming languages and systems 13, 4, 626-629.

Collin, S., Colnet, D., And Zendra, O. 1997. Type inference for late binding. the SmallEiffel compiler. In Joint Modular Languages Conference. LNCS 1204. Springer Verlag, 67-81.

Colnet, D., Coucaud, P., And Zendra, O. 1998. Compiler support to customize the mark and sweep algorithm. In ACM Sigplan International Symposium on Memory Management (ISMM'98). 154-165.

Cook, W. R. 1989. A proposal for making Eiffel type-safe. In Proc. ECOOP'89, S. Cook, Ed. Cambridge University Press, 57-70.

Day, M., Gruber, R., Liskov, B., And Myers, A. 1995. Subtypes vs. where clauses. constraining parametric polymorphism. See OOPSLA [1995], 156-168.

Dixon, R., McKee, T., Schweitzer, P., And Vaughan, M. 1989. A fast method dispatcher for compiled languages with multiple inheritance. See OOPSLA [1989].

Driesen, K. 1999. Software and hardware techniques for efficient polymorphic calls. Ph.D. thesis, University of California, Santa Barbara.

Driesen, K. ANd Hölzle, U. 1995. Minimizing row displacement dispatch tables. See OOPSLA [1995], 141-155.

Driesen, K., Hölzle, U., And Vitek, J. 1995. Message dispatch on pipelined processors. In Proc. ECOOP'95, W. Olthoff, Ed. LNCS 952. Springer-Verlag, 253-282. 
Ducournau, R. 1991. Yet Another Frame-based Object-Oriented Language: YAFOOL Reference Manual. Sema Group, Montrouge, France.

Ducournau, R. 1997. La compilation de l'envoi de message dans les langages dynamiques. L'Objet 3, 3, $241-276$. Ducournau, R. 2002a. La coloration pour l'implémentation des langages à objets à typage statique. In Actes LMO'2002 in L'Objet vol. 8, M. Dao and M. Huchard, Eds. Hermès, 79-98.

Ducournau, R. 2002b. "Real World" as an argument for covariant specialization in programming and modeling. In Advances in Object-Oriented Information Systems, OOIS'02 Workshops Proc., J.-M. Bruel and Z. Bellahsène, Eds. LNCS 2426. Springer-Verlag, 3-12.

Ducournau, R., Habib, M., Huchard, M., And Mugnier, M.-L. 1994. Proposal for a monotonic multiple inheritance linearization. In Proc. OOPSLA'94. ACM Press.

ECKel, N. AND GIL, J. 2000. Empirical study of object-layout and optimization techniques. See Bertino [2000], $394-421$.

Ellis, M. And Stroustrup, B. 1990. The Annotated $C++$ Reference Manual. Addison-Wesley, Reading (MA), USA.

Ernst, E. 2002. Call by declaration. In Proc. ECOOP'2002 Inheritance Workshop, A. P. Black, E. Ernst, P. Grogono, and M. Sakkinen, Eds. University of Jyväskylä, 44-50.

Gagnon, E. M. And Hendren, L. 2001. SableVM: A research framework for the efficient execution of Java bytecode. In USENIX Java Virtual Machine Research and Technology Symposium (JVM'01).

Garey, M. And Johnson, D. 1979. Computers and Intractability. A Guide to the Theory of NP-Completeness. W.H. Freeman and Company, San Francisco (CA), USA.

GiL, J. And Itai, A. 1998. The complexity of type analysis of object oriented programs. In Proc. ECOOP'g8. LNCS 1445. Springer-Verlag, 601-634.

Gil, J. AND SweEney, P. 1999. Space and time-efficient memory layout for multiple inheritance. See OOPSLA [1999], 256-275.

Goldberg, A. And Robson, D. 1983. Smalltalk-80, the Language and its Implementation. Addison-Wesley, Reading (MA), USA.

Gosling, J., Joy, B., And Steele, G. 1996. The JAVA Language Specification. Addison-Wesley.

Grand, M. 1997. JAVA Language Reference. O'Reilly.

Grove, D. And Chambers, C. 2001. A framework for call graph construction algorithms. ACM Trans. Program. Lang. Syst. 23, 6, 685-746.

Hölzle, U., Chambers, C., And Ungar, D. 1991. Optimizing dynamically-typed object-oriented languages with polymorphic inline caches. See America [1991], 21-38.

Huchard, M. and Leblanc, H. 2000. Computing interfaces in Java. In Proc. of IEEE Int. Conf. on Automated Software Engineering (ASE'2000). 317-320.

Jones, R. And Lins, R. 1996. Garbage Collection. Wiley.

Knudsen, J. L., Ed. 2001. Proceedings of the 15th European Conference on Object-Oriented Programming, ECOOP'2001. LNCS 2072. Springer-Verlag.

Koenig, A. 1998. Standard - the C++ language. Report ISO/IEC 14882:1998, Information Technology Council (NCTIS). http://www.nctis.org/cplusplus.htm.

Krall, A. And Grafl, R. 1997. CACAO - a 64 bits JavaVM just-in-time compiler. Concurrency: Practice ans Experience 9, 11, 1017-1030.

Kristensen, B., Lehrmann Madsen, O., Møller-Pedersen, B., And NygaArd, K. 1987. The BetA programming language. In Research Directions in Object Oriented Programming, B. Shriver and P. Wegner, Eds. MIT Press, Cambridge (MA), USA, 7-48.

Krogdahl, S. 1985. Multiple inheritance in Simula-like languages. BIT 35, 318-326.

Lalande, A. 1926. Vocabulaire technique et critique de la philosophie. Presses Universitaires de France, Paris.

Lippman, S. 1996. Inside the $C++$ Object Model. Addison-Wesley, New York.

Liskov, B., Curtis, D., Day, M., Ghemawat, S., Gruber, R., Johnson, P., And Myers, A. C. 1995. ThetA reference manual. Technical report, MIT.

Lorenz, M. And KIDD, J. 1994. Object-Oriented Software Metrics. Prentice-Hall, Englewood Cliffs (NJ), USA.

Meyer, B. 1992. Eiffel: The Language. Prentice Hall Object-Oriented Series. Prentice Hall International, Hemel Hempstead, UK.

Meyer, B. 1997. Object-Oriented Software Construction, second ed. The Object-Oriented Series. Prentice-Hall, Englewood Cliffs (NJ), USA.

Meyer, B. 2001. Overloading vs. object technology. Journal of Object-Oriented Programming 14, 5 (October/November), 3-7.

Meyer, J. And Downing, T. 1997. JAVA Virtual Machine. O'Reilly.

Microsoft. 2001. C\# Language specifications, v0.28. Tech. rep., Microsoft Corporation.

Muthukrishnan, S. And Muller, M. 1996. Time and space efficient method lookup for object-oriented languages. In Proc. ACM-SIAM Symp. on Discrete Algorithms. ACM/SIAM, 42-51.

Myers, A. 1995. Bidirectional object layout for separate compilation. See OOPSLA [1995], 124-139.

RR LIRMM 2002-174 
Myers, A. AND Liskov, B. 1994. Efficient implementation of parameterized types despite subtyping. Thor note 9, LCS, MIT.

Odersky, M. And Wadler, P. 1997. Pizza into Java: Translating theory into practice. In Proceedings of the 24th ACM Symposium on Principles of Programming Languages (POPL'97), Paris, France. ACM Press, New York (NY), USA, 146-159.

OOPSLA 1989. Proceedings of the Fourth ACM Conference on Object-Oriented Programming, Languages and Applications, OOPSLA'89. ACM Press, New Orleans.

OOPSLA 1995. Proceedings of the Tenth ACM Conference on Object-Oriented Programming, Languages and Applications, OOPSLA'95. SIGPLAN Notices, 30(10). ACM Press.

OOPSLA 1997. Proceedings of the Twelfth ACM Conference on Object-Oriented Programming, Languages and Applications, OOPSLA'97. SIGPLAN Notices, 32(10). ACM Press.

OOPSLA 1998. Proceedings of the Thirteenth ACM Conference on Object-Oriented Programming, Languages and Applications, OOPSLA'98. SIGPLAN Notices, 33(10). ACM Press.

OOPSLA 1999. Proceedings of the Fourteenth ACM Conference on Object-Oriented Programming, Languages and Applications, OOPSLA'99. SIGPLAN Notices, 34(10). ACM Press.

OOPSLA 2001. Proceedings of the Sixteenth ACM Conference on Object-Oriented Programming, Languages and Applications, OOPSLA'01. SIGPLAN Notices, 36(10). ACM Press.

Privat, J. 2002. Analyse de types et graphe d'appels en compilation séparée. M.S. thesis, Université Montpellier II.

Pugh, W. And Weddel, G. 1990. Two-directional record layout for multiple inheritance. In Proc. ACM Conf. on Programming Language Design and Implementation (PLDI'90). ACM SIGPLAN Notices, 25(6). 85-91.

QueInneC, C. 1997. Fast and compact dispatching for dynamic object-oriented languages. Information Processing Letters.

Raynaud, O. And Thierry, E. 2001. A quasi optimal bit-vector encoding of tree hierarchies. application to efficient type inclusion tests. See Knudsen [2001], 165-180.

Rossie, J. G., Friedman, D. P., And Wand, M. 1996. Modeling subobject-based inheritance. In Proc. ECOOP'96, P. Cointe, Ed. LNCS 1098. Springer-Verlag.

SAKkinen, M. 1992. A critique of the inheritance principles of C++. Computing Systems 5, 1, 69-110.

Schubert, L., Papalaskaris, M., And Taugher, J. 1983. Determining type, part, color and time relationships. Computer 16, 10, 53-60.

Simon, R., Stapf, E., Mingins, C., And Meyer, B. 2000. Eiffel for e-commerce under .NET. Journal of ObjectOriented Programming 13, 5 (October), 42-47.

SNyder, A. 1991. Modeling the C++ object model: An application of an abstract object model. See America [1991], $1-20$.

Solorzano, J. H. And Alagić, S. 1998. Parametric polymorphism for Java: A reflective solution. See OOPSLA [1998], 216-225.

Steele, G. 1990. Common Lisp: The Language, Second Edition. Digital Press, Bedford (MA), USA.

Stefik, M. And Bobrow, D. 1986. Object-oriented programming: Themes and variations. The AI Magazine 6, 4, 40-62.

Sweeney, P. And Burke, M. 1998. A methodology for quantifying and evaluating the space overhead in C ++ object models. Tech. Rep. RC21370, IBM T.J. Watson Research Center.

SzYPERSKY, C. 1992. Import is not inheritance. Why we need both: Modules and classes. In Proc. ECOOP'92, O. L. Madsen, Ed. LNCS 615. Springer-Verlag, 19-32.

Szypersky, C., Oмоhundro, S., And Murer, S. 1994. Engineering a programming language: The type and class system of Sather. In Proc. of First Int. Conference on Programming Languages and System Architectures. LNCS 782. Springer Verlag.

Vitek, J. And Horspool, R. 1994. Taming message passing: efficient method look-up for dynamically typed languages. In Proc. ECOOP'94, M. Tokoro and R. Pareschi, Eds. LNCS 821. 432-449.

Vitek, J., Horspool, R., And Krall, A. 1997. Efficient type inclusion tests. See OOPSLA [1997], $142-157$.

Waldo, J. 1991. Controversy: The case for multiple inheritance in C++. Computing Systems 4, 2, $157-171$.

Wang, T. And Smith, S. 2001. Precise constraint-based type inference for java. See Knudsen [2001], 99-117.

WeBer, F. 1992. Getting class correctness and system correctness equivalent - how to get covariant right. In Technology of Object-Oriented Languages and Systems (TOOLS 8), R. Ege, M. Singh, and B. Meyer, Eds. $192-213$.

Wilson, P. R. 1992. Uniprocessor garbage collection techniques. In Int. Workshop on Memory Management (IWMM'92). LNCS 637. Springer Verlag.

Zendra, O., Colnet, D., And Collin, S. 1997. Efficient dynamic dispatch without virtual function tables: The SmallEiffel compiler. In Proceedings of OOPSLA'9\%, Atlanta (GA), USA. special issue of ACM SIGPLAN Notices, 32(10). 125-141.

Zibin, Y. AND GiL, J. 2001. Efficient subtyping tests with PQ-encoding. See OOPSLA [2001], 96-107. 
Zibin, Y. AND GIL, J. 2002. Fast algorithm for creating space efficient dispatching tables with application to multi-dispatching. In Proc. OOPSLA'02. SIGPLAN Notices, 37(10). ACM Press. 


\section{APPENDIX}

\section{A. SPACE BENCHMARKS}

Some large benchmarks are commonly used in the object-oriented implementation community ${ }^{16}$, e.g. by [Driesen and Hölzle 1995; Vitek et al. 1997; Eckel and Gil 2000; Zibin and Gil 2002]. We present here some statistics computed from these benchmarks according to various implementation techniques.

Four techniques have been considered: ideal SST implementation, even if non applicable in all benchmarks, pure NVI, the ideal devirtualization scheme of section 7.3.2 (DVI), MI with the empty subobject optimization (ESO) and standard MI (SMI). DVI may be understood as the best $\mathrm{C}++$-like implementation ${ }^{17}$, with a sound multiple inheritance semantics, for a hand made program, with a complete knowledge of the whole program. On the contrary, ESO is presumably the best standard MI implementation in separate compilation. No statistics on MST have been made as available JAVA benchmarks don't distinguish classes and interfaces.

\section{A.1 Benchmark description, interpretation and correction}

Each benchmark is a file of class descriptions, each of which consists of four items: the class name, the list of its direct superclasses, the two lists of attributes and methods defined in the class. They have been produced and used mostly for assessing techniques for subtyping test and method call, so they often don't comport informations about attributes. Therefore, we restrict hereafter our statistics to the few of them which comport such data. Digitalk is a SMaldtalk distribution ${ }^{18}$, whereas Unidraw is a $\mathrm{C}++$ program mainly in SI, Lov and Geode are EIFFEL-like programs making an intensive use of MI.

The contents of the benchmarks is also questionable. One should expect that only pure objectoriented data are included: typically, static methods and variables, or non virtual methods should be excluded. One will see that this is not the case with the JDK benchmark which includes obviously static variables and we can only certify that non object-oriented data has been removed from the SMALL EIFFEL benchmarks. Besides that, name interpretation may be discussed as it is different between the different languages: in $\mathrm{C}++$ and JAVA benchmarks, parameter types have been concatenated to method names in order to deal with static overloading. However, the case of attribute is not clear: the same attribute name in two related classes has been interpreted as overriding (as in EIFFEL), not as overloading (as in C ++ or JAVA): hence, Unidraw and JDK may be slightly underestimated. Renaming, which is proper to EIFFEL, has not been considered in the SMALl EIfFel benchmark ${ }^{19}$, and no information is available about Lov or Geode.

Moreover, these benchmarks are often libraries, not single applications: it is thus difficult to extrapolate from them the size appropriate for typical applications and to judge the maximal number of classes, methods, attributes, etc. of applications. Anyway, some hundreds of classes seem common in object-oriented programs, e.g. the SMALL EIFFEL compiler [Zendra et al. 1997].

Finally, the statistics may differ from previous ones in several ways. Some are connected to the fact that this paper aims at separate compilation and static typing. First, there is no definition overloading (see note 4, page 5): two methods or attributes with the same name, introduced in two unrelated classes, are considered as different ${ }^{20}$. Second, all methods are considered, whereas Yoav Zibin's benchmarks remove degenerate methods, i.e. methods which have only one definition.

Furthermore, some measures are uniquely determinate (SMI) whereas many other (ESO, DVI, coloring and even NVI) are the result of an optimization: heuristics commonly used are greedy and there is a very little chance that two different experiments give the same number. NVI, DVI

\footnotetext{
${ }^{16}$ Many people contributed to those benchmarks, among which Karel Driesen and Jan Vitek: a current repository is Yoav Zibin's web site, http://www.cs.technion.ac.il/ zyoav/.

${ }_{17}$ However, as noticed in section 4.1.3, actual $\mathrm{C}++$ implementation would be less efficient (see Table XIV).

18 As Smalltalk is dynamically typed, these statistics are not directly applicable.

19 In Eiffel, it may be difficult to distinguish attributes and methods: constant features, i.e. features whose body is a constant, have been treated as methods, except when they are frozen.

${ }^{20}$ In JAVA, the same method may be introduced in two unrelated interfaces (definition overloading) but this has no effect on statistics.
} 
Table V. Statistics on classes, including empty and virtual classes, and inheritance edges, including virtual edges according to DVI scheme

\begin{tabular}{l|rrrrrr|rr}
\hline & \multicolumn{6}{|c|}{ classes } & \multicolumn{3}{c}{ edges } \\
name & total & \multicolumn{1}{|c}{ empty } & virtual & e+v & leaves & total & virtual \\
\hline SmallEiffel & 382 & 27 & 91 & 4 & 9 & 299 & 416 & 13 \\
JDK.1.0.2 & 604 & 96 & 153 & 5 & 11 & 445 & 650 & 20 \\
digitalk3 & 1357 & 430 & 539 & 0 & 0 & 923 & 1356 & 0 \\
digitalk2 & 535 & 156 & 235 & 0 & 0 & 381 & 534 & 0 \\
Unidraw & 614 & 115 & 81 & 3 & 1 & 481 & 623 & 4 \\
Lov-obj-ed & 436 & 39 & 132 & 21 & 46 & 218 & 747 & 68 \\
Geode & 1318 & 163 & 436 & 78 & 115 & 732 & 2486 & 251 \\
\hline Total & 5253 & 1026 & 1673 & 113 & 184 & 3480 & 6820 & 358 \\
\hline
\end{tabular}

Table VI. Statistics (total number, mean and max) on attribute number, either introduced or inherited

\begin{tabular}{l|rrr|rrr} 
name & \multicolumn{3}{|c|}{ introduced } & \multicolumn{3}{|c}{ inherited } \\
\hline SmallEiffel & 1317 & 3.4 & 39 & 3441 & 9.0 & 46 \\
JDK.1.0.2 & 2192 & 3.6 & 274 & 90667 & 150.1 & 600 \\
digitalk3 & 1495 & 1.1 & 37 & 11352 & 8.4 & 52 \\
digitalk2 & 529 & 1.0 & 30 & 3885 & 7.3 & 30 \\
Unidraw & 1574 & 2.6 & 36 & 5101 & 8.3 & 47 \\
Lov-obj-ed & 1262 & 2.9 & 74 & 3552 & 8.1 & 105 \\
Geode & 2919 & 2.2 & 182 & 14355 & 10.9 & 217 \\
\hline Total & 11288 & 2.1 & 274 & 132353 & 25.2 & 600
\end{tabular}

Table VII. Statistics (total, mean and maximum) on subobject numbers

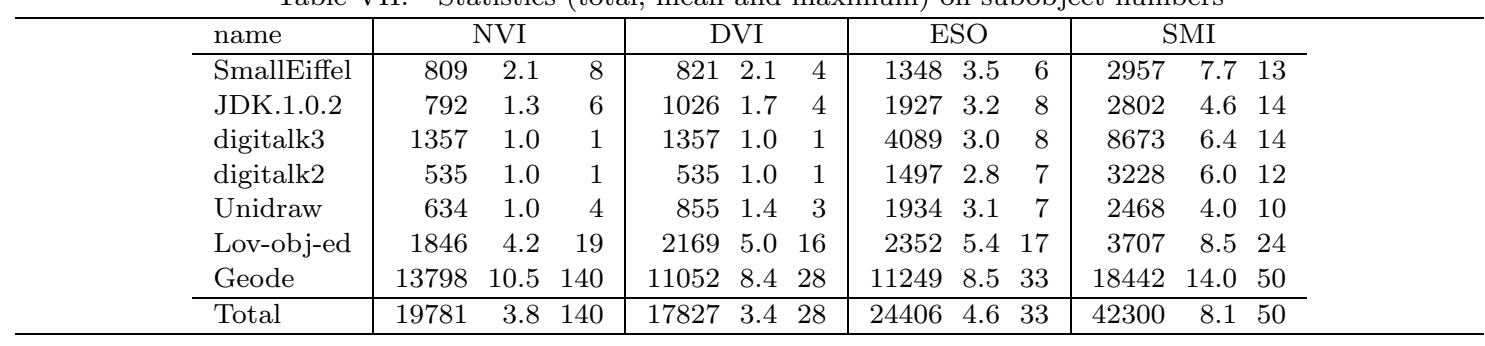

and ESO depend on the choice of the direct superclass whose layout is expanded by the current class: in all three cases, our heuristics choose the class with the greatest number of methods, which tends to minimize table size. NVI and ESO are then almost uniquely determinate. As for devirtualization, no optimization is made.

However, besides all those small flaws, the statistics presented hereafter are a somewhat precise indication of the relative cost of the measured techniques.

\section{A.2 Dynamic space}

Table V presents the number of classes, together with the number of classes without attributes, in the two kinds of merging (see section 3.3), and the number of virtual classes according to the DVI scheme (see section 7.3.2). The number of classes without attributes is surprisingly high in all benchmarks, between 30 and $60 \%$. It was unexpected and it will have an important effect on the number of subobjects as empty subobjects may be merged into another one. On the contrary, there are very few virtual classes and virtual edges. Furthermore, the number of classes with no attribute or non virtual direct superclass (column "e+v") is quite small, as would be the benefit of combining ESO and DVI.

Table VI presents statistics on the number of attributes, as well introduced in a class as the total number for the class. JAVA figures obviously deviate from other statistics: the reason is that static variables are taken into account, as well as attributes.

Attribute number is to compare with the number of subobjects, i.e. pointers to method tables in the object layout: this gives a good idea of the dynamic overhead of MI implementations (Table VII). On the whole, pointers to method tables are more than doubling dynamic space as soon as inheritance is intensively used (Lov and Geode) but ESO and DVI reduces the overhead by 
Table VIII. Statistics on upcast table size and VBPTRs (total, mean and max)

\begin{tabular}{|c|c|c|c|c|c|c|c|c|}
\hline \multirow{2}{*}{$\begin{array}{l}\text { name } \\
\text { SmallEiffel }\end{array}$} & \multicolumn{2}{|c|}{ e-VBPTRs } & \multicolumn{2}{|c|}{ i-VBPTRs } & \multicolumn{2}{|c|}{ DVI } & ESO & SMI \\
\hline & 438 & 1. 3 & 1647 & 4. 18 & 1647 & 4. 18 & $\begin{array}{lll}4595 & 12 . & 44\end{array}$ & 1053228.76 \\
\hline JDK.1.0.2 & 448 & 1. & 478 & 1. 9 & 478 & 1. 9 & $4992 \quad 8 . \quad 49$ & 6465 11. 61 \\
\hline digitalk3 & 0 & 0. & 0 & 0. & 0 & 0. & $2031515 . \quad 55$ & 2663320.91 \\
\hline digitalk2 & 0 & 0. & 0 & 0. & 0 & 0. & $7160 \quad 13 . \quad 45$ & 9198 17. 66 \\
\hline Unidraw & 244 & 0.3 & 264 & 0. 7 & 264 & 0. 7 & $3310 \quad 5 . \quad 28$ & $\begin{array}{lll}4711 & 8 . & 45\end{array}$ \\
\hline Lov-obj-ed & 2326 & 5. 27 & 2808 & 6. 39 & 2951 & 7. 40 & 1011123.117 & 11503 26. 118 \\
\hline Geode & 22854 & 17. 135 & 29191 & 22. 189 & 31616 & 24. 190 & $89547 \quad 68.481$ & $94992 \quad 72.490$ \\
\hline
\end{tabular}

Table IX. Statistics on method number

\begin{tabular}{l|rrr|rrr|rrr}
\hline & \multicolumn{3}{|c|}{ introduced } & \multicolumn{3}{c|}{ defined } & \multicolumn{3}{c}{ inherited } \\
name & total & mean & $\max$ & total & mean & $\max$ & total & mean & max \\
\cline { 2 - 2 } & 3832 & 10.0 & 209 & 6482 & 17.0 & 209 & 37658 & 98.6 & 277 \\
JDK.1.0.2 & 3190 & 5.3 & 75 & 5095 & 8.4 & 78 & 22348 & 37.0 & 156 \\
digitalk3 & 13007 & 9.6 & 440 & 17104 & 12.6 & 460 & 613675 & 452.2 & 1065 \\
digitalk2 & 5536 & 10.3 & 271 & 6858 & 12.8 & 272 & 154616 & 289.0 & 677 \\
Unidraw & 1751 & 2.9 & 103 & 3327 & 5.4 & 103 & 14167 & 23.1 & 123 \\
Lov-obj-ed & 3631 & 8.3 & 117 & 5026 & 11.5 & 127 & 36883 & 84.6 & 287 \\
Geode & 8078 & 6.1 & 193 & 14214 & 10.8 & 207 & 302742 & 229.7 & 870 \\
\hline Total & 39035 & 7.4 & 440 & 58122 & 11.1 & 460 & 1182122 & 225.0 & 1065 \\
\hline
\end{tabular}

$40 \%$. Of course, DVI is better than any other technique, as it is near optimal. One should not be surprised by the fact that NVI is worse than ESO in the case of Geode: the worst-case complexity of NVI is exponential. Furthermore, empty subobjects bring no optimization to NVI.

Table VIII shows the size occupied, either by upcast tables in method tables, or by VBPTRs in object layout, the latter in the DVI case only (see section 4.3). Besides JAVA because of static variables, this demonstrates that VBPTRs have a very large overhead with SMI. ESO has a poor effect, with a $600 \%$ overhead: this is due to the fact that empty classes are mostly of the second kind, which does not save on upcast tables. On the contrary, DVI is a great improvement: upcast tables are 3 times smaller in the worst case, and VBPTRs are still fewer. On the whole, in the worst case (Geode), DVI with e-VBPTRs is more than doubling dynamic space w.r.t. SST, which is larger than the expected overhead of attribute coloring. On average, the difference between e-VBPTRs and i-VBPTRs is not significant.

If one accepts doubling (Geode), or almost doubling (SmallEiffel, Lov) dynamic memory, one should envisage inline table cache (see section 4.5), which is stricly less than doubling dynamic memory as monomorphic attributes don't need tables. Unfortunately, benchmarks include no information on polymorphic/monomorphic attributes. One must however notice that collections, i.e. arrays, are not taken in account in our statistics: the cost of doubling arrays instead of adding a small constant overhead may be larger than doubling standard objects.

On the whole, MI overhead in the object layout may be less than the one that previous works as [Sweeney and Burke 1998; Gil and Sweeney 1999; Eckel and Gil 2000] report, as they consider only the hierarchy structure whereas data on attributes and methods is needed to deal with empty subobjects. As a matter of fact, though most benchmarks are common, it is impossible to compare our statistics with those of Eckel and Gil [2000] because they don't include any per benchmark statistics of either the subobject number or the upcast tables size.

\section{A.3 Static space}

Table IX presents statistics on the number of methods, as well introduced or defined in a class as the total number for the class. 
Table X. Statistics on table size (in K-entries)

\begin{tabular}{l|rrrrr|rrrrr}
\multicolumn{10}{c}{ Table X. } & Statistics on table size (in K-entries) \\
\hline & SST & NVI & DVI & ESO & SMI & & & & \\
name & $(s)$ & $(n v)$ & $(d v)$ & $(m)$ & $\left(m^{\prime}\right)$ & $n v / s$ & $d v / s$ & $m / d v$ & $m^{\prime} / m$ & $m / s$ \\
\hline SmallEiffel & 38 & 59 & 61 & 100 & 188 & 1.6 & 1.6 & 1.6 & 1.9 & 2.7 \\
JDK.1.0.2 & 22 & 25 & 29 & 55 & 77 & 1.1 & 1.3 & 1.9 & 1.4 & 2.4 \\
digitalk3 & 614 & 614 & 614 & 1739 & 3154 & 1.0 & 1.0 & 2.8 & 1.8 & 2.8 \\
digitalk2 & 155 & 155 & 155 & 391 & 729 & 1.0 & 1.0 & 2.5 & 1.9 & 2.5 \\
Unidraw & 14 & 14 & 14 & 31 & 40 & 1.0 & 1.0 & 2.2 & 1.3 & 2.2 \\
Lov-obj-ed & 37 & 81 & 92 & 122 & 183 & 2.2 & 2.5 & 1.3 & 1.5 & 3.3 \\
Geode & 303 & 1093 & 925 & 1235 & 1894 & 3.6 & 3.1 & 1.3 & 1.5 & 4.1 \\
\hline Total & 1182 & 2042 & 1889 & 3671 & 6264 & 1.7 & 1.6 & 1.9 & 1.7 & 3.1 \\
\hline
\end{tabular}

Method tables. According to the different techniques, the total size of method tables may be computed by the following formulas, where $m_{C}$ is the number of methods introduced by $C$ :

$$
\sum_{C} f_{C}, \text { where } f_{C}= \begin{cases}M_{C}=\sum_{C \preceq D} m_{D} & \text { SST } \\ m_{C}^{\prime}+\sum_{C \prec_{\mathrm{d}} D} f_{D} & \text { NVI } \\ \sum_{C \preceq D} M_{D} & \text { SMI } \\ \sum_{C \preceq_{e s o} D} M_{D} & \text { ESO }\end{cases}
$$

In the NVI case, $m_{C}^{\prime}=M_{C}-M_{D}$ where $C \prec_{\mathrm{e}} D$. The SMI case can be rewritten as $\sum_{D} n_{D} M_{D}$, where $n_{D}$ is the number of $D$ subclasses, including $D$. The ESO case amounts to exclude merged classes from the sum on $D: C \preceq$ eso $D$ means that $C \preceq D$ and there is no $D^{\prime}$ merged to $D$, i.e. empty and such that $C \preceq D^{\prime} \prec_{\mathrm{d}} D$. In the DVI case, the formula is more complicated:

$$
f_{C}=f_{C}^{\prime}+\sum_{C \unlhd_{v} D} f_{D}^{\prime} \text { where } f_{C}^{\prime}=m_{C}^{\prime}+\sum_{C \prec_{\mathrm{nv}} D} f_{D}^{\prime}
$$

where $C \preceq_{v} D$ iff $C \preceq D$ and $\nexists D^{\prime}, C \preceq D^{\prime} \prec_{\text {nv }} D$ (see section 4.1 .3 for the definition of relations $\prec_{\mathrm{v}}, \prec_{\mathrm{nv}}$ and $\left.\prec_{\mathrm{e}}\right)$.

Table X shows the total size, i.e. number of entries, of method tables in all techniques. The ratio between ESO and ideal SST table sizes is around 3-4. When MI is intensively used, the difference between ESO and DVI is significant but less than expected: in fact, ESO improves upon SMI with the same ratio as DVI upon ESO. The advantage of DVI w.r.t. SMI or ESO lies in the null thunks which may be measured by the ratio $(d v-s) /(m-s)$ : this ratio is around $40 \%$ in case of intensive use of MI (Geode and Lov). As for pure NVI, besides its unsound semantics, its bad worst-case complexity tends to reduce the gap with SMI and ESO. NVI may be worse than ESO or SMI: in Geode, the NVI number of subobjects is far greater than with SMI in the maximal case, and than with ESO in average (Table VII).

For an exact assessment of MI overhead, one must also take into account shifts to receivers, which can be handled by thunks or by 2 extra instructions in the code plus a $50 \%$ increasing of table size (if one uses only short integers). The thunk number is exactly the difference of table sizes between the considered MI technique and SST: there is a thunk per table entry, but for each class-method pair there is exactly one null shift. One must also add, from Table VIII, the upcast tables size $(u)$ to SMI, ESO and DVI sizes.

A conservative estimate of method coloring consists in adding to SST table size the superclass number ( $s p$, i.e. the subobject number for SST, Table VII) for simulating accessors, and in majoring the total by $50 \%$, which is an upper bound for all experiments. In case of SI hierarchies (Digitalk), the $50 \%$ majoration is not needed as coloring is exactly SST.

Code size. Statistics on the number of method call sites in the method code are missing, as well as for any other mechanism: hence, there is no way to measure the static space occupied by the different techniques in the method code. However, previous studies show that the number of call sites may be large enough to make code size significant w.r.t. table size [Driesen et al. 1995; Ducournau 1997]. For instance, a number of 35042 call sites is reported for a SMALLTALK implementation with 774 classes and 8540 method definitions. Assuming an average number of 4 call sites per method definition - which may be quite unlikely in $\mathrm{C}++$ or EIfFEL programs - Table XI RR LIRMM 2002-174 
Table XI. Estimate of code size for method calls (in K-entries)

\begin{tabular}{l|rrr|rr|rr}
\hline name & classes & methods & calls $(c)$ & code $(3 c)$ & tables $(m)$ & shifts & thunks \\
\cline { 2 - 2 } & 382 & 6482 & 25928 & 78 & 100 & 102 & 124 \\
JDK.1.0.2 & 604 & 5095 & 20380 & 61 & 55 & 68 & 64 \\
digitalk3 & 1357 & 17104 & 68416 & 205 & 1739 & 1006 & 2251 \\
digitalk2 & 535 & 6858 & 27432 & 82 & 391 & 250 & 472 \\
Unidraw & 614 & 3327 & 13308 & 40 & 31 & 42 & 33 \\
Lov-obj-ed & 436 & 5026 & 20104 & 60 & 122 & 101 & 170 \\
Geode & 1318 & 14214 & 56856 & 171 & 1235 & 731 & 1864 \\
\hline Total & 5253 & 58122 & 232488 & 697 & 3671 & 2301 & 4979 \\
\hline
\end{tabular}

Table XII. Final comparison between SST, coloring, NVI and DVI (both with thunks), and between shifts and thunks in the ESO case (in K-entries)

\begin{tabular}{l|rrrrrr|rrrrr}
\hline & \multicolumn{4}{|c|}{ total size } & \multicolumn{4}{c}{ ratio vs. SST } \\
name & SST & color & NVI & DVI & shifts & thunks & color & NVI & DVI & shifts & thunks \\
\hline SmallEiffel & 115 & 136 & 180 & 186 & 282 & 304 & 1.2 & 1.6 & 1.6 & 2.4 & 2.6 \\
JDK.1.0.2 & 83 & 97 & 93 & 102 & 186 & 182 & 1.2 & 1.1 & 1.2 & 2.2 & 2.2 \\
digitalk3 & 819 & 823 & 819 & 819 & 2961 & 4206 & 1.0 & 1.0 & 1.0 & 3.6 & 5.1 \\
digitalk2 & 237 & 239 & 237 & 237 & 727 & 949 & 1.0 & 1.0 & 1.0 & 3.1 & 4.0 \\
Unidraw & 54 & 63 & 54 & 54 & 114 & 105 & 1.2 & 1.0 & 1.0 & 2.1 & 1.9 \\
Lov-obj-ed & 97 & 118 & 230 & 264 & 288 & 357 & 1.2 & 2.4 & 2.7 & 3.0 & 3.7 \\
Geode & 473 & 639 & 2845 & 2356 & 2181 & 3313 & 1.3 & 6.0 & 5.0 & 4.6 & 7.0 \\
\hline
\end{tabular}

shows that the 3 instruction sequence code for method call in standard SST has a rather significant global impact on static space. For assessing the static space overhead or gain of thunks, one must compare the cost of the shifts in the code and in the table, $x=2 c+m / 2$, with the cost of thunks, $x=2(m-s)$. The total static size will be obtained with $3 c+m+x+u / 2$, which must be compared to $3 c+3 s / 2+s p / 2$ which is an upper bound for coloring, and to $3 c+3 d v-2 s$ for DVI $(\text { Table XII })^{21}$. Thus, even when taking into account the size of the code, which is advantageous for thunks, the thunk space overhead remains greater.

One must notice, from Tables VI and IX, that the number of attributes and methods in a class is far from $2^{15}$ : thus, an implementation of $\Delta$ s and offsets with short integers is valid. This explains why $s p$ and $u$ are divided by 2 in the previous formula. However, treating attribute and method offsets, or class identifiers, as immediate values may depend on the processor. According to Driesen et al. [1995], current processors may offer from 8 to 13 bits for immediate values: 13 bits are sufficient for treating all that data as immediate values, but 8 bits are not enough for large applications. This is not a problem when offsets are computed at compile-time: the code will then differ according to the offset value, with a small overhead when the value is greater than 128 . However, when offsets are computed at link-time, the compiler should be able to predict whether the link-time computation will always produce a 8-bit value: thus, the overhead will be larger.

Abstract classes. Many other data are missing, for instance the number of abstract classes, without proper instances, which would reduce the static space estimate, as in the $\Sigma_{C}$ formula (13), $C$ should exclude abstract classes. When the class number is large, it is likely that many classes are abstract: the effect of a precise measure might be as significant as for classes without attributes. A common assumption, which may be far from reality, is that only leaves may have instances: according to Table V, one third of all classes would be abstract. This is an upper bound, twice the rate advocated by [Lorenz and Kidd 1994]. Table XIII presents the same statistics as Table X, according to this assumption. The effect of abstract classes is important, but the variation of the ratios between the different implementations is not significant.

Of course, abstract classes must be syntactically distinguished if one wants to gain from them, either by being declared abstract, as in SMALLTALK, or through the existence of some deferred feature, as in EIFFEL. 
Table XIII. Statistics on table size, when all non leaf classes are abstract (in K-entries)

\begin{tabular}{l|rrrrr|rrrrr}
\hline & SST & NVI & DVI & ESO & SMI & & & & \\
name & $(s)$ & $(n v)$ & $(d v)$ & $(m)$ & $\left(m^{\prime}\right)$ & $n v / s$ & $d v / s$ & $m / d v$ & $m^{\prime} / m$ & $m / s$ \\
\hline SmallEiffel & 30 & 47 & 48 & 82 & 153 & 1.6 & 1.6 & 1.7 & 1.9 & 2.7 \\
JDK.1.0.2 & 17 & 19 & 21 & 43 & 60 & 1.2 & 1.3 & 2.0 & 1.4 & 2.6 \\
digitalk3 & 411 & 411 & 411 & 1167 & 2178 & 1.0 & 1.0 & 2.8 & 1.9 & 2.8 \\
digitalk2 & 109 & 109 & 109 & 276 & 524 & 1.0 & 1.0 & 2.5 & 1.9 & 2.5 \\
Unidraw & 11 & 11 & 11 & 23 & 31 & 1.0 & 1.0 & 2.2 & 1.3 & 2.2 \\
Lov-obj-ed & 21 & 48 & 53 & 75 & 111 & 2.3 & 2.6 & 1.4 & 1.5 & 3.6 \\
Geode & 175 & 635 & 495 & 693 & 1083 & 3.6 & 2.8 & 1.4 & 1.6 & 4.0 \\
\hline Total & 773 & 1281 & 1148 & 2358 & 4141 & 1.7 & 1.5 & 2.1 & 1.8 & 3.0 \\
\hline
\end{tabular}

Table XIV. Comparison between DVI (left) and $\mathrm{C}++$ (right) devirtualization schemes

\begin{tabular}{|c|c|c|c|c|c|c|c|c|c|c|}
\hline name & \multicolumn{2}{|c|}{ virtual edges } & \multicolumn{2}{|c|}{ subobjects } & \multicolumn{2}{|c|}{ e-VBPTRs } & \multicolumn{2}{|c|}{ cast table } & \multicolumn{2}{|c|}{ table size } \\
\hline SmallEiffel & 13 & 22 & 821 & 1215 & 438 & 1041 & 1647 & 3123 & 61 & 81 \\
\hline JDK.1.0.2 & 20 & 43 & 1026 & 1167 & 448 & 652 & 478 & 675 & 29 & 31 \\
\hline Unidraw & 4 & 9 & 855 & 874 & 244 & 266 & 264 & 296 & 14 & 14 \\
\hline Lov-obj-ed & 68 & 107 & 2169 & 2438 & 2326 & 3364 & 2951 & 4106 & 92 & 106 \\
\hline Geode & 251 & 417 & 11052 & 13113 & 22854 & 35506 & 31616 & 44733 & 925 & 1091 \\
\hline Total & 358 & 602 & 17827 & 20719 & 26315 & 40848 & 36964 & 52952 & 1889 & 2092 \\
\hline
\end{tabular}

\section{A.4 Miscellaneous}

DVI: ideal vs. $\mathrm{C}++$. As noticed earlier, the devirtualization scheme (DVI) proposed here differs from $\mathrm{C}++$ specification: Table XIV compares DVI with the best hand-made $\mathrm{C}++$ implementation, with the same sound MI semantics.

Time efficiency. Assessing time efficiency from those spacial benchmarks requires quite hypothetical assumptions. The time overhead is mostly in the shifts, either static or dynamic. Thus the subobjects number is a good indication of null shifts (Table VII), whereas upcast tables size is a good indication of the number of shifts that are statically avoided (Table III). For method calls, one can compare thunks with the other technique: if one assumes that all table entries are equiprobable, the proportion of null shifts in method calls is exactly the ratio of SST table size $(s)$ and the size of tables in the considered technique. Therefore, with ESO, thunks save on between $23 \%$ and $40 \%$ of all shifts in method calls, whereas, with DVI from $50 \%$ to $100 \%$ are saved.

\section{B. PSEUDO-CODE SURVEY}

The processor specifications are mostly the same as in Driesen [1995; 1999]: particularly, 2 load instructions cannot be executed in parallel, but need a one cycle delay.

\section{B.1 Single subtyping}

Method call:

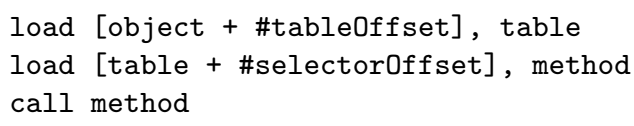

Tableoffset is a constant for all types and methods whereas selectorOffset depends on the method.

Attribute access:

load [object + \#attributeOffset], attribute

AttributeOffset depends on the attribute.

Type check using Schubert's numbering:

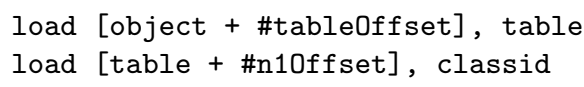

$\overline{21}$ One assumes here that code instructions and addresses have the same size: this may be not the case on 64 -bit processors.

RR LIRMM 2002-174 


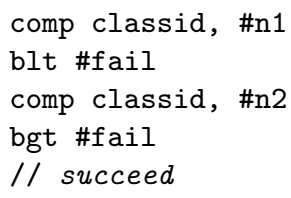

$\mathrm{N} 10 \mathrm{f}$ set is a constant, whereas $\mathrm{n} 1$ and $\mathrm{n} 2$ depend on the target class. Fail is the address of the code which signals an exception, either shared by several or even all type checks, or proper to each one.

Type check using coloring:

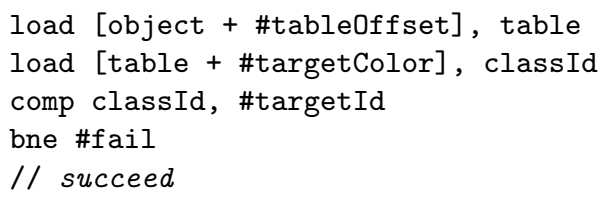

TargetColor and TargetId depend on the target class.

\section{B.2 Multiple inheritance}

Method call:

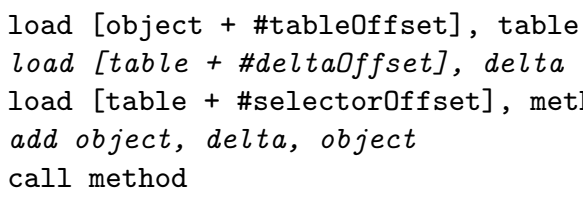

DeltaOffset and selectorOffset depend on both the method and the receiver's static type.

Thunks:

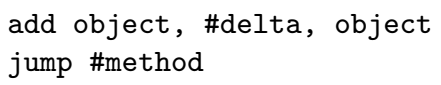

Delta and method depend on both the method and the receiver's static type. When method table points to the thunk, the code is that of single subtyping, whereas, when the thunk is inlined in the method table, it changes to:

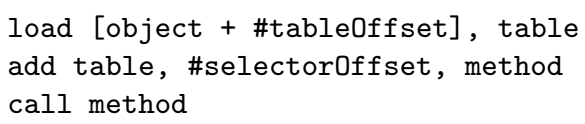

Equality test, with unrelated types:

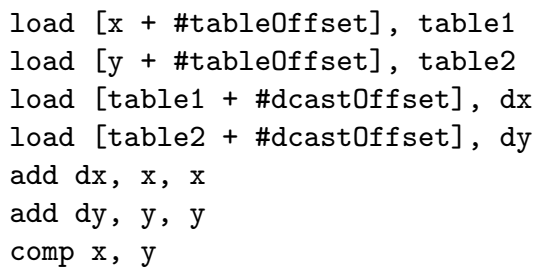

Dcastoffset is a constant. When types are related, this simplifies to:

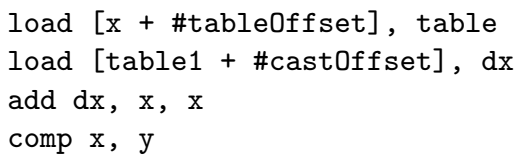

and castoffset depends on both types.

Upcast:

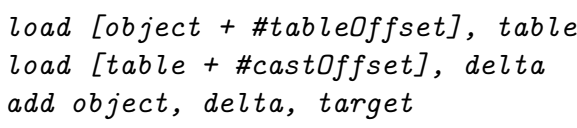


Cast0ffset depends on the static types of both the source and the target.

Accesses to attributes

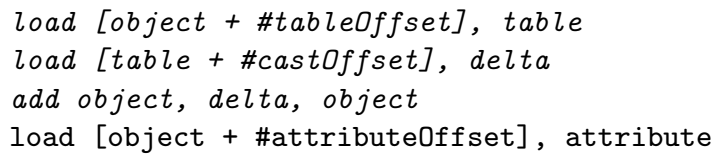

Castoffset depends on both the receiver's static type $\left(\tau_{s}\right)$ and on the class introducing the attribute $\left(T_{p}\right)$ whereas attribute0ffset is an attribute invariant $\left(\delta_{p}\right)$.

Assignment $a . x:=b . y$ :

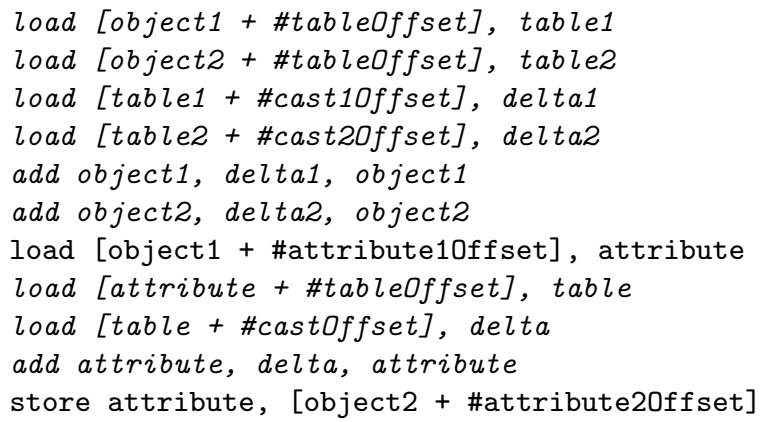

\section{B.3 Alternatives}

NVI code for method call is exactly the same as for MI, whereas NVI code for attribute access is the same as for SST: indeed, the required shift is static, so in the SST code, attributeOffset is augmented by the value of the shift.

Method call with shared tables:

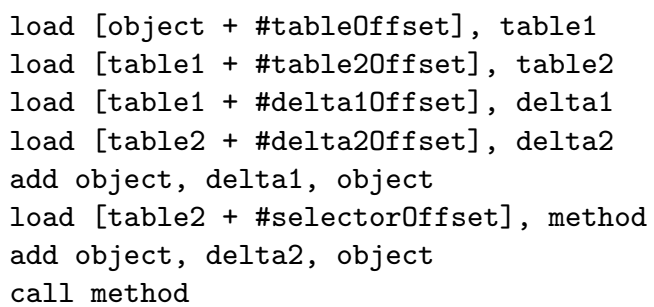

Upcasts with i-VBPTRs:

load [object + \#castOffset], object

or with e-VBPTRs:

load [object + \#castOffset], object

add object, \#delta, object

and attribute access with VBPTRS (either i-VBPTRs or e-VBPTRs):

load [object + \#castoffset], object

load [object + \#attributeOffset], attribute

In both cases, e-VBPTRs are considered in the case of ideal devirtualization: delta is the relative position of the target class in a non virtual part and attributeOffset includes this delta.

Simulating accessors to attributes is exactly as with standard MI:

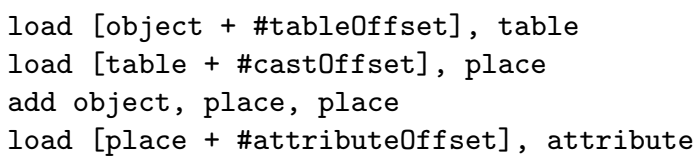

Finally, inline cache of method tables reduces method calls to: 
load [tableObject + \#selectorOffset], method

$L+B$

call method

whereas upcasts are exactly as with VBPTRs, but tableObject replaces object:

load [tableObject + \#castOffset], tableObject

and attributes are accessed through accessors.

\section{B.4 Complements}

B.4.1 Covariant overriding. In single subtyping, for attributes, with Shubert's numbering:

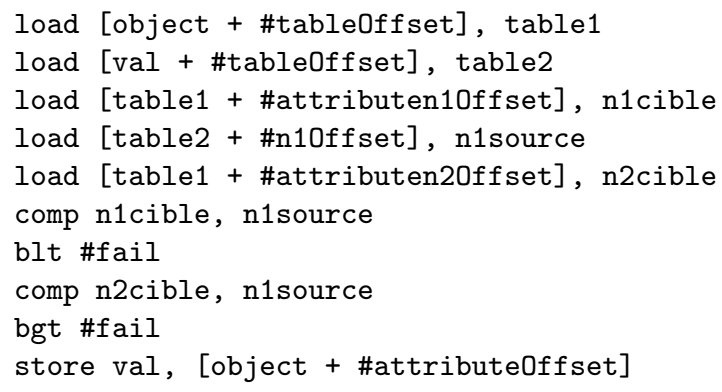

Covariant return type in multiple inheritance, in a thunk:

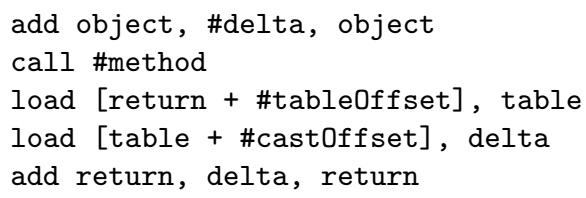

B.4.2 Genericity. Method call to formal type with offset conversion:

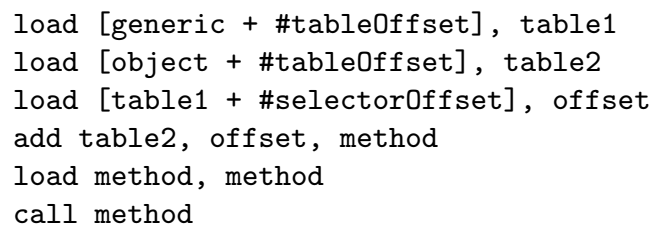

Generic is the current receiver, instance of a parameterized class, and object is an object typed by the formal type.

Attributes in multiple inheritance:

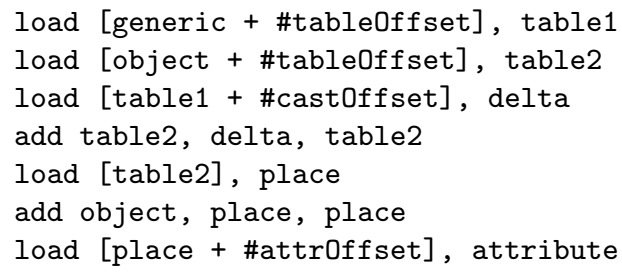

\section{B.5 Global techniques}

B.5.1 Type prediction. When $n$ types are predicted, with ordered identifiers $t_{1}, \ldots, t_{n}$, the code for method call will be a balanced binary tree:

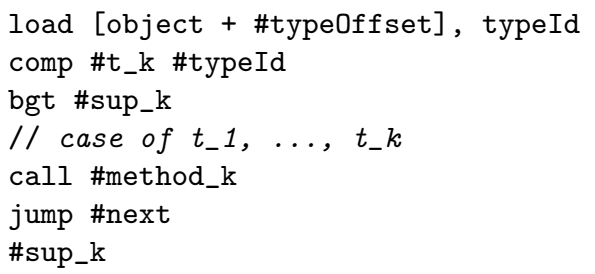




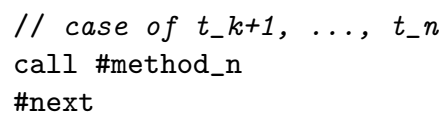

When $k=1$ (resp. $n-1$ ), there is a static call to \#method_1 (resp. \#method_n). The dichotomy depends on the choice of $k$. The ordered list $\left\{t_{1}, \ldots, t_{n}\right\}$ may be partioned into segments of equal method values: the best is to choose $k$ as an extremity of the median segment. When such a $k$ does not exist, i.e. when all values are equal, method call is static. If one assumes a uniform probability $p$ of right prediction for all conditional branches and that method_k is different of method_k +1 , for all $k$, then the average time of method call will be $L+(1+p+B(1-p)) \log _{2}(n)$.

The case of attributes is the same.

B.5.2 Coloring. Coloring does not need a specific pseudo-code: it uses exactly that of single subtyping, except for attributes when the code for simulating accessors is used instead. 